\title{
Extracellular matrix remodeling and vascular complications in type1 diabetes
}

Citation for published version (APA):

Peeters, S. A. (2017). Extracellular matrix remodeling and vascular complications in type1 diabetes. [Doctoral Thesis, Maastricht University]. Datawyse / Universitaire Pers Maastricht. https://doi.org/10.26481/dis.20171220sp

Document status and date:

Published: 01/01/2017

DOI:

10.26481/dis.20171220sp

Document Version:

Publisher's PDF, also known as Version of record

\section{Please check the document version of this publication:}

- A submitted manuscript is the version of the article upon submission and before peer-review. There can be important differences between the submitted version and the official published version of record.

People interested in the research are advised to contact the author for the final version of the publication, or visit the DOI to the publisher's website.

- The final author version and the galley proof are versions of the publication after peer review.

- The final published version features the final layout of the paper including the volume, issue and page numbers.

Link to publication

\footnotetext{
General rights rights.

- You may freely distribute the URL identifying the publication in the public portal. please follow below link for the End User Agreement:

www.umlib.nl/taverne-license

Take down policy

If you believe that this document breaches copyright please contact us at:

repository@maastrichtuniversity.nl

providing details and we will investigate your claim.
}

Copyright and moral rights for the publications made accessible in the public portal are retained by the authors and/or other copyright owners and it is a condition of accessing publications that users recognise and abide by the legal requirements associated with these

- Users may download and print one copy of any publication from the public portal for the purpose of private study or research.

- You may not further distribute the material or use it for any profit-making activity or commercial gain

If the publication is distributed under the terms of Article $25 \mathrm{fa}$ of the Dutch Copyright Act, indicated by the "Taverne" license above, 
Extracellular matrix remodeling and vascular complications in type 1 diabetes 
Copyright (C) Stijn Peeters, Maastricht 2017

No part of this book may be reproduced or transmitted in any form or by any means, without prior permission in writing by the author, or when appropriate, by the publishers of the publications.

Cover Design: Jean Scheijen | vierdrie.nl

Layout: Tiny Wouters

Published by: Datawyse | Universitaire Pers Maastricht

ISBN: 9789461597588

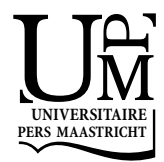




\title{
Extracellular matrix remodeling and vascular complications in type 1 diabetes
}

\author{
PROEFSCHRIFT \\ ter verkrijging van de graad van doctor aan de Universiteit Maastricht, \\ op gezag van de Rector Magnificus, Prof. dr. Rianne M. Letschert, \\ volgens het besluit van het College van Decanen, \\ in het openbaar te verdedigen op \\ woensdag 20 december 2017 om 14.00 uur \\ door
}

Stijn Armand Peeters 


\section{Promotores}

Prof. dr. C.D.A. Stehouwer

Prof. dr. C.G. Schalkwijk

\section{Copromotores}

Dr. L. Engelen

Dr. J. Buijs, Zuyderland Ziekenhuis Heerlen

\section{Beoordelingscommissie}

Prof. dr. N.C. Schaper (voorzitter)

Dr. ir. K.D. Reesink

Prof. dr. A.J. Smit, UMC Groningen

Dr. S.S. Soedamah-Muthu, WUR, Wageningen

Prof. dr. ir. M.P. Weijenberg

Financial support by the Dutch Heart Foundation for the publication of this thesis is gratefully acknowledged 


\section{Contents}

Chapter 1 General introduction

Chapter 2 Serum levels of matrix metalloproteinase-2, -3, -10, and tissue

inhibitor of metalloproteinase-1 are associated with vascular complications in patients with type 1 diabetes.

The EURODIAB Prospective Complications Study

Chapter 3 Plasma matrix metalloproteinases are associated with incident cardiovascular disease and all-cause mortality in patients with type 1 diabetes: a 12-yr follow-up study

Chapter 4 Circulating matrix metalloproteinases are associated with arterial stiffness in patients with type 1 diabetes: pooled analysis of three cohort studies

Chapter 5 Associations between advanced glycation endproducts and matrix metalloproteinases and its inhibitor in individuals with type 1 diabetes

Chapter 6 Summary and general discussion

Nederlandse samenvatting

Valorization

Dankwoord

Curriculum vitae

Scientific ouput

Abbreviations 



\section{1}

General introduction 



\section{General introduction}

Diabetes mellitus is characterized by elevated blood glucose levels, i.e. hyperglycemia. Most newly diagnosed diabetic patients have complaints of polyuria, polydipsia and weight loss. Different pathophysiological mechanisms can be involved in causing hyperglycemia. Type 1 diabetes is characterized by an absolute insulin deficiency. Insulin secreting beta-cells ( $\beta$-cells) in the pancreas are degraded following an autoimmune response against these cells. In contrast to the absolute insulin deficiency in type 1 diabetes, type 2 diabetes is characterized by a relative insulin deficiency, caused by tissue resistance for insulin and increased endogenous glucose production. Excess insulin production by the $\beta$-cells cannot fully attenuate the hyperglycemic state. In Latent Autoimmune Diabetes of the Adult (LADA), an alternate form of type 1 diabetes, slower degradation of $\beta$-cell mass is observed compared to type 1 diabetes. Maturity-Onset Diabetes of the Young (MODY) is a form of type 2 diabetes in young patients in which an autosomal dominant inherited gene defect leads to ineffective insulin production and release from $\beta$-cells.

Initial treatment options differ between the various types of diabetes. Treatment of type 1 diabetes consists of insulin administration preventing the most feared complications including diabetic ketoacidosis. In type 2 diabetes, oral blood glucose lowering medication is the first treatment option, whereas initial insulin treatment is rarely necessary in newly diagnosed type 2 diabetes.

In 2015, about 415 million diabetic patients are known worldwide and the total number will increase to 642 million in $2040 .^{1}$ To date, about one million individuals in the Netherlands are diagnosed ${ }^{1}$ with diabetes mellitus. About $5-10 \%$ of these patients suffer from type 1 diabetes and their number is increasing as well. During the period 1990-1999, the worldwide incidence of type 1 diabetes varied between 0.1 and 40.9 per 100.000 per year, with an annual increase in incidence of $2.8 \%{ }^{2}$ In the period between 1980 and 2005, the incidence of type 1 diabetes in Finnish children under the age of 14 years was doubled in 2005 compared to $1980 .^{3}$

As this thesis focuses on mechanisms leading to complications in type 1 diabetes, only this form of diabetes will further be introduced.

\section{Type 1 diabetes mellitus}

Type 1 diabetes related $\beta$-cell deficiency is caused by autoimmune related $\beta$-cell destruction, probably triggered by one or more environmental factors. ${ }^{4}$ Blood levels of different autoantibodies can be determined, for example autoantibodies against glutamic acid decarboxylase (GAD65), ${ }^{5}$ insulinoma antigen (IA), ${ }^{6}$ islet cells (IC) ${ }^{4}$ and insulin. ${ }^{7}$ In $85-90 \%$ of the patients autoantibodies can be detected at diagnosis. Initial $\beta$-cell mass decrease will not result in hyperglycemia, since the remaining $\beta$-cells can 
produce enough insulin to compensate this decrease. In a later stage, the remaining $\beta$-cells can no longer produce enough insulin to maintain normal blood glucose levels. ${ }^{8}$ Hyperglycemia causes increased renal glucose excretion causing osmotic diuresis, resulting in polyuria, thirst and weight loss. ${ }^{9}$ Ketoacidosis is a severe acute complication, associated with a substantial mortality-rate, and is caused by the presence of increased amounts of acidic ketone bodies, caused by lipolysis in cells deprived of glucose as energy source. ${ }^{10}$ Treatment consists of insulin treatment to lower blood glucose levels and prevent acidemia. ${ }^{10}$

\section{Vascular complications in type 1 diabetes}

Type 1 diabetes is associated with a more than twofold increased risk of cardiovascular disease (CVD), ${ }^{11}$ which is mainly caused by coronary heart disease. ${ }^{11}$ In addition, the risk of cerebrovascular or peripheral artery disease is also significantly increased. ${ }^{11}$ Moreover, these events occur at a younger age compared to the non-diabetic population. $^{11}$

Microvascular complications consist of renal and/or retinal vascular pathology. Albuminuria refers to excess renal albumin loss. Microalbuminuria is defined as urinary albumin excretion (UAE) rates between 20 and 200 microgram per minute $(\mu \mathrm{g} / \mathrm{min}$ ) or 30 and 300 milligrams per 24 hours. In macroalbuminuric state renal albumin loss exceeds $200 \mu \mathrm{g} / \mathrm{min}$ or $300 \mathrm{mg} / 24 \mathrm{~h}{ }^{12}$ The prevalence of microalbuminuria in type 1 diabetic patients increases after onset of the disease, with a prevalence of $33 \%$ within the first 20 years of diabetes, ${ }^{13}$ further increasing to about $50 \%$ after 30 years. ${ }^{14}$ Moreover, microalbuminuria is strongly associated with CVD. ${ }^{15,16}$ Initial renal histological glomerular pathology consists of increased mesangial cell expansion, glomerular basement membrane thickening and glomerulosclerosis. ${ }^{17}$

Retinopathy is characterized by increased retinal vascular occlusion and permeability, leading to macular edema, microaneurysms, lipid exudates and microhemorrhages. ${ }^{18}$ These findings are referred to as non-proliferative retinopathy. Enhancement of these features and presence of neovascularisation and hemorrhages are classified as proliferative retinopathy. ${ }^{18}$ The annual incidence of any visual impairment in type 1 diabetic patients decreased by $76 \%$ (to $0.28 \%$ ) during a 25 -year follow-up study. ${ }^{19}$ A decrease of $61 \%$ (to $12.5 \%$ ) in cumulative incidence of proliferative retinopathy was observed in another prospective observational study with a duration of more than 20 years. ${ }^{20}$ An 18-year follow-up study also showed a decreased cumulative incidence of diabetic retinopathy to $39 \%{ }^{21}$ These decreases in retinopathy were attributable to improved glycemic control, antihypertensive treatment and reduced prevalence of smoking. Intensive glucose treatment ${ }^{22}$ and antihypertensive treatment with reninangiotensin-aldosterone system inhibitors also reduces urinary albumin excretion in 
patients with microalbuminuria. Moreover, a delayed progression to macroalbuminuria is also proven. ${ }^{23}$ As shown by the DCCT/EDIC trial ${ }^{24}$ and summarized in a recent review, ${ }^{25}$ intensive glucose lowering treatment significantly decreases the risk for macro- as well as microvascular complications.

Several contributing factors to the increased risk of vascular complications are known including hyperglycemia, ${ }^{26}$ hypoglycemia, ${ }^{27}$ obesity and insulin resistance, ${ }^{28-30}$ hypertension, $^{31}$ dyslipidemia, ${ }^{32}$ smoking, $^{33}$ oxidative stress, $^{34}$ inflammation $^{35}$ and endothelial dysfunction. ${ }^{36-38}$

\section{Vascular disease and extracellular matrix remodeling}

The full mechanism leading to macro- and microvascular complications in type 1 diabetes has not been fully elucidated. This thesis focuses on the contribution of extracellular matrix (ECM) remodeling in these vascular complications. The ECM provides biochemical and structural support between cells as well as between cells and the surrounding tissue. ${ }^{39}$ The main components of the ECM are proteoglycans, collagen, elastin, fibronectin and laminin, ${ }^{40}$ which are produced by fibroblasts. ECM remodeling is a process that occurs under normal circumstances; it is highly involved in growth, angiogenesis, wound healing and pregnancy. Additionally, in diabetic patients, histopathological alterations can occur and may lead to vascular complications. Besides the vascular complications mentioned previously, arterial stiffness is higher in individuals with type 1 diabetes compared to non-diabetic controls ${ }^{41}$ and has been shown to be associated with CVD in type I diabetic patients. ${ }^{41-44}$ This could be explained by different mechanisms leading to increased accumulation or altered composition of the ECM. ${ }^{45-49}$

\section{Matrix metalloproteinases}

ECM remodeling is, apart from fibroblast related matrix component production, dependent on matrix metalloproteinases (MMPs) and other proteinases that degrade the different components of the existing ECM. ${ }^{50,51}$ Thus far twenty-four MMPs are known and all of them have different substrate specificities. Initially, they were classified by structure and substrate specificity into 6 different groups: collagenases (MMP-1, -8 and -13), gelatinases (MMP-2 and -9), stromelysins (MMP-3 and -10), matrilysins (MMP7 and -12), membrane-type metalloproteinases (MMP-14, -15, -16 and -17) and others. Over time, substrates have been observed to overlap between the different categories. An overview of the MMPs studied in this thesis and their substrates is shown in Table 1.1. 
The general protein structure of MMPs is shown in Figure 1.1. MMPs consist of three main parts: the N-terminal propeptide, the C-terminal (hemopexin-like) domain and the catalytic domain. ${ }^{52-54}$ Cystein is the most important amino acid in the propeptide domain and is connected with the $\mathrm{Zn}^{2+}$-ions in the catalytic domain. Removal of this connection activates the proMMP. The C-terminal domain is necessary for protein-protein interactions, e.g. with matrix components or with its inhibitor, tissue inhibitor of metalloproteinase (TIMP). The catalytic domain contains two $\mathrm{Zn}^{2+}$-ions and two or three $\mathrm{Ca}^{2+}$-ions. The first $\mathrm{Zn}^{2+}$-ion is involved in the catalytic function, while $\mathrm{Ca}^{2+}$-ions are necessary for stabilizing the catalytic domain structure. Besides these domains all MMPs, except MMP-23A and B, contain a signal peptide needed for secretion. Membraneassociated MMPs are anchored through glycosylphosphatidylinositol (GPI) or transmembrane segments I or II (TMI or TMII). In addition, specific binding sites (Figure 1.1: $Y, D$ and $G$ ) characterize the different collagenases and specific fibronectin type II molecules are present in MMP-2 and MMP-9, which improve gelatin and collagen degradation.

Table 1.1 Overview of the MMPs studied in this thesis and their substrates

\begin{tabular}{ll}
\hline & Substrates \\
\hline MMP-1 & Collagen type I, II, III, VII, X \\
(Collagenase-1) & $\begin{array}{l}\text { Aggrecan, casein, gelatin, nidogen, perlecan, proteoglycan link protein, serpins, } \\
\text { tenascin-C and versican } \\
\text { MMP-2 }\end{array}$ \\
Collagen type I, IV, V, VII, X, XI, XIV \\
Aggrecan, elastin, fibronectin, gelatin, laminin, nidogen, proteoglycan link protein \\
and versican \\
Collagen type II, IV, IX, X \\
(Stromelysin-1) & Aggrecan, casein, decorin, elastin, fibronectin, gelatin, laminin, nidogen, perlecan, \\
& proteoglycan link protein and versican \\
MMP-9 & Collagen type IV, V, VII, X, XIV \\
(Gelatinase-B) & Fibronectin, laminin, nidogen, proteoglycan link protein and versican \\
MMP-10 & Collagen type III, IV, V \\
(Stromelysin-2) & Gelatin, fibronectin, laminin and nidogen \\
\hline
\end{tabular}

A variety of cells are able to produce MMPs, for example: monocytes, macrophages, epithelial cells, endothelial cells, osteoclasts, fibroblasts and smooth muscle cells. These various MMP producing cells each have their own specific pattern of MMP production.

MMP production can be activated by G-protein-coupled receptor agonists (e.g. angiotensine II and catecholamines), pro-inflammatory cytokines, growth factors, proteinases and cellular stress. ${ }^{55}$ Regulation of MMP gene expression occurs through various intracellular pathways including activating protein-1 (AP-1) and -2 (AP-2), nuclear factor-KB (NF-KB), Janus Kinase - Signal Transducer and Activator of Transcription (JAK-STAT), SMAD, extracellular signal-regulated kinase/mitogen-activated protein kinase (ERK/MAPK) and c-Jun N-terminal kinases (JNK). ${ }^{53,55}$ Subsequently, MMPs are secreted as non-active enzymes (proMMPs or zymogens) or as membrane-anchored 
types. MMPs need to be activated by proteases, including other MMPs, or chemical agents, before they can perform their matrix degrading activity. Activation of MMPs occurs through cleavage of the propeptide by intra- and intermolecular processes. Non-proteolytic activation occurs through chemical modification of the cysteine-switch. Additional intra- and intermolecular cleavage of the propeptide leads to full activation. $^{50,53}$ The main activating step is removing the cysteine switch, exposing the active site of the enzyme, the catalytic $\mathrm{Zn}^{2+}$-domain.

\section{Archetypal MMPs}

Collagenases

Collagenase-1 (MMP-1)

Collagenase-2 (MMP-8)

Collagenase-3 (MMP-13)

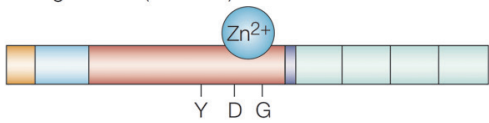

Stromelysins

Stromelysin-1 (MMP-3)

Stromelysin-2 (MMP-10)

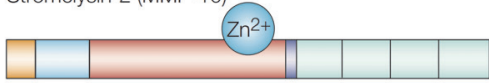

Other MMPs

Metalloelastase (MMP-12)

MMP-19

Enamelysin (MMP-20

MMP-27 (MMP-22, C-MMP)

$\mathrm{Zn}{ }^{2}$

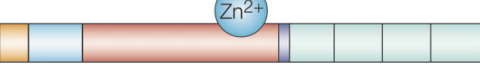

\section{Matrilysins}

Matrilysin (MMP-7) Matrilysin-2 (MMP-26)

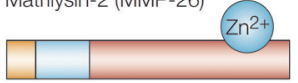

\section{Gelatinases}

Gelatinase-A (MMP-2)

Gelatinase-B (MMP-9)

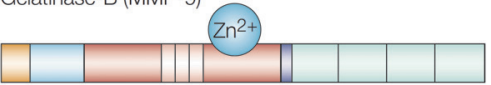

\section{Convertase-activatable MMPs}

\section{Secreted}

Stromelysin-3 (MMP-11)

MMP-21 (X-MMP)

Epilysin (MMP-28)

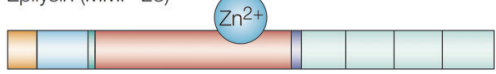

Membrane-associated

MT1-MMP (MMP-14)

MT2-MMP (MMP-15)

MT3-MMP (MMP-16)

MT5-MMP (MMP-24)

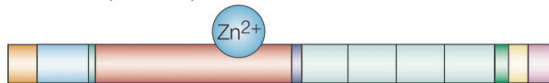

MT4-MMP (MMP-17) MT6-MMP (MMP-25)
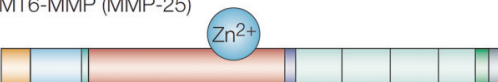

MMP-23A

MMP-23B

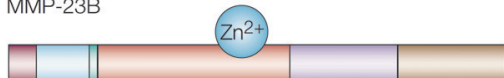

\begin{tabular}{|lll}
\hline Signal peptide & $\square$ Catalytic domain & $\square$ CA \\
$\square$ Propeptide & $\square$ Hinge/linker & $\square$ Cytoplasmic tail \\
Convertase cleavage site & $\square$ Ig-like \\
$\square$ Fibronectin type II modules & $\square$ Membrane linker & $\square$ GPI
\end{tabular}

Figure 1.1 Overview of the general protein structures of the different MMP classes, adapted from ${ }^{52}$ 


\section{Matrix metalloproteinase inhibition}

ECM remodeling is a tightly regulated process. Continuous MMP activity could lead to structurally altered ECM that potentially does not support tissues as intended. Therefore, MMPs have their own inhibitors, TIMPs. ${ }^{56,57}$ An overview of TIMPs and their specific MMP substrates is shown in Table 1.2. TIMPs regulate cell functions through MMP-dependent as well as MMP-independent mechanisms, including cell proliferation, apoptosis and differentiation. ${ }^{58}$ TIMPs form a non-covalent 1:1 complex with MMPs and specific N-terminal domains on the TIMP molecule inhibit MMP activity. ${ }^{58}$

Alfa-2-macroglobulin, tissue factor pathway inhibitor-2, doxycycline, simvastin and bisphosphonates are other known MMP inhibitors. ${ }^{51}$ Furthermore, specific MMP inhibitors have been designed. ${ }^{54}$ Overall, the proteolytic activity of the MMPs is controlled at three known levels: transcription, activation of the zymogens, and inhibition of the active forms by various TIMPs or other inhibitors.

Table 1.2 Overview of tissue inhibitors of metalloproteinases (TIMPs) and their substrates

\begin{tabular}{|c|c|c|c|c|}
\hline & TIMP-1 & TIMP-2 & TIMP-3 & TIMP-4 \\
\hline MMP inhibition & $\begin{array}{l}\text { All, but weak for } \\
\text { MMP-14, -16, -19 } \\
\text { and }-24\end{array}$ & All & All & Most \\
\hline proMMP inhibition & proMMP-9 & proMMP-2 & $\begin{array}{l}\text { proMMP-2 and } \\
\text { proMMP-9 }\end{array}$ & proMMP-2 \\
\hline Localisation & $\begin{array}{l}\text { Soluble and cell } \\
\text { surface }\end{array}$ & $\begin{array}{l}\text { Soluble and cell } \\
\text { surface }\end{array}$ & $\begin{array}{l}\text { Extracellular matrix } \\
\text { and cell surface }\end{array}$ & $\begin{array}{l}\text { Soluble and cell } \\
\text { surface }\end{array}$ \\
\hline
\end{tabular}

\section{Type 1 diabetes, extracellular matrix remodeling and vascular complications}

In diabetes, several mechanisms contribute to ECM remodeling with and without increased vascular fibrosis. Hyperglycemia, proinflammatory cytokines and growth factors (e.g. transforming growth factor- $\beta$ (TGF- $\beta$ ) and connective tissue growth factor (CTGF)) are stimulators of fibroblast-related ECM production. Matrix accumulation leads to altered ECM composition and function. Most stimulators of matrix accumulation are also known to increase MMP production. ${ }^{59,60}$ Possibly in early phase of diabetes, upregulation of MMPs at tissue level can be observed, eventually leading to suppression of the MMP system and increased matrix deposition, leading to increased fibrosis. ${ }^{61}$ This can be accompanied by an increase of MMP inhibitors or TIMPs. In addition, increased MMP production could accompany the fibroblast mediated ECM production or is increased by previously mentioned stimulators. Growth factors, like TGF- $\beta$, are anchored 
in the ECM and MMP-mediated ECM degradation related TGF- $\beta$ release can lead to fibroblast stimulation and increase ECM production. ${ }^{62}$

An overview of possible pathways leading to changes in the ECM is shown in Figure 1.2.

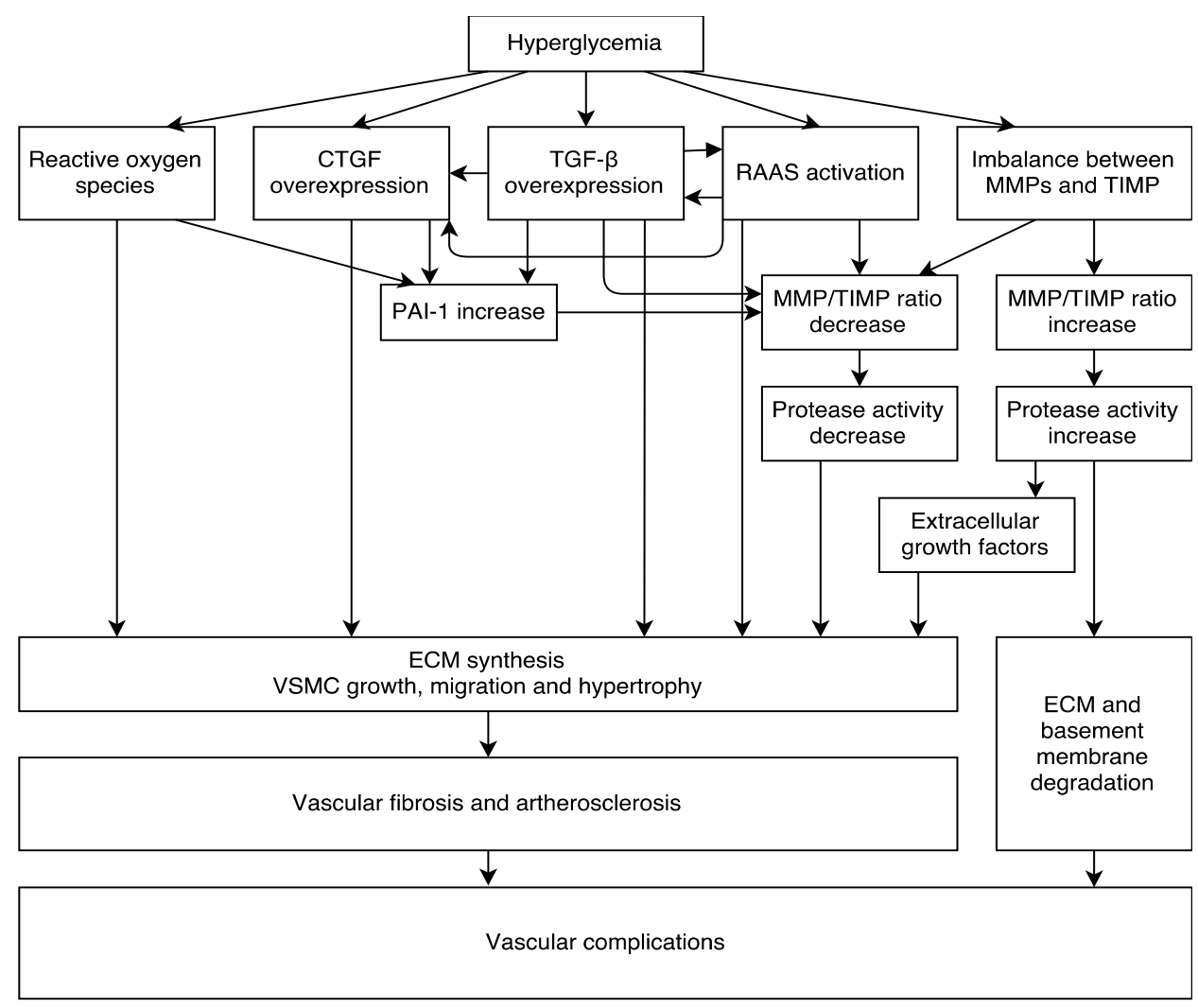

Figure 1.2 Pathways leading to altered ECM remodeling and vascular complications

CTGF, connective tissue growth factor; TGF- $\beta$, transforming growth factor- $\beta$; RAAS, renin angiotensin aldosterone system; PAI-1, plasminogen activator inhibitor-1; MMP, matrix metalloproteinase; TIMP, tissue inhibitor of metalloproteinase; ECM, extracellular matrix; VSMC, vascular smooth muscle cell

Most studies regarding ECM remodeling by MMPs at tissue level in type 1 diabetes were performed in type 1 diabetic animal models and investigated cardiovascular complications, ${ }^{63,64}$ renal changes with ${ }^{65-69}$ and without the presence of albuminuria ${ }^{70-72}$ and retinopathy. ${ }^{73-77}$

Only two studies investigated changes at renal tissue level in humans. In patients with type 1 diabetes $^{78}$ changes were observed in glomerular MMP-2, MMP-3 and type IV 
collagen mRNA expression, but an evident pattern linked to ECM accumulation was not observed. In a study in type 2 diabetic patients, ${ }^{79}$ glomerular MMP-3, TIMP-1 and type IV collagen mRNA expression was inversely associated with mesangial cell expansion, a marker of diabetic kidney disease. However, interstitial renal injury was positively associated with MMP-3, TIMP-1 and type IV collagen mRNA expression. In addition, in retinal tissues of type 1 diabetic patients with proliferative retinopathy higher levels of MMP-2, MMP-3, MMP-9 and TIMP-1 were observed compared to non-diabetic controls. $^{80}$

However, in clinical practice it is hardly possible to evaluate the macro- and microvascular ECM status at tissue level. Therefore, determination of circulating MMPs and/or TIMPs could be a substitute or surrogate marker for the effect at tissue level.

In type 1 diabetic patients, circulating levels of MMP- $2{ }^{81,82} \mathrm{MMP}-9^{82}$ and MMP-10 ${ }^{83}$ have been observed to be higher compared to non-diabetic controls. Associations between circulating levels of MMPs or TIMPs and macrovascular complications in type 1 diabetic patients have not been published. However, associations between microvascular complications and circulating levels of MMPs and TIMP-1 have been investigated, but literature is scarce. Significant associations between serum MMP-9 and mild to moderate diabetic retinopathy ${ }^{84}$ as well as associations between plasma MMP10 and diabetic nephropathy and proliferative retinopathy ${ }^{83}$ have been observed.

Most studies were relatively small and focussed on only one or two MMPs or TIMPs. They were mostly aimed at associations with MMP-2 and MMP-9, since both are known to degrade basement membrane related type IV collagen.

\section{Advanced Glycation Endproducts}

Besides the potential contribution of the MMP-TIMP system to vascular complications, advanced glycation endproducts (AGEs) have been associated with vascular complications in type 1 diabetic patients. ${ }^{85}$ Furthermore, in vitro studies showed associations between AGEs and markers of the MMP-TIMP system. ${ }^{86}$

The formation of AGEs occurs through various steps. Maillard was the first to describe the reaction between the carbonylgroup of sugars with protein residues. ${ }^{87}$ This reaction leads to the formation of a Schiff base, resulting in Amadori products, of which glycated haemoglobin $\mathrm{Alc}(\mathrm{HbA1c})$ is one the best known examples. ${ }^{88}$ These first steps are reversible. Only part of these Amadori products is modified to irreversible AGEs. This slow process can occur within weeks to months and pentosidine is a well-known AGE resulting from this slow reaction. In addition to reduced sugars, intermediates in the glycolysis and lipid peroxidation pathways also contribute to the formation of AGEs. ${ }^{89}$

Glycolytic intermediates have shown to be more potent glycating agents compared to glucose. Methylglyoxal (MGO) is a highly reactive dicarbonyl compound formed during glycolysis. It reacts with arginine residues of proteins to form 5-hydro-5- 
methylimidazolone (MG-H1); i.e. a major AGE. Reaction of MGO with lysine residues leads to the formation of another AGE: $N^{\varepsilon}$-(carboxyethyl)lysine (CEL). In addition, other highly active intermediates, e.g. glyoxal and 3-deoxyglucosone, are also formed during glycolysis and lipid peroxidation. Reaction of these intermediates with lysine residues leads to the formation of $\mathrm{N}^{\varepsilon}$-(carboxymethyl)lysine $(\mathrm{CML}){ }^{90}$

AGEs can interfere with cellular function through three different pathways. ${ }^{90}$ Firstly, they have the ability to form crosslinks, leading e.g. to increased vascular stiffness. Secondly, cellular functions can be altered by the intracellular formed AGEs. Thirdly, activation of the receptors of AGE by AGEs results in activation of intracellular pathways leading to altered gene expression. Various AGEs have been previously associated with vascular complications in type 1 diabetes. ${ }^{85,91,92}$

\section{Possible mediation in the associations between markers of the MMP-TIMP system and vascular complications}

\section{Renal dysfunction}

Markers of renal dysfunction include a decrease in estimated glomerular filtration rate (eGFR) and/or a degree of albuminuria. In the general population, but also in type 1 diabetic patients, ${ }^{93}$ both decreased GFR and albuminuria have been associated with an increased risk of CVD. ${ }^{94,95}$ Involvement of MMPs and TIMP-1 in renal tissue remodeling has been observed in type 1 diabetic animal studies, as mentioned previously. Human studies regarding tissue remodeling have also been performed in type 1 and type 2 diabetic patients, as also mentioned previously. We hypothesize that increased renal tissue remodeling, caused by increased MMPs or TIMP-1 levels, leads to an increased risk of vascular disease (Figure 1.3).

\section{Inflammation}

In atherosclerosis, inflammation is considered to be the main pathological process, ${ }^{96}$ leading to accumulation of immune cells and lipids in the early stage.

In type 1 diabetes, metabolic changes can lead to increased circulating markers of inflammation (C-reactive protein, interleukin-1, interleukin-6, tumor necrosis factor- $\alpha$, fibrinogen, soluble intercellular adhesion molecule-1 and secreted phospholipase A2) and these have already been associated with macro- and microvascular complications. ${ }^{97-100}$ MMPs can be upregulated through non-inflammatory factors, e.g. stretching of vascular smooth muscle cells, and can potentially (further) increase the inflammatory state. ${ }^{101,102}$ However, MMPs are also known to be upregulated through pro-inflammatory cytokines. ${ }^{102}$ MMP related matrix remodeling and tissue injury can lead to increased vascular smooth muscle cell migration to the vascular lumen, monocyte/macrophage infiltration and increased fibrosis, ${ }^{55}$ potentially contributing to an 
increased risk of CVD. Besides possible mediation through renal dysfunction, MMPs may increase inflammation by its actions and further potentiate the risk of vascular disease.

\section{Endothelial dysfunction}

The vascular endothelium and its basement membrane form the continuous intimal layer of the blood vessel and separate the arterial lumen from the ECM. Normal endothelial function consists of regulation of vascular tone and permeability, prevention of adhesion of leukocytes and platelets as well as prevention of local coagulation. ${ }^{103}$ Endothelial dysfunction (ED) is referred to as a change in these properties and could thus lead to increased arterial stiffness, increased permeability and decreased anticoagulant activity. ${ }^{104}$ Circulating markers of ED, e.g. soluble E-selectin, soluble vascular cell adhesion molecule (VCAM) and soluble intercellular adhesion molecule (ICAM), can be determined. $^{104}$

In type 1 diabetic patients, these markers have been independently associated with macro- as well as microvascular disease. ${ }^{38,100,105,106}$ MMP-2 and MMP-9 can cleave vascular tight junctions and basement membrane proteins, leading to decreased vascular integrity and increased vascular permeability. ${ }^{107}$ Therefore, MMPs could increase ED; possibly further increasing the risk of vascular disease.

\section{Aim of this thesis}

The aim of this thesis was to investigate associations between circulating levels of MMP$1,-2,-3,-9$ and -10 , and TIMP-1 and vascular complications in type 1 diabetic patients. For these analyses several studies with different study designs were used. The results presented in this dissertation are based on data from three different studies: EURODIAB, LEACE and PROFIL.

The EURODIAB IDDM Complications study was performed in 3250 type 1 diabetic patients between 1989 and 1991 in 31 centres across Europe. ${ }^{108}$ This study evaluated the prevalence of macro- and microvascular complications as well as other metabolic complications. In 1997, the EURODIAB Prospective Complications Study (PCS) was performed in 1880 patients of the original 3250 patients. ${ }^{109}$

In 1993, 391 type 1 diabetic patients were included in a prospective study at Steno Diabetes Centre, Gentofte, Denmark (LEACE). Diabetic nephropathy was present in 199 patients and was defined as persistent macroalbuminuria (UAE $>300 \mathrm{mg} / 24 \mathrm{~h}$ ) in at least two out of three previous consecutive 24-h urine collections, presence of retinopathy, and absence of other kidney or urinary tract disease. 192 normoalbuminuric type 1 diabetic patients were enrolled as controls. They were matched for sex, age and 
duration of diabetes. During a median follow-up of 12.3 years incident (non-)fatal cardiovascular events as well as all-cause mortality were observed. ${ }^{110,111}$

Between September 2009 and June 2011, 676 type 1 diabetic patients were enrolled in a cross-sectional study (PROFIL) evaluating associations between blood pressure, arterial stiffness and diabetic vascular complications. ${ }^{41,112,113}$ Blood pressure measurements were performed for 24 hours with a validated tonometric device and arterial stiffness was evaluated through carotid-femoral pulse wave velocity (cfPWV). Besides different degrees of albuminuria, also different degrees of retinopathy and autonomic dysfunction were evaluated.

In the first study, as described in chapter two, we have investigated serum levels of the previously mentioned markers in relation to prior CVD, albuminuria status and retinopathy status in the cross-sectional nested case-control study derived from the EURODIAB PCS. ${ }^{114}$ Full case analysis was possible in 1220 patients regarding vascular complications. A cross-sectional nested case-control study $(n=543)^{115}$ was designed to evaluate cases with the greatest complication burden (prior CVD, macroalbuminuria and/or proliferative retinopathy) and patients without complications. We used the nested case-control study to investigate associations between serum levels of previously mentioned MMPs and TIMP-1 and vascular complications (chapter two) and pulse pressure (PP) as marker of arterial stiffness (chapter four).

In the second study, as described in the third chapter, we have investigated the associations between plasma biomarker levels at baseline and incident (non-)fatal CVD and all-cause mortality in the prospective LEACE-study ${ }^{116}$ with a median follow-up of 12.3 years. Analysis was performed in 337 of the original 391 patients in whom all data was present regarding MMPs, TIMP-1, and potential confounders and / or mediators.

The fourth chapter described the associations between circulating MMPs and TIMP-1 and markers of arterial stiffness, cfPWV and PP. CFPWV was present in the PROFIL study and this cross-sectional study ${ }^{41}$ was performed in Denmark and investigated arterial stiffness and diabetic complications in individuals with type 1 diabetes. To investigate the associations regarding markers of the MMP-TIMP system and PP we combined the three different studies: EURODIAB, LEACE and PROFIL.

Finally, in the fifth chapter we have described associations between circulating AGEs and serum levels of the previously mentioned MMPs and TIMP-1 in the PROFIL study.

All these associations were adjusted for established confounders and presence of vascular complications. Possible mediation through markers of renal dysfunction, inflammation and ED was also investigated in chapter two and three (Figure 1.3). 


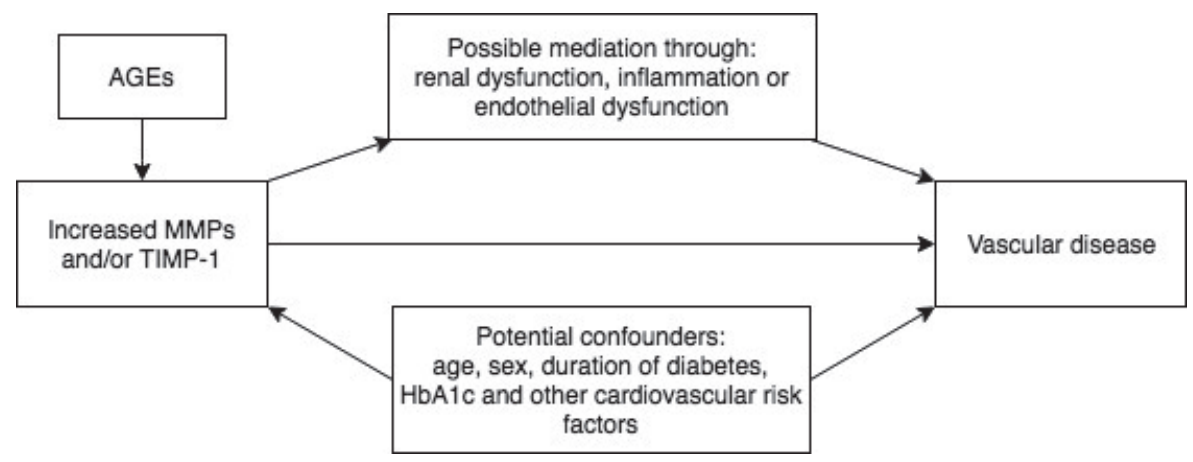

Figure 1.3 Our hypothesis: increased circulating MMPs and/or TIMP-1 associated with vascular disease, with potential confounders and possible mediation routes

AGEs, advanced glycation endproducts; MMP, matrix metalloproteinase; TIMP, tissue inhibitor of metalloproteinase; HbA1c, glycated haemoglobin A1c 


\section{References}

1. IDF Diabetes Altas Seventh Edition, International Diabetes Federation 2015.

2. ProjectGroup D: Incidence and trends of childhood Type 1 diabetes worldwide 1990-1999. Diabet Med 2006;23:857-866

3. Harjutsalo V, Sjoberg L, Tuomilehto J: Time trends in the incidence of type 1 diabetes in Finnish children: a cohort study. Lancet 2008;371:1777-1782

4. Atkinson MA, Maclaren NK: The pathogenesis of insulin-dependent diabetes mellitus. N Engl J Med 1994;331:1428-1436

5. Baekkeskov S, Aanstoot HJ, Christgau S, Reetz A, Solimena M, Cascalho M, Folli F, Richter-Olesen H, De Camilli P: Identification of the 64K autoantigen in insulin-dependent diabetes as the GABA-synthesizing enzyme glutamic acid decarboxylase. Nature 1990;347:151-156

6. Hawa M, Rowe R, Lan MS, Notkins AL, Pozzilli P, Christie MR, Leslie RD: Value of antibodies to islet protein tyrosine phosphatase-like molecule in predicting type 1 diabetes. Diabetes 1997;46:1270-1275

7. Ziegler AG, Hillebrand B, RabI W, Mayrhofer M, Hummel M, Mollenhauer U, Vordemann J, Lenz A, Standl $\mathrm{E}$ : On the appearance of islet associated autoimmunity in offspring of diabetic mothers: a prospective study from birth. Diabetologia 1993;36:402-408

8. Eisenbarth GS: Type I diabetes mellitus. A chronic autoimmune disease. N Engl J Med 1986;314: 1360-1368

9. Brizzolara A, Barbieri MP, Adezati L, Viviani GL: Water distribution in insulin-dependent diabetes mellitus in various states of metabolic control. Eur J Endocrinol 1996;135:609-615

10. Kitabchi AE, Umpierrez GE, Miles JM, Fisher JN: Hyperglycemic crises in adult patients with diabetes. Diabetes Care 2009;32:1335-1343

11. de Ferranti SD, de Boer IH, Fonseca V, Fox CS, Golden SH, Lavie CJ, Magge SN, Marx N, McGuire DK, Orchard TJ, Zinman B, Eckel RH: Type 1 Diabetes Mellitus and Cardiovascular Disease: A Scientific Statement From the American Heart Association and American Diabetes Association. Diabetes Care 2014;37:2843-2863

12. National Kidney Foundation. K/DOQI clinical practice guidelines for chronic kidney disease: evaluation, classification, and stratification. Am J Kidney Dis 2002;39:S1-266

13. Hovind P, Tarnow L, Rossing P, Jensen BR, Graae M, Torp I, Binder C, Parving HH: Predictors for the development of microalbuminuria and macroalbuminuria in patients with type 1 diabetes: inception cohort study. Bmj 2004;328:1105

14. Warram JH, Gearin G, Laffel L, Krolewski AS: Effect of duration of type I diabetes on the prevalence of stages of diabetic nephropathy defined by urinary albumin/creatinine ratio. J Am Soc Nephrol 1996; 7:930-937

15. Jensen T, Borch-Johnsen K, Kofoed-Enevoldsen A, Deckert T: Coronary heart disease in young type 1 (insulin-dependent) diabetic patients with and without diabetic nephropathy: incidence and risk factors. Diabetologia 1987;30:144-148

16. Torffvit O, Lovestam-Adrian M, Agardh E, Agardh CD: Nephropathy, but not retinopathy, is associated with the development of heart disease in Type 1 diabetes: a 12-year observation study of 462 patients. Diabet Med 2005;22:723-729

17. Fioretto P, Steffes MW, Brown DM, Mauer SM: An overview of renal pathology in insulin-dependent diabetes mellitus in relationship to altered glomerular hemodynamics. Am J Kidney Dis 1992;20:549-558

18. Antonetti DA, Klein R, Gardner TW: Diabetic retinopathy. N Engl J Med 2012;366:1227-1239

19. Klein R, Lee KE, Gangnon RE, Klein BE: The 25-year incidence of visual impairment in type 1 diabetes mellitus the wisconsin epidemiologic study of diabetic retinopathy. Ophthalmology 2010;117:63-70

20. Hovind P, Tarnow L, Rossing K, Rossing P, Eising S, Larsen N, Binder C, Parving HH: Decreasing incidence of severe diabetic microangiopathy in type 1 diabetes. Diabetes Care 2003;26:1258-1264

21. Effect of Intensive Diabetes Therapy on the Progression of Diabetic Retinopathy in Patients with Type 1 Diabetes: 18 Years of Follow-up in the DCCT/EDIC. Diabetes 2014; 
22. de Boer IH, Sun W, Gao X, Cleary PA, Lachin JM, Molitch ME, Steffes MW, Zinman B: Effect of intensive diabetes treatment on albuminuria in type 1 diabetes: long-term follow-up of the Diabetes Control and Complications Trial and Epidemiology of Diabetes Interventions and Complications study. Lancet Diabetes Endocrinol 2014;2:793-800

23. Hirst JA, Taylor KS, Stevens RJ, Blacklock CL, Roberts NW, Pugh CW, Farmer AJ: The impact of reninangiotensin-aldosterone system inhibitors on Type 1 and Type 2 diabetic patients with and without early diabetic nephropathy. Kidney Int 2012;81:674-683

24. Nathan DM: The diabetes control and complications trial/epidemiology of diabetes interventions and complications study at 30 years: overview. Diabetes Care 2014;37:9-16

25. Fullerton B, Jeitler K, Seitz M, Horvath K, Berghold A, Siebenhofer A: Intensive glucose control versus conventional glucose control for type 1 diabetes mellitus. Cochrane Database Syst Rev 2014;2:CD009122

26. Larsen J, Brekke M, Sandvik L, Arnesen H, Hanssen KF, Dahl-Jorgensen K: Silent coronary atheromatosis in type 1 diabetic patients and its relation to long-term glycemic control. Diabetes 2002;51:2637-2641

27. Gogitidze Joy N, Hedrington MS, Briscoe VJ, Tate DB, Ertl AC, Davis SN: Effects of acute hypoglycemia on inflammatory and pro-atherothrombotic biomarkers in individuals with type 1 diabetes and healthy individuals. Diabetes Care 2010;33:1529-1535

28. Purnell JQ, Dev RK, Steffes MW, Cleary PA, Palmer JP, Hirsch IB, Hokanson JE, Brunzell JD: Relationship of family history of type 2 diabetes, hypoglycemia, and autoantibodies to weight gain and lipids with intensive and conventional therapy in the Diabetes Control and Complications Trial. Diabetes 2003;52:2623-2629

29. Purnell JQ, Hokanson JE, Marcovina SM, Steffes MW, Cleary PA, Brunzell JD: Effect of excessive weight gain with intensive therapy of type 1 diabetes on lipid levels and blood pressure: results from the DCCT. Diabetes Control and Complications Trial. Jama 1998;280:140-146

30. Erbey JR, Kuller LH, Becker DJ, Orchard TJ: The association between a family history of type 2 diabetes and coronary artery disease in a type 1 diabetes population. Diabetes Care 1998;21:610-614

31. Miller RG, Secrest AM, Ellis D, Becker DJ, Orchard TJ: Changing impact of modifiable risk factors on the incidence of major outcomes of type 1 diabetes: the Pittsburgh Epidemiology of Diabetes Complications Study. Diabetes Care 2013;36:3999-4006

32. Orchard TJ, Forrest KY, Kuller LH, Becker DJ: Lipid and blood pressure treatment goals for type 1 diabetes: 10-year incidence data from the Pittsburgh Epidemiology of Diabetes Complications Study. Diabetes Care 2001;24:1053-1059

33. Eliasson B: Cigarette smoking and diabetes. Prog Cardiovasc Dis 2003;45:405-413

34. Ceriello A: New insights on oxidative stress and diabetic complications may lead to a "causal" antioxidant therapy. Diabetes Care 2003;26:1589-1596

35. Miller RG, Costacou T, Orchard TJ: Lipoprotein-associated phospholipase A2, C-reactive protein, and coronary artery disease in individuals with type 1 diabetes and macroalbuminuria. Diab Vasc Dis Res 2010;7:47-55

36. Nin JW, Ferreira I, Schalkwijk CG, Prins MH, Chaturvedi N, Fuller JH, Stehouwer CD: Levels of soluble receptor for AGE are cross-sectionally associated with cardiovascular disease in type 1 diabetes, and this association is partially mediated by endothelial and renal dysfunction and by low-grade inflammation: the EURODIAB Prospective Complications Study. Diabetologia 2009;52:705-714

37. Chan NN, Vallance P, Colhoun HM: Endothelium-dependent and -independent vascular dysfunction in type 1 diabetes: role of conventional risk factors, sex, and glycemic control. Arterioscler Thromb Vasc Biol 2003;23:1048-1054

38. Costacou T, Lopes-Virella MF, Zgibor JC, Virella G, Otvos J, Walsh M, Orchard TJ: Markers of endothelial dysfunction in the prediction of coronary artery disease in type 1 diabetes. The Pittsburgh Epidemiology of Diabetes Complications Study. J Diabetes Complications 2005;19:183-193

39. Davis GE, Senger DR: Endothelial extracellular matrix: biosynthesis, remodeling, and functions during vascular morphogenesis and neovessel stabilization. Circ Res 2005;97:1093-1107

40. Wagenseil JE, Mecham RP: Vascular extracellular matrix and arterial mechanics. Physiol Rev 2009;89:957-989

41. Theilade S, Lajer M, Persson F, Joergensen C, Rossing P: Arterial stiffness is associated with cardiovascular, renal, retinal, and autonomic disease in type 1 diabetes. Diabetes Care 2013;36:715-721 
42. Stehouwer CD, Henry RM, Ferreira I: Arterial stiffness in diabetes and the metabolic syndrome: a pathway to cardiovascular disease. Diabetologia 2008;51:527-539

43. Schram MT, Chaturvedi N, Fuller JH, Stehouwer CD: Pulse pressure is associated with age and cardiovascular disease in type 1 diabetes: the Eurodiab Prospective Complications Study. J Hypertens 2003;21:2035-2044

44. Theilade S, Lajer M, Jorsal A, Tarnow L, Parving HH, Rossing P: Arterial stiffness and endothelial dysfunction independently and synergistically predict cardiovascular and renal outcome in patients with type 1 diabetes. Diabet Med 2012;29:990-994

45. McNulty M, Mahmud A, Spiers P, Feely J: Collagen type-I degradation is related to arterial stiffness in hypertensive and normotensive subjects. J Hum Hypertens 2006;20:867-873

46. Yasmin, McEniery CM, Wallace S, Dakham Z, Pulsalkar P, Maki-Petaja K, Ashby MJ, Cockcroft JR, Wilkinson IB: Matrix metalloproteinase-9 (MMP-9), MMP-2, and serum elastase activity are associated with systolic hypertension and arterial stiffness. Arterioscler Thromb Vasc Biol 2005;25:372

47. Chatzikyriakou SV, Tziakas DN, Chalikias GK, Stakos DA, Thomaidi AK, Mitrousi K, Lantzouraki AE, Kotsiou S, Maltezos E, Boudoulas H: Serum levels of collagen type-I degradation markers are associated with vascular stiffness in chronic heart failure patients. Eur J Heart Fail 2008;10:1181-1185

48. Wang $\mathrm{M}$, Lakatta EG: Altered regulation of matrix metalloproteinase-2 in aortic remodeling during aging. Hypertension 2002;39:865-873

49. Chung AW, Yang HH, Kim JM, Sigrist MK, Chum E, Gourlay WA, Levin A: Upregulation of matrix metalloproteinase-2 in the arterial vasculature contributes to stiffening and vasomotor dysfunction in patients with chronic kidney disease. Circulation 2009;120:792-801

50. Visse R, Nagase H: Matrix metalloproteinases and tissue inhibitors of metalloproteinases: structure, function, and biochemistry. Circ Res 2003;92:827-839

51. Chistiakov DA, Sobenin IA, Orekhov AN: Vascular extracellular matrix in atherosclerosis. Cardiol Rev 2013;21:270-288

52. Overall CM, Lopez-Otin C: Strategies for MMP inhibition in cancer: innovations for the post-trial era. Nat Rev Cancer 2002;2:657-672

53. Zitka O, Kukacka J, Krizkova S, Huska D, Adam V, Masarik M, Prusa R, Kizek R: Matrix metalloproteinases. Curr Med Chem 2010;17:3751-3768

54. Verma RP, Hansch C: Matrix metalloproteinases (MMPs): chemical-biological functions and (Q)SARs. Bioorg Med Chem 2007;15:2223-2268

55. Berry E, Bosonea AM, Wang X, Fernandez-Patron C: Insights into the activity, differential expression, mutual regulation, and functions of matrix metalloproteinases and a disintegrin and metalloproteinases in hypertension and cardiac disease. J Vasc Res 2013;50:52-68

56. Murphy G: Tissue inhibitors of metalloproteinases. Genome Biol 2011;12:233

57. Brew K, Nagase H: The tissue inhibitors of metalloproteinases (TIMPs): an ancient family with structural and functional diversity. Biochim Biophys Acta 2010;1803:55-71

58. Ries C: Cytokine functions of TIMP-1. Cell Mol Life Sci 2014;71:659-672

59. Ban CR, Twigg SM: Fibrosis in diabetes complications: pathogenic mechanisms and circulating and urinary markers. Vasc Health Risk Manag 2008;4:575-596

60. Lan TH, Huang XQ, Tan HM: Vascular fibrosis in atherosclerosis. Cardiovasc Pathol 2013;22:401-407

61. Portik-Dobos V, Anstadt MP, Hutchinson J, Bannan M, Ergul A: Evidence for a matrix metalloproteinase induction/activation system in arterial vasculature and decreased synthesis and activity in diabetes. Diabetes 2002;51:3063-3068

62. Imai K, Hiramatsu A, Fukushima D, Pierschbacher MD, Okada Y: Degradation of decorin by matrix metalloproteinases: identification of the cleavage sites, kinetic analyses and transforming growth factorbeta1 release. Biochem J 1997;322 ( Pt 3):809-814

63. Eguchi M, Xu G, Li RK, Sweeney G: Diabetes influences cardiac extracellular matrix remodelling after myocardial infarction and subsequent development of cardiac dysfunction. I Cell Mol Med 2012;16:2925-2934

64. Mohammadzadeh F, Desjardins JF, Tsoporis JN, Proteau G, Leong-Poi H, Parker TG: S100B: role in cardiac remodeling and function following myocardial infarction in diabetes. Life Sci 2013;92:639-647 
65. Dixon A, Maric C: 17beta-Estradiol attenuates diabetic kidney disease by regulating extracellular matrix and transforming growth factor-beta protein expression and signaling. Am J Physiol Renal Physiol 2007;293:F1678-1690

66. Mankhey RW, Wells CC, Bhatti F, Maric C: 17beta-Estradiol supplementation reduces tubulointerstitial fibrosis by increasing MMP activity in the diabetic kidney. Am J Physiol Regul Integr Comp Physiol 2007;292:R769-777

67. Takamiya Y, Fukami K, Yamagishi S, Kaida Y, Nakayama Y, Obara N, Iwatani R, Ando R, Koike K, Matsui T, Nishino $\mathrm{Y}$, Ueda S, Cooper ME, Okuda S: Experimental diabetic nephropathy is accelerated in matrix metalloproteinase-2 knockout mice. Nephrol Dial Transplant 2013;28:55-62

68. Maric-Bilkan C, Flynn ER, Chade AR: Microvascular disease precedes the decline in renal function in the streptozotocin-induced diabetic rat. Am J Physiol Renal Physiol 2012;302:F308-315

69. Liu DX, Liu XM, Su Y, Zhang XJ: Renal expression of proto-oncogene Ets-1 on matrix remodeling in experimental diabetic nephropathy. Acta Histochem 2011;113:527-533

70. McLennan SV, Kelly DJ, Cox AJ, Cao Z, Lyons JG, Yue DK, Gilbert RE: Decreased matrix degradation in diabetic nephropathy: effects of ACE inhibition on the expression and activities of matrix metalloproteinases. Diabetologia 2002;45:268-275

71. Sun SZ, Wang Y, Li Q, Tian YJ, Liu MH, Yu YH: Effects of benazepril on renal function and kidney expression of matrix metalloproteinase-2 and tissue inhibitor of metalloproteinase-2 in diabetic rats. Chin Med J (Engl) 2006;119:814-821

72. Shankland SJ, Ly H, Thai K, Scholey JW: Glomerular expression of tissue inhibitor of metalloproteinase (TIMP-1) in normal and diabetic rats. J Am Soc Nephrol 1996;7:97-104

73. Bhatt LK, Addepalli V: Attenuation of diabetic retinopathy by enhanced inhibition of MMP-2 and MMP-9 using aspirin and minocycline in streptozotocin-diabetic rats. Am J Transl Res 2010;2:181-189

74. Mohammad G, Kowluru RA: Matrix metalloproteinase-2 in the development of diabetic retinopathy and mitochondrial dysfunction. Lab Invest 2010;90:1365-1372

75. Mohammad G, Kowluru RA: Novel role of mitochondrial matrix metalloproteinase-2 in the development of diabetic retinopathy. Invest Ophthalmol Vis Sci 2011;52:3832-3841

76. Kowluru RA, Kanwar M: Oxidative stress and the development of diabetic retinopathy: contributory role of matrix metalloproteinase-2. Free Radic Biol Med 2009;46:1677-1685

77. Kowluru RA: Role of matrix metalloproteinase-9 in the development of diabetic retinopathy and its regulation by H-Ras. Invest Ophthalmol Vis Sci 2010;51:4320-4326

78. Suzuki D, Yagame M, Kim Y, Sakai H, Mauer M: Renal in situ hybridization studies of extracellular matrix related molecules in type 1 diabetes mellitus. Nephron 2002;92:564-572

79. Suzuki D, Miyazaki M, Jinde K, Koji T, Yagame M, Endoh M, Nomoto Y, Sakai H: In situ hybridization studies of matrix metalloproteinase-3, tissue inhibitor of metalloproteinase-1 and type IV collagen in diabetic nephropathy. Kidney Int 1997;52:111-119

80. Salzmann J, Limb GA, Khaw PT, Gregor ZJ, Webster L, Chignell AH, Charteris DG: Matrix metalloproteinases and their natural inhibitors in fibrovascular membranes of proliferative diabetic retinopathy. Br J Ophthalmol 2000;84:1091-1096

81. Thrailkill KM, Bunn RC, Moreau CS, Cockrell GE, Simpson PM, Coleman HN, Frindik JP, Kemp SF, Fowlkes JL: Matrix metalloproteinase-2 dysregulation in type 1 diabetes. Diabetes Care 2007;30:2321-2326

82. Gharagozlian S, Svennevig K, Bangstad HJ, Winberg JO, Kolset SO: Matrix metalloproteinases in subjects with type 1 diabetes. BMC Clin Pathol 2009;9:7

83. Toni M, Hermida J, Goni MJ, Fernandez P, Parks WC, Toledo E, Montes R, Diez N: Matrix metalloproteinase-10 plays an active role in microvascular complications in type 1 diabetic patients. Diabetologia 2013;56:2743-2752

84. Jacqueminet S, Ben Abdesselam O, Chapman MJ, Nicolay N, Foglietti MJ, Grimaldi A, Beaudeux JL: Elevated circulating levels of matrix metalloproteinase-9 in type 1 diabetic patients with and without retinopathy. Clin Chim Acta 2006;367:103-107

85. Nin JW, Jorsal A, Ferreira I, Schalkwijk CG, Prins MH, Parving HH, Tarnow L, Rossing P, Stehouwer CD: Higher plasma levels of advanced glycation end products are associated with incident cardiovascular disease and all-cause mortality in type 1 diabetes: a 12-year follow-up study. Diabetes Care 2011;34: 442-447 
86. Fukami K, Yamagishi S, Coughlan MT, Harcourt BE, Kantharidis P, Thallas-Bonke V, Okuda S, Cooper ME, Forbes JM: Ramipril inhibits AGE-RAGE-induced matrix metalloproteinase-2 activation in experimental diabetic nephropathy. Diabetol Metab Syndr 2014;6:86

87. Maillard: Action des acides aminés sur les sucres: formation des mélanoidines par voie méthodique. Compte-Rendu de l'Académie des Scienes 1912:66-68

88. Boel E, Selmer J, Flodgaard HJ, Jensen T: Diabetic late complications: will aldose reductase inhibitors or inhibitors of advanced glycosylation endproduct formation hold promise? J Diabetes Complications 1995;9:104-129

89. Baynes JW, Thorpe SR: Glycoxidation and lipoxidation in atherogenesis. Free Radic Biol Med 2000;28:1708-1716

90. Brownlee M: Biochemistry and molecular cell biology of diabetic complications. Nature 2001;414: 813-820

91. Monnier VM, Sell DR, Genuth S: Glycation products as markers and predictors of the progression of diabetic complications. Ann N Y Acad Sci 2005;1043:567-581

92. Miura J, Yamagishi S, Uchigata Y, Takeuchi M, Yamamoto H, Makita Z, Iwamoto Y: Serum levels of noncarboxymethyllysine advanced glycation endproducts are correlated to severity of microvascular complications in patients with Type 1 diabetes. J Diabetes Complications 2003;17:16-21

93. Nathan DM, Cleary PA, Backlund JY, Genuth SM, Lachin JM, Orchard TJ, Raskin P, Zinman B: Intensive diabetes treatment and cardiovascular disease in patients with type 1 diabetes. N Engl J Med 2005;353:2643-2653

94. Tonelli M, Wiebe N, Culleton B, House A, Rabbat C, Fok M, McAlister F, Garg AX: Chronic kidney disease and mortality risk: a systematic review. J Am Soc Nephrol 2006;17:2034-2047

95. van der Velde M, Matsushita K, Coresh J, Astor BC, Woodward M, Levey A, de Jong P, Gansevoort RT, Levey AS, de Jong PE, El-Nahas M, Eckardt KU, Kasiske BL, Ninomiya T, Chalmers J, Macmahon S, Tonelli M, Hemmelgarn B, Sacks F, Curhan G, Collins AJ, Li S, Chen SC, Hawaii Cohort KP, Lee BJ, Ishani A, Neaton J, Svendsen K, Mann JF, Yusuf S, Teo KK, Gao P, Nelson RG, Knowler WC, Bilo HJ, Joosten H, Kleefstra N, Groenier KH, Auguste P, Veldhuis K, Wang Y, Camarata L, Thomas B, Manley T: Lower estimated glomerular filtration rate and higher albuminuria are associated with all-cause and cardiovascular mortality. A collaborative meta-analysis of high-risk population cohorts. Kidney Int 2011;79:1341-1352

96. Libby P, Ridker PM, Hansson GK: Inflammation in atherosclerosis: from pathophysiology to practice. J Am Coll Cardiol 2009;54:2129-2138

97. Schram MT, Chaturvedi N, Schalkwijk CG, Fuller JH, Stehouwer CD: Markers of inflammation are crosssectionally associated with microvascular complications and cardiovascular disease in type 1 diabetes-the EURODIAB Prospective Complications Study. Diabetologia 2005;48:370-378

98. Targher G, Bertolini L, Zoppini G, Zenari L, Falezza G: Increased plasma markers of inflammation and endothelial dysfunction and their association with microvascular complications in Type 1 diabetic patients without clinically manifest macroangiopathy. Diabet Med 2005;22:999-1004

99. Devaraj S, Cheung AT, Jialal I, Griffen SC, Nguyen D, Glaser N, Aoki T: Evidence of increased inflammation and microcirculatory abnormalities in patients with type 1 diabetes and their role in microvascular complications. Diabetes 2007; 56:2790-2796

100. Astrup AS, Tarnow L, Pietraszek L, Schalkwijk CG, Stehouwer CD, Parving HH, Rossing P: Markers of endothelial dysfunction and inflammation in type 1 diabetic patients with or without diabetic nephropathy followed for 10 years: association with mortality and decline of glomerular filtration rate. Diabetes Care 2008;31:1170-1176

101. Chase AJ, Newby AC: Regulation of matrix metalloproteinase (matrixin) genes in blood vessels: a multistep recruitment model for pathological remodelling. J Vasc Res 2003;40:329-343

102. Newby AC: Dual role of matrix metalloproteinases (matrixins) in intimal thickening and atherosclerotic plaque rupture. Physiol Rev 2005;85:1-31

103. Stehouwer CD: Endothelial dysfunction in diabetic nephropathy: state of the art and potential significance for non-diabetic renal disease. Nephrol Dial Transplant 2004;19:778-781

104. Schalkwijk CG, Stehouwer CD: Vascular complications in diabetes mellitus: the role of endothelial dysfunction. Clin Sci (Lond) 2005;109:143-159 
105. Soedamah-Muthu SS, Chaturvedi N, Schalkwijk CG, Stehouwer CD, Ebeling P, Fuller JH: Soluble vascular cell adhesion molecule-1 and soluble E-selectin are associated with micro- and macrovascular complications in Type 1 diabetic patients. J Diabetes Complications 2006;20:188-195

106. Lopes-Virella MF, Carter RE, Gilbert GE, Klein RL, Jaffa M, Jenkins AJ, Lyons TJ, Garvey WT, Virella G: Risk factors related to inflammation and endothelial dysfunction in the DCCT/EDIC cohort and their relationship with nephropathy and macrovascular complications. Diabetes Care 2008;31:2006-2012

107. Giebel SJ, Menicucci G, McGuire PG, Das A: Matrix metalloproteinases in early diabetic retinopathy and their role in alteration of the blood-retinal barrier. Lab Invest 2005;85:597-607

108. Microvascular and acute complications in IDDM patients: the EURODIAB IDDM Complications Study. Diabetologia 1994;37:278-285

109. Chaturvedi N, Sjoelie AK, Porta M, Aldington SJ, Fuller JH, Songini M, Kohner EM: Markers of insulin resistance are strong risk factors for retinopathy incidence in type 1 diabetes. Diabetes Care 2001;24:284-289

110. Astrup AS, Tarnow L, Rossing P, Pietraszek L, Riis Hansen P, Parving HH: Improved prognosis in type 1 diabetic patients with nephropathy: a prospective follow-up study. Kidney Int 2005;68:1250-1257

111. Tarnow L, Cambien F, Rossing P, Nielsen FS, Hansen BV, Lecerf L, Poirier O, Danilov S, Parving HH: Lack of relationship between an insertion/deletion polymorphism in the angiotensin I-converting enzyme gene and diabetic nephropathy and proliferative retinopathy in IDDM patients. Diabetes 1995;44:489-494

112. Theilade S, Lajer M, Hansen TW, Joergensen C, Persson F, Andresdottir G, Reinhard H, Nielsen SE, Lacy P, Williams B, Rossing P: 24-hour central aortic systolic pressure and 24-hour central pulse pressure are related to diabetic complications in type 1 diabetes - a cross-sectional study. Cardiovasc Diabetol 2013;12:122

113. Theilade S, Lyngbaek S, Hansen TW, Eugen-Olsen J, Fenger M, Rossing P, Jeppesen JL: Soluble urokinase plasminogen activator receptor levels are elevated and associated with complications in patients with type 1 diabetes. J Intern Med 2014;

114. Porta M, Sjoelie AK, Chaturvedi N, Stevens L, Rottiers R, Veglio M, Fuller JH: Risk factors for progression to proliferative diabetic retinopathy in the EURODIAB Prospective Complications Study. Diabetologia 2001;44:2203-2209

115. Chaturvedi N, Schalkwijk CG, Abrahamian H, Fuller JH, Stehouwer CD: Circulating and urinary transforming growth factor beta1, Amadori albumin, and complications of type 1 diabetes: the EURODIAB prospective complications study. Diabetes Care 2002;25:2320-2327

116. Tarnow L, Cambien F, Rossing P, Nielsen FS, Hansen BV, Lecerf L, Poirier O, Danilov S, Boelskifte S, BorchJohnsen $\mathrm{K}$ : Insertion/deletion polymorphism in the angiotensin-I-converting enzyme gene is associated with coronary heart disease in IDDM patients with diabetic nephropathy. Diabetologia 1995;38:798-803 


\section{Chapter}

Serum levels of matrix metalloproteinase- $2,-3,-10$, and tissue inhibitor of metalloproteinase-1 are associated with vascular complications in patients with type 1 diabetes. The EURODIAB Prospective Complications Study C.D.A. Stehouwer; EURODIAB Prospective Complications Study Group Cardiovasc Diabetol. 2015;14:31 Erratum: Cardiovasc Diabetol. 2015;14:128 


\section{Abstract}

\section{Background}

Impaired regulation of extracellular matrix remodeling by matrix metalloproteinases (MMPs) and tissue inhibitor of metalloproteinase (TIMP) may contribute vascular complications in patients with type 1 diabetes. We investigated associations between serum MMP-1, -2, -3, -9, -10 and TIMP-1, and cardiovascular disease (CVD) or microvascular complications in type 1 diabetic patients. We also evaluated to which extent these associations could be explained by low-grade inflammation (LGI) or endothelial dysfunction (ED).

\section{Methods}

493 type 1 diabetes patients ( $39.5 \pm 9.9$ years old, $51 \%$ men) from the EURODIAB Prospective Complications Study were included. Linear regression analysis was applied to investigate differences in serum levels of MMP-1, -2, -3, -9, -10, and TIMP-1 between patients with and without CVD, albuminuria or retinopathy. All analyses were adjusted for age, sex, duration of diabetes, Hba1c and additionally for other cardiovascular risk factors including LGI and ED.

\section{Results}

Patients with CVD $(n=118)$ showed significantly higher levels of TIMP-1 $[\beta=0.32$ SD (95\% Cl: $0.12 ; 0.52)$ ], but not of MMPs, than patients without CVD ( $n=375)$. Higher serum levels of MMP-2, MMP-3, MMP-10 and TIMP-1 were associated with higher levels of albuminuria ( $p$-trends were $0.028,0.004,0.005$ and 0.001 , respectively). Severity of retinopathy was significantly associated with higher levels of MMP-2 ( -trend=0.017).

These associations remained significant after further adjustment for markers of LGI and ED.

\section{Conclusions}

These data support the hypothesis that impaired regulation of matrix remodeling by actions of MMP-2, -3 and -10 and TIMP-1 contributes to the pathogenesis of vascular complications in type 1 diabetes. 


\section{Background}

Type 1 diabetes is associated with an increased risk of macro- and microvascular disease. ${ }^{1,2}$ The exact mechanisms leading to vascular damage in type 1 diabetes have not been fully established, ${ }^{3}$ but impaired regulation of extracellular matrix (ECM) remodeling by matrix metalloproteinases (MMPs) may contribute to the development of vascular complications. ${ }^{4,5}$ Increased MMP activity has been associated with increased matrix turnover and potentially to pathological reorganization of the ECM in atherosclerosis, aneurysm formation, plaque disruption ${ }^{4}$ and, in diabetic nephropathy, to glomerular basement membrane thickening. ${ }^{5}$

MMPs are a group of zinc- and calcium-dependent endopeptidases, which degrade and rebuild proteins of the ECM, such as collagen, elastin, gelatin and casein. ${ }^{6}$ Thus far, more than 20 different MMPs have been identified, which, according to their various functions, can be divided into collagenases (e.g. MMP-1), gelatinases (MMP-2 and MMP-9), stromelysines (e.g. MMP-3 and MMP-10), matrilysines, and others. These enzymes can be excreted by various cells (e.g. fibroblasts, endothelial cells, monocytes and macrophages) or can be incorporated in the cellular membrane. Tissue inhibitors of metalloproteinases (TIMP-1-4) and $\alpha 2$-macroglobulin inhibit the action of MMPs. ${ }^{6}$

Plasma levels of MMPs have been observed to be higher in individuals with type 1 diabetes, compared to controls. ${ }^{7-9}$ The association between circulating MMPs and macrovascular disease in patients with type 1 diabetes has not been investigated, while studies on the associations between MMP levels and microvascular complications seem contradictory. For example, high levels of serum MMP-9 and a high MMP-9-to-TIMP ratio have been related to retinopathy, ${ }^{10}$ and high levels of plasma MMP-10 have been associated with nephropathy and proliferative retinopathy. ${ }^{8}$ In contrast, other studies did not show significant associations between plasma levels of MMP-2, -9 or TIMP-1 and microvascular complications. ${ }^{7,11}$ In fact, most studies were rather small,9-11 or limited to only one or two MMPs. ${ }^{7-11}$ In one large study of 269 type 1 diabetes patients and 269 non-diabetic controls, ${ }^{8}$ plasma MMP-10 was associated with nephropathy and proliferative retinopathy. However, plasma TIMP was not measured, whereas this may be of importance as MMP-10 activity is dependent on the amount of inhibitors. Nevertheless, in type 1 diabetes, hyperglycaemia, low-grade inflammation (LGI) and endothelial dysfunction (ED) have been found to be associated with higher plasma and tissue levels of MMPs and TIMP, ${ }^{5,9,12}$ and LGI and ED are consistently associated with macro- and microvascular complications. ${ }^{13-15}$

In view of these considerations, we hypothesized that high serum levels of MMPs and TIMP are related to macro- and microvascular complications in individuals with type 1 diabetes, possibly through associations with $L G I$ and ED. We investigated these hypotheses in a cross-sectional study of patients with type 1 diabetes, in whom we assessed macro- and microvascular disease and measured serum levels of MMP-1, -2, -3, $-9,-10$ and TIMP-1, as well as biomarkers of LGI and ED. 


\section{Methods}

\section{Study population}

In this analysis, data were used from the EURODIAB Prospective Complications Study, a European prospective cohort study that has been described in detail previously. ${ }^{16,17}$ In brief, baseline inclusion was performed between 1989 and 1991 in 3,250 patients with type 1 diabetes. Inclusion criteria were defined as a clinical diagnosis of type 1 diabetes before the age of 36 years, and requirement of continuous insulin therapy within the first year of diagnosis. Patients aged between 15 and 60 years were recruited from 31 centres in 16 European countries. Sample selection was stratified by sex, age group and duration of diabetes to ensure sufficient representation of all categories. These patients were invited for a follow-up visit 7-9 years after baseline enrolment. Of the 3,250 included patients, 1,880 (57.8\%) returned for re-examination. At follow-up, a cross-sectional nested case-control study was performed in a subset of patients $(n=543)$. Cases $(n=348)$ were those with one or more complications and controls $(n=195)$ were those with no evidence of complications. ${ }^{18}$ This type 1 diabetic patient control group was used to investigate differences in serum levels of MMPs and TIMP-1 compared with type 1 diabetic patients with vascular complications. For the current analyses, clinical data, serum samples for analysis of MMPs and TIMP-1 and information about potential confounders and mediators were available in 493 of these 543 patients. The study was approved by the local Ethics Committees and all patients gave informed consent.

\section{Main determinants}

Concentrations of MMPs and TIMP-1 were determined in serum samples that were stored at $-80^{\circ} \mathrm{C}$ after collection and in our laboratory until analyses and they were never thawed previously. Serum levels of MMP-1, MMP-2, MMP-3, MMP-9, MMP-10 and TIMP-1 were measured using a commercially available enzyme-linked immunosorbent assay (ELISA) kit [Human MMP 3-Plex Kit (for MMP-1, -3 and -9), Human MMP-2-Plex Kit (for MMP-2 and -10) and Human TIMP-1 Kit, MSD, Rockville, United States of America] according to the manufacturer's protocol. Serum samples of 10, 25 and 2.5 microliter were used for the MMP-3-plex kit, MMP-2-plex kit and TIMP-1 kit, respectively. The MMPs were detected in both a pro- and an active form. TIMP-1 was detected only in the active form. The intra- and inter-assay coefficients of variation were $7.0 \%$ and $8.0 \%$ for MMP-1, 4.5\% and 5.9\% for MMP-2, 8.4\% and 12.3\% for MMP-3, 5.3\% and $8.9 \%$ for MMP-9, 4.4\% and 9.7\% for MMP-10, and 4.3\% and 5.2\% for TIMP-1, respectively. 


\section{Main outcomes}

\section{Macrovascular disease}

Cardiovascular disease (CVD) was defined as a cardiovascular event in a patient's medical history, including myocardial infarction, angina, coronary artery bypass graft, stroke or ischaemic changes in a centrally Minnesota-coded ECG. ${ }^{19}$

\section{Microvascular disease}

Albumin excretion rates were measured from duplicate 24-hour urine collections. ${ }^{17}$ Micro- and macroalbuminuria were defined as an albumin excretion rate between 20 and $200 \mu \mathrm{g} / \mathrm{min}$, or above $200 \mu \mathrm{g} / \mathrm{min}$, respectively. We also estimated the glomerular filtration rate (eGFR) using the Chronic Kidney Disease Epidemiology Collaboration (CKD-EPI) equation. ${ }^{20}$

Retinopathy was assessed from retinal photographs according to the EURODIAB protocol; non-proliferative retinopathy was defined as the presence of one or more microaneurysms, hemorrhages, and/or hard exudates. Proliferative retinopathy was defined as presence of any new vessels, fibrous proliferations, pre-retinal haemorrhages, vitreous hemorrhages or photocoagulation scars. ${ }^{21}$

\section{Other variables}

Apart from demographic data, supplemental information about medication, smoking history, systolic blood pressure and duration of diabetes was collected. Body weight and height were measured in patients wearing indoor clothing without shoes. With these values body mass index (BMI) was calculated. Fasting blood samples were taken for measurements of lipid profile and glycemic control. Cholesterol and triglyceride levels were measured by enzymatic colorimetric tests, ${ }^{22} \mathrm{HDL}$ was measured directly. ${ }^{23}$ Friedewald's formula was used to calculate LDL levels. ${ }^{24}$ Glycated hemoglobin (HbA1c) was measured by a latex-enhanced turbidimetric immunoassay (Roche Products, Welwyn Garden City, UK). The reference range for this assay was 4.2-6.2\%.

\section{Markers of LGI}

Plasma levels of C-reactive protein (CRP) were measured with a highly sensitive ELISA, developed and validated in our own laboratory. ${ }^{18}$ Plasma levels of interleukin-6 (IL-6) and tumor necrosis factor- $\alpha$ (TNF- $\alpha$ ) were measured using commercially available ELISA kits (R\&D Systems, Oxon, U.K.). ${ }^{18}$ Intra- and inter-assay coefficients of variation were 3.9 and $8.7 \%$ for CRP, 4.5 and $9.0 \%$ for IL-6, and 7.3 and $8.5 \%$ for TNF- $\alpha$, respectively. 


\section{Markers of ED}

Plasma soluble E-selectin (sE-selectin) and soluble vascular cell adhesion molecule-1 (sVCAM-1) were measured by sandwich enzyme immunoassays (R\&D Systems, OXON, U.K.) in duplicate. Individual mean values of the duplicates were used in the ED scores. Intra- and inter-assay coefficients of variation were $2.1 \%$ and $3.1 \%$ for sE-selectin, and $4.0 \%$ and $9.1 \%$ for sVCAM-1, respectively.

\section{Statistical analyses}

All analyses were performed using the Statistical Package for Social Sciences (SPSS), version 20 (IBM Corporation, Armonk, NY, USA). Log transformation was performed for variables with a skewed distribution (triglycerides, serum creatinine, eGFR, MMP-1, MMP-2, MMP-3, MMP-9, MMP-10, CRP, IL-6 and TNF- $\alpha$ ). Student's t- or Chi-Square tests were performed for comparisons of characteristics between individuals with and without vascular complications, as appropriate.

Linear regression analyses were performed to examine the extent to which serum levels of MMP-1, MMP-2, MMP-3, MMP-9, MMP-10 and TIMP-1 differed between individuals with and without CVD or microvascular complications as well as to test for linear trends in serum levels of MMPs and TIMP-1 between both micro- and macroalbuminuria and between non-proliferative and proliferative retinopathy. Results of these analyses are presented as standardized regression coefficients to enable comparison of the magnitude of the associations. We have only investigated single MMPs and TIMP-1 in the analyses and have not adjusted for the presence of others, because each MMP has its own substrate specificity and actions.

Analyses were adjusted for age, sex, duration of diabetes and HbA1c (model 1). Further adjustments were made for LDL, HDL, systolic blood pressure, eGFR, antihypertensive medication, triglycerides, BMI and smoking, and for the presence of CVD, albuminuria and/or retinopathy, as appropriate (model 2). Markers of ED (model 3) and LGI (model 4) were subsequently added to this model to explore whether the association between serum levels of MMPs and TIMP-1 on the one hand and CVD and microvascular complications on the other (if any) were explained (i.e., potentially mediated) by LGI and/or ED. Markers of LGI were comprized into an overall LGI z-score by computing a z-score after averaging the z-scores of InCRP, InIL-6 and InTNF- $\alpha$. ED z-scores were expressed after averaging of the z-scores of SVCAM-1 and sE-selectin. A z-score represents the difference between the individual biomarker score and the mean value in the population, expressed in units of the standard deviation of the study population. This enables comparison of markers expressed in different units. The two scores represent a more robust value of the individual's levels of $L G I$ and $E D$, as they reduce the influence of biological variability expected when LGI and ED should be characterized by levels of each biomarker separately. ${ }^{25}$ 


\section{Results}

\section{Patients' characteristics}

Table 2.1 shows the characteristics of patients with or without vascular complications. Patients with vascular complications were characterized by higher age, BMI and systolic blood pressure. Levels of HbA1c, LDL, triglycerides, MMP-1, MMP-2, MMP-3, MMP-10, TIMP-1 and markers of LGI and ED were significantly higher in individuals with vascular complications compared to those without.

\section{Associations between MMPs, TIMP-1 and CVD}

Significantly higher serum levels of TIMP-1 $[\beta=0.27$ SD $(95 \% \mathrm{Cl}: 0.06 ; 0.48)]$ were observed in individuals with CVD $(n=118)$ as compared to those without, after adjustment for age, sex, duration of diabetes and HbA1c (Supplemental Table S2.1, model 1). The association became even stronger $[0.32(0.12 ; 0.52)]$ after further adjustment for other cardiovascular risk factors, albuminuria and retinopathy (Figure 2.1; Supplemental Table S2.1, model 2). In contrast, MMP-1, MMP-2, MMP-3, MMP-9 and MMP-10 did not differ between groups.

\section{CVD}

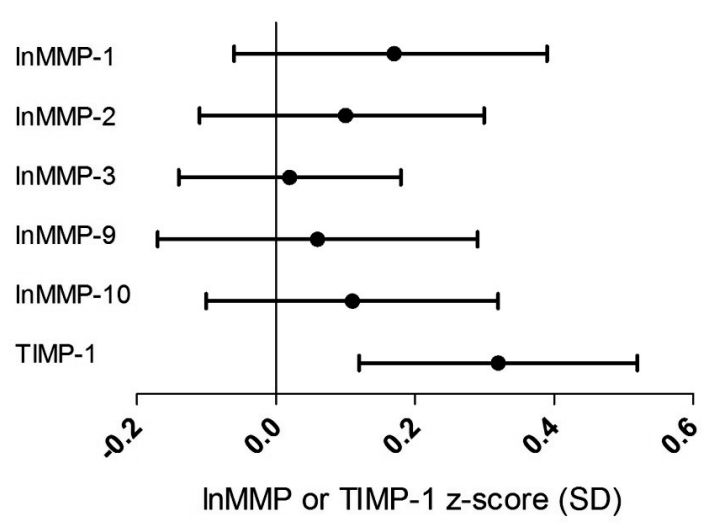

Figure 2.1 Associations between serum levels of MMPs, TIMP-1 and CVD

Point estimates and 95\% confidence intervals show the difference in serum levels of InMMP or TIMP-1 (in SD) in patients with vs. those without CVD resulting from a multivariable regression model including all cardiovascular risk factors, albuminuria and retinopathy (model 2) 
Table 2.1 Clinical characteristics of the study population

\begin{tabular}{|c|c|c|c|}
\hline & $\begin{array}{c}\text { Vascular } \\
\text { complications } \\
(n=306)\end{array}$ & $\begin{array}{c}\text { No vascular } \\
\text { complications } \\
(n=187)\end{array}$ & p-value \\
\hline Age (years) & $41.6(10.4)$ & $36.1(8.1)$ & $<0.001$ \\
\hline Sex (male/female, \%) & $54 / 46$ & $46 / 54$ & 0.096 \\
\hline $\operatorname{BMI}\left(\mathrm{kg} / \mathrm{m}^{2}\right)$ & $24.8(3.5)$ & $23.9(2.6)$ & $<0.001$ \\
\hline HbA1c (\%) & $9.0(1.6)$ & $7.7(1.3)$ & $<0.001$ \\
\hline $\mathrm{HbA} 1 \mathrm{c}(\mathrm{mmol} / \mathrm{mol})$ & $75(17.4)$ & $61(14.1)$ & $<0.001$ \\
\hline Duration of diabetes (years) & $25.2(8.9)$ & $15.5(7.0)$ & $<0.001$ \\
\hline LDL cholesterol (mmol/l) & $3.30(1.06)$ & $2.88(0.93)$ & $<0.001$ \\
\hline HDL cholesterol (mmol/l) & $1.59(0.42)$ & $1.68(0.45)$ & 0.032 \\
\hline Triglycerides (mmol/l) & $1.13[0.84-1.58]$ & 0.85 [0.67-1.09] & $<0.001$ \\
\hline Smoking (no/former/current) (\%) & $36 / 31 / 33$ & $46 / 26 / 28$ & 0.047 \\
\hline Systolic blood pressure (mmHg) & $127(21)$ & $115(13)$ & $<0.001$ \\
\hline Diastolic blood pressure (mmHg) & $75(12)$ & $73(10)$ & 0.085 \\
\hline Serum creatinine $(\mu \mathrm{mol} / \mathrm{l})$ & $76.0[68.0-90.0]$ & $71.0[64.0-79.0]$ & $<0.001$ \\
\hline eGFR $\left(\mathrm{ml} / \mathrm{min} / 1.73 \mathrm{~m}^{2}\right)$ & 95.7 [79.0-107.9] & $107.9[97.2-115.6]$ & $<0.001$ \\
\hline Cardiovascular disease (\%) & 38.6 & - & - \\
\hline Albuminuria (normo-/micro-/macro-)(\%) & $37.9 / 25.2 / 36.9$ & - & - \\
\hline Retinopathy (no/background/proliferative) (\%) & $11.4 / 40.9 / 47.7$ & - & - \\
\hline Antihypertensive medication (\%) & 45.4 & 5.9 & $<0.001$ \\
\hline ACE-inhibitor (\%) & 37.6 & 4.3 & $<0.001$ \\
\hline MMP-1 (ng/ml) & $12.8[7.0-19.9]$ & $10.8[5.9-16.5]$ & 0.006 \\
\hline MMP-2 (ng/ml) & 110 [100-120] & $103[96-110]$ & $<0.001$ \\
\hline MMP-3 (ng/ml) & $17.8[11.2-28.3]$ & $12.9[8.3-19.6]$ & $<0.001$ \\
\hline MMP-9 (ng/ml) & 122 [81-195] & $110[66-172]$ & 0.023 \\
\hline MMP-10 (pg/ml) & $1285[942-1931]$ & $1077[790-1635]$ & $<0.001$ \\
\hline TIMP-1 (ng/ml) & $313(100)$ & $256(74)$ & $<0.001$ \\
\hline C-reactive protein $(\mathrm{mg} / \mathrm{l})$ & $1.28[0.46-2.68]$ & $0.71[0.35-1.80]$ & $<0.001$ \\
\hline Interleukin-6 (pg/ml) & $2.12[1.35-3.86]$ & $1.57[1.06-2.50]$ & $<0.001$ \\
\hline Tumor necrosis factor- $\alpha$ (pg/ml) & $3.16[2.33-4.42]$ & $2.22[1.68-2.85]$ & $<0.001$ \\
\hline Soluble e-selectin (ng/ml) & $35.7(16.6)$ & $31.1(11.1)$ & $<0.001$ \\
\hline Soluble vascular cell adhesion molecule-1 (ng/ml) & $435(144)$ & $378(104)$ & $<0.001$ \\
\hline
\end{tabular}

Data are presented as means (standard deviation), median [inter-quartile range], or percentages, as appropriate. Vascular complication: presence of previous CVD, macroalbuminuria, or proliferative retinopathy or the combination of microalbuminuria and non-proliferative retinopathy; BMI, body mass index; HbA1C, glycated hemoglobin; LDL, low-density lipoprotein; HDL, high-density lipoprotein; eGFR, estimated glomerular filtration rate by CKD-EPI formula; MMP, matrix metalloproteinase; TIMP-1, tissue inhibitor of metalloproteinase-1

\section{Associations between MMPs, TIMP-1 and albuminuria}

Patients with microalbuminuria $(n=77)$ showed significantly higher serum levels of MMP-2 compared to patients with normal urinary albumin excretion after adjustment for cardiovascular risk factors and other vascular complications $[0.32(0.03 ; 0.61)]$ (Figure 2.2A; Supplemental Table S2.2, model 2). Macroalbuminuria ( $n=113)$, compared 
to normoalbuminuria, was associated with even higher levels of MMP-2 [0.38 (0.010; 0.66)] (Figure 2.2A; Supplemental Table S2.2). In this group, additionally, higher serum levels of MMP-3 [0.33 (0.11; 0.54)], MMP-10 [0.38 (0.08; 0.67)] and TIMP-1 [0.45 (0.18; 0.72)] were found. Significant associations with the degree of albuminuria (normo- vs. micro- vs. macroalbuminuria) were observed for higher serum levels of MMP-2 (p-trend $=0.028), \mathrm{MMP}-3$ (p-trend $=0.004), \mathrm{MMP}-10(\mathrm{p}$-trend $=0.005)$ and TIMP-1 $(\mathrm{p}$-trend $=$ 0.001) (Figure 2.2A).

\section{Associations between MMPs, TIMP-1 and retinopathy}

Proliferative retinopathy $(n=146)$ was associated with higher levels of MMP-2 [0.71 (0.45; 0.96)], MMP-3 [0.49 (0.28; 0.70)], MMP-10 [0.39 (0.11; 0.66)] and TIMP-1 [0.54 (0.28; 0.81)] after adjustment for age, sex, duration of diabetes and $\mathrm{HbA} 1 \mathrm{C}$ (Supplemental Table S2.3, model 1). However, after further adjustment for the other cardiovascular risk factors, CVD and albuminuria, only MMP-2 [0.36 (0.06; 0.66)] remained significantly higher in patients with proliferative retinopathy vs. those without retinopathy (Figure 2.2B; Supplemental Table S2.3, model 2). In addition, a significant trend across the severity of retinopathy was observed for increasing serum levels of MMP-2 (p-trend $=0.017$ ).

In contrast, non-proliferative retinopathy ( $\mathrm{n}=125)$ was not significantly associated with higher serum levels of MMPs or TIMP-1 (Figure 2.2B; Supplemental Table S2.3).
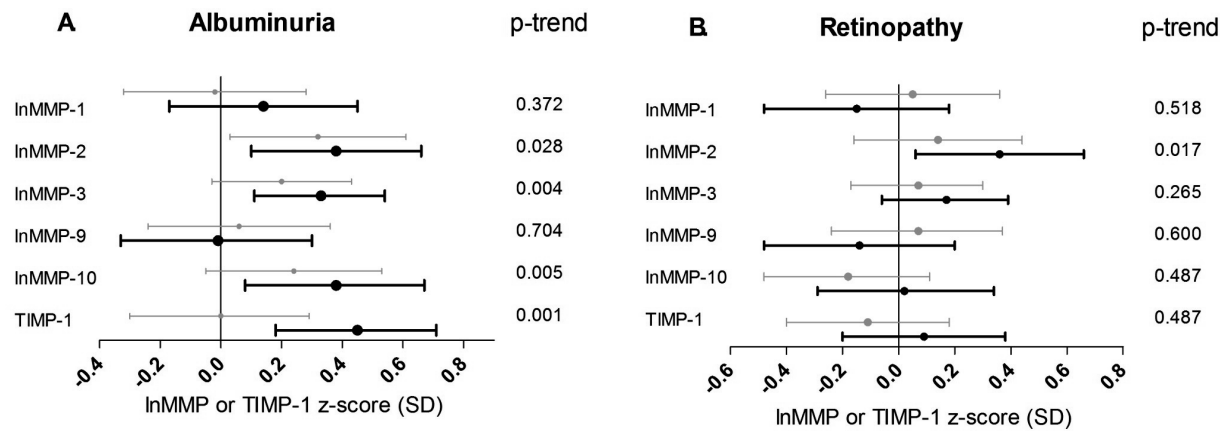

Figure 2.2 Associations between serum levels of MMPs, TIMP-1 and microvascular complications Point estimates and 95\% confidence intervals show the difference in serum levels of InMMP or TIMP-1 (in SD) in patients with vs. those without microvascular complications resulting from a multivariable regression model including all cardiovascular risk factors and the other vascular complications (model 2).

A, differences in micro- (grey bars) or macroalbuminuria (black bars) compared to normoalbuminuria; $\mathrm{B}$, differences in non-proliferative (grey bars) and proliferative retinopathy (black bars) compared to no retinopathy.

P-trend indicates the statistical significance of the associations between serum levels of MMPs or TIMP and the degrees of albuminuria (normo- vs. micro- vs. macroalbuminuria) or retinopathy (no vs. non-proliferative vs. proliferative retinopathy). 


\section{Associations of MMPs and TIMP-1 with LGI and ED}

Associations between MMPs and TIMP-1 and the individual markers of LGI and ED are shown in Supplemental Table S2.4. After adjustment for all cardiovascular risk factors (model 2), MMP-1, -3, -9, -10, and TIMP-1, but not MMP-2, were positively and significantly associated with the LGI score. In addition, MMP-2, MMP-10 and TIMP-1 were positively and significantly associated with the ED score (Table 2.2, model 2). However, significant associations between serum levels of MMPs and TIMP-1 on the one hand and CVD, albuminuria and retinopathy on the other hand were largely independent of LGI and ED, as these associations remained significant after adding the LGI and/or ED score to the model (Supplemental Table S2.1, S2.2 and S2.3; model 3, 4 and 5).

Table 2.2 Associations between InMMP-1, -2, -3, -9, -10 and TIMP-1 and the LGI and ED scores

\begin{tabular}{|c|c|c|c|c|c|c|c|}
\hline & \multirow[b]{2}{*}{ Model } & \multicolumn{3}{|c|}{ Inflammation (z-score) } & \multicolumn{3}{|c|}{ Endothelial dysfunction (z-score) } \\
\hline & & $\beta$ & $95 \% \mathrm{Cl}$ & $\mathrm{p}$-value & $\beta$ & $95 \% \mathrm{Cl}$ & p-value \\
\hline \multirow[t]{3}{*}{ MMP-1 } & 1 & 0.07 & $-0.02 ; 0.15$ & 0.112 & 0.00 & $-0.09 ; 0.09$ & 0.998 \\
\hline & 2 & 0.08 & $0.00 ; 0.16$ & 0.047 & 0.00 & $-0.09 ; 0.08$ & 0.987 \\
\hline & 3 & 0.08 & $-0.00 ; 0.15$ & 0.062 & -0.01 & $-0.09 ; 0.08$ & 0.834 \\
\hline \multirow[t]{3}{*}{ MMP-2 } & 1 & 0.10 & $0.01 ; 0.18$ & 0.032 & 0.19 & $0.10 ; 0.27$ & $<0.001$ \\
\hline & 2 & 0.03 & $-0.06 ; 0.12$ & 0.526 & 0.14 & $0.04 ; 0.23$ & 0.004 \\
\hline & 3 & 0.00 & $-0.08 ; 0.09$ & 0.950 & 0.13 & $0.04 ; 0.22$ & 0.006 \\
\hline \multirow[t]{3}{*}{ MMP-3 } & 1 & 0.26 & $0.16 ; 0.36$ & $<0.001$ & 0.08 & $-0.03 ; 0.19$ & 0.137 \\
\hline & 2 & 0.19 & $0.09 ; 0.30$ & 0.001 & -0.02 & $-0.14 ; 0.10$ & 0.715 \\
\hline & 3 & 0.17 & $0.06 ; 0.28$ & 0.003 & -0.04 & $-0.16 ; 0.08$ & 0.474 \\
\hline \multirow[t]{3}{*}{ MMP-9 } & 1 & 0.16 & $0.08 ; 0.25$ & $<0.001$ & -0.02 & $-0.11 ; 0.06$ & 0.592 \\
\hline & 2 & 0.17 & $0.09 ; 0.24$ & $<0.001$ & -0.02 & $-0.10 ; 0.07$ & 0.718 \\
\hline & 3 & 0.16 & $0.08 ; 0.24$ & $<0.001$ & -0.02 & $-0.10 ; 0.06$ & 0.577 \\
\hline \multirow[t]{3}{*}{ MMP-10 } & 1 & 0.24 & $0.16 ; 0.32$ & $<0.001$ & 0.13 & $0.04 ; 0.21$ & 0.003 \\
\hline & 2 & 0.22 & $0.14 ; 0.30$ & $<0.001$ & 0.11 & $0.02 ; 0.20$ & 0.019 \\
\hline & 3 & 0.21 & $0.13 ; 0.30$ & $<0.001$ & 0.10 & $0.01 ; 0.19$ & 0.029 \\
\hline \multirow[t]{3}{*}{ TIMP-1 } & 1 & 0.34 & $0.27 ; 0.43$ & $<0.001$ & 0.25 & $0.17 ; 0.33$ & $<0.001$ \\
\hline & 2 & 0.29 & $0.21 ; 0.38$ & $<0.001$ & 0.21 & $0.12 ; 0.30$ & $<0.001$ \\
\hline & 3 & 0.28 & $0.20 ; 0.37$ & $<0.001$ & 0.20 & $0.11 ; 0.29$ & $<0.001$ \\
\hline
\end{tabular}

$\beta$, standardized regression coefficient: indicates increase in the low-grade inflammation and endothelial dysfunction score (in SDs) per 1 SD increase in InMMP-1, -2, -3, -9, -10 or TIMP-1. Cl: confidence interval. MMP: matrix metalloproteinase.

Model 1: adjusted for age, sex, duration of diabetes and $\mathrm{HbA1C}$

Model 2: model 1 + BMI, triglycerides, LDL, HDL, systolic blood pressure, eGFR, smoking and antihypertensive medication

Model 3: model $2+C V D$, albuminuria and retinopathy

\section{Additional analyses}

Adding use of ACE inhibitors specifically versus antihypertensive medication in general as covariable to our models, did not materially influence the results. Similarly, adjustment 
for urinary albumin excretion rate instead of albuminuria status and waist-hip-ratio instead of BMI did not change our results (data not shown). We have not included adjustment for TIMP-1 in model 2 in the main analyses between serum levels of MMPs and vascular complications, because TIMP-1 levels are influenced by all MMPs and not just by one MMP. Therefore, we have also not analyzed associations between MMP/TIMP-1 ratios and vascular complications. However, additional adjustment in model 2 for TIMP-1 levels did also not materially influence our results.

In the relationship between MMPs, TIMP and vascular complications, eGFR (a marker of renal dysfunction) can be considered as a potential confounder or mediator. If eGFR should be a mediator rather than a confounder, models including eGFR may be overadjusted. We therefore reanalyzed the data without adjustment for eGFR; results were generally similar to models with such adjustment (data not shown).

\section{Discussion}

This is the first study that comprehensively assessed associations of MMPs and TIMP-1 with long-term complications in type 1 diabetes. In a group of 493 patients with and without diabetes complications, we found that high serum levels of TIMP-1 were associated with CVD. In addition, MMP-2, -3 and -10, and TIMP-1 levels were associated with macroalbuminuria, and MMP-2 was associated with proliferative retinopathy. All these associations remained significant after adjustment for cardiovascular risk factors, prevalence of other vascular complications and markers of LGI and ED.

\section{MMPs, TIMP-1 and CVD}

Prevalent CVD was associated with higher serum levels of TIMP-1, but not of MMP-1, -2, $-3,-9$ and -10. In accordance, significantly higher levels of plasma TIMP-1 have been previously observed in two studies in type 2 diabetic patients with a history of coronary artery disease. ${ }^{26,27}$ In addition, in a large prospective cohort study of individuals with and without (presumably type 2) diabetes, increased plasma TIMP-1 levels, measured six months after myocardial infarction, was significantly associated with left ventricular remodeling and adverse outcomes. ${ }^{28}$ In the current study on individuals with type 1 diabetes, measurements of serum TIMP-1 were, by its cross-sectional design, performed 1 month to 10 years after the cardiovascular event. Although we cannot prove a relationship between elevated serum levels of TIMP-1 and new cardiovascular events, TIMP-1 may be a marker for abnormal regulation of matrix remodeling after a cardiovascular event. This is supported by studies in animal models showing elevation of plasma TIMP-1 in type 1 diabetic minipigs with left ventricular hypertrophy, increased cardiac fibrosis and cardiac dysfunction. ${ }^{29}$ 


\section{MMPs, TIMP-1 and albuminuria}

Serum levels of MMP-2 were significantly higher in both micro- and macroalbuminuric patients compared to those with normoalbuminuria. In contrast, a previous study in type 1 diabetic patients did not show increased levels of MMP-2 in microalbuminuric vs. normoalbuminuric individuals. ${ }^{7}$ This discrepancy may be explained by the very small sample size $(n=12)$ of that study. ${ }^{7}$

Thus far, no studies have been reported regarding circulating MMP-3 and diabetic nephropathy in humans or animals. Our study is the first study showing that higher serum levels of MMP-3 are associated with increasing levels of albuminuria. In accordance with our study, in type 2 diabetic patients, high interstitial MMP-3 mRNA expression was associated with higher degrees of interstitial injury. ${ }^{30}$ The increased serum MMP-3 could therefore potentially originate from the renal interstitium. This finding may be biologically explained by the fact that type IV collagen, one of the major substrates of proteolysis by MMP-3, is an important component of the basal lamina of the glomerular basement membrane, Bowman's capsule, and of the basal membranes of renal tubules and vessels. ${ }^{31}$ MMP-3-dependent type IV collagen proteolysis therefore constitutes a potential explanation for the current observation of a strong association between MMP-3 and albuminuria.

In addition, higher serum MMP-10 levels were significantly associated with macroalbuminuria in our study. This result is in full agreement with a recent study ${ }^{8}$ in patients with type 1 diabetes, in which a higher plasma level of MMP-10 was associated with diabetic nephropathy independently of classical risk factors. In addition, MMP-10 knockout (streptozotocin (STZ)-induced) diabetic mice showed lower renal mesangial cell expansion and lower renal macrophage influx compared to wildtype diabetic mice, ${ }^{8}$ suggesting that MMP-10 may be involved in the onset of diabetic nephropathy.

Besides MMP-2, -3, and -10, TIMP-1 was also associated with macroalbuminuria. This association was also observed in one-sided nephrectomized type 1 diabetic rats compared to non-diabetic controls. ${ }^{32}$ These results reflect a possible role for TIMP-1 in increased renal fibrosis in early diabetic nephropathy.

\section{MMPs, TIMP-1 and retinopathy}

Serum MMP-2 levels showed a significant positive correlation with proliferative retinopathy. Salzmann et al. ${ }^{33}$ showed an overall increase of different MMPs (-2, -3 and -9) and TIMP-1 in specimens of retinal tissue of patients with proliferative diabetic retinopathy compared to retinal tissue of patients without diabetes. Also, increased retinal levels of MMP-2 were found in rats and mice with STZ-induced diabetes compared to non-diabetic controls. ${ }^{34}$ Our current clinical results, supported by the relatively scarce literature, indicate that MMP-2 may play a role the pathogenesis of proliferative diabetic retinopathy. In addition, the study by Toni et al. ${ }^{8}$ has shown a significant association between plasma MMP-10 and proliferative retinopathy, which is 
in accordance with our current findings in the minimally adjusted model (including age, sex, duration of diabetes and $\mathrm{HbA1c}$ ). However, this was no longer significant after additional adjustment for cardiovascular risk factors and the presence of CVD and albuminuria. Since Toni et al. did not adjust for the presence of other vascular complications, this could explain the observed difference between their and our study. We did not find a significant association of serum MMPs or TIMP-1 levels with nonproliferative retinopathy. This is in contrast with the results of the study of Jacqueminet et al., ${ }^{10}$ in which increased serum MMP-9 levels were shown in patients with type 1 diabetes with minimal to moderate retinopathy $(n=14)$ compared to diabetic patients without retinopathy and non-diabetic controls. In addition, potential confounders may not have been fully addressed in this earlier study, but were shown to be of importance in the current study. In fact, also the current study showed a significant association between serum MMP-9 levels and the presence of non-proliferative retinopathy in the crude model [standardized $\beta=0.22(0.00 ; 0.44)$ ], but this was attenuated after adjustment for confounding.

\section{MMPs, TIMP-1, LGI and ED}

In our study, all MMPs, except MMP-2, were significantly correlated with markers of low-grade inflammation (Table 2.2, model 2). Indeed, it is well known that MMP-2 has a more potent anti-inflammatory effect compared to other MMPs, which may be attributable to the fact that monocyte chemo-attractant protein-3 (MCP-3) is an important substrate of MMP-2. The product of MCP-3 after cleavage by MMP-2 antagonizes the effect of MCP-3 on the chemokine receptors-1, -2 and -3 and may thereby attenuate the inflammatory response. ${ }^{35}$ Although MMP-1 and MMP-3 are known to cleave MCP-1, -2 and -4, the antagonistic effects of the cleaved products on inflammation are minimal, because of lower affinity for the chemokine receptors. ${ }^{36}$ MMPs can be activated and up-regulated by mediators of inflammation, but also have an intrinsic effect on the inflammatory response. ${ }^{37}$ Although the exact role of MMPs in the inflammatory cascade remains incompletely understood, our results suggest that associations of MMPs and TIMP-1 with complications of type 1 diabetes are unlikely to be explained by LGI.

MMP-2, MMP-10 and TIMP-1 were associated with markers of ED. These findings are in line with literature data demonstrating that MMP-2 is able to degrade occludin, a tight junction protein, in the blood retinal membrane leading to increased vascular permeability, $^{12}$ and that MMP-10 can induce endothelial proteolysis in response to CRP. $^{38}$ In addition, plasma TIMP-1 levels are associated with endothelial cell migration. ${ }^{39}$ Thus, associations between MMP and TIMP-1 levels and ED are biologically plausible; nevertheless, our results suggest that associations of MMPs and TIMP-1 with complications of type 1 diabetes are unlikely to be explained by ED. 


\section{Limitations}

There are several limitations to our study. First, the cross-sectional design only allows speculations on causality. We thus cannot exclude that increased levels of serum MMPs and TIMP-1 may be a result instead of a cause of vascular complications. Second, circulating levels of biomarkers may reflect specific organ injury, but can also originate from other tissues. We adjusted for other known vascular complications, but cannot rule out that other causative factors may contribute and the observed associations could therefore be underestimated. In addition, MMP-1, -3, -10 and TIMP-1 have a smaller molecular weight than albumin ${ }^{40,41}$ and may be filtered in the glomerulus, especially in albuminuric patients, which may also have underestimated the associations between these biomarkers and vascular complications. Third, serum samples can be obtained easily and we thus used serum levels to investigate the involvement of MMPs and TIMP-1 in the pathogenesis of vascular complications in type 1 diabetes. However, we do not know whether serum markers truly reflect the local pathological situation at the tissue level. Finally, our results suggest that associations of MMPs and TIMP-1 with complications of type 1 diabetes are unlikely to be explained by LGI or ED, but we cannot exclude roles of LGI or ED not reflected by the biomarkers we used.

\section{Conclusion}

In this study with type 1 diabetic patients, serum MMP-2, MMP-3, MMP-10 and TIMP-1 levels are associated with macro- and microvascular complications and these associations were largely independent of LGI and ED. Further (prospective) studies are warranted to elucidate the involvement of MMPs and TIMPs in the development of diabetic macro- and microvascular complications. 


\section{References}

1. Laing SP, Swerdlow AJ, Slater SD, Burden AC, Morris A, Waugh NR, Gatling W, Bingley PJ, Patterson CC: Mortality from heart disease in a cohort of 23,000 patients with insulin-treated diabetes. Diabetologia 2003;46:760-765

2. Soedamah-Muthu SS, Fuller JH, Mulnier HE, Raleigh VS, Lawrenson RA, Colhoun HM: High risk of cardiovascular disease in patients with type 1 diabetes in the U.K.: a cohort study using the general practice research database. Diabetes Care 2006;29:798-804

3. Snell-Bergeon JK, Nadeau K: Cardiovascular disease risk in young people with type 1 diabetes. J Cardiovasc Transl Res 2012;5:446-462

4. Galis ZS, Khatri JJ: Matrix metalloproteinases in vascular remodeling and atherogenesis: the good, the bad, and the ugly. Circ Res 2002;90:251-262

5. Kadoglou NP, Daskalopoulou SS, Perrea D, Liapis CD: Matrix metalloproteinases and diabetic vascular complications. Angiology 2005;56:173-189

6. Visse R, Nagase $\mathrm{H}$ : Matrix metalloproteinases and tissue inhibitors of metalloproteinases: structure, function, and biochemistry. Circ Res 2003;92:827-839

7. Thrailkill KM, Bunn RC, Moreau CS, Cockrell GE, Simpson PM, Coleman HN, Frindik JP, Kemp SF, Fowlkes JL: Matrix metalloproteinase-2 dysregulation in type 1 diabetes. Diabetes Care 2007;30:2321-2326

8. Toni M, Hermida J, Goni MJ, Fernandez P, Parks WC, Toledo E, Montes R, Diez N: Matrix metalloproteinase-10 plays an active role in microvascular complications in type 1 diabetic patients. Diabetologia 2013;56:2743-2752

9. Gharagozlian S, Svennevig K, Bangstad HJ, Winberg JO, Kolset SO: Matrix metalloproteinases in subjects with type 1 diabetes. BMC Clin Pathol 2009;9:7

10. Jacqueminet S, Ben Abdesselam O, Chapman MJ, Nicolay N, Foglietti MJ, Grimaldi A, Beaudeux JL: Elevated circulating levels of matrix metalloproteinase-9 in type 1 diabetic patients with and without retinopathy. Clin Chim Acta 2006;367:103-107

11. Thrailkill KM, Moreau CS, Cockrell GE, Jo CH, Bunn RC, Morales-Pozzo AE, Lumpkin CK, Fowlkes JL: Disease and gender-specific dysregulation of NGAL and MMP-9 in type 1 diabetes mellitus. Endocrine 2010;37:336-343

12. Giebel SJ, Menicucci G, McGuire PG, Das A: Matrix metalloproteinases in early diabetic retinopathy and their role in alteration of the blood-retinal barrier. Lab Invest 2005;85:597-607

13. Soedamah-Muthu SS, Chaturvedi N, Schalkwijk CG, Stehouwer CD, Ebeling P, Fuller JH: Soluble vascular cell adhesion molecule-1 and soluble E-selectin are associated with micro- and macrovascular complications in Type 1 diabetic patients. J Diabetes Complications 2006;20:188-195

14. Costacou T, Lopes-Virella MF, Zgibor JC, Virella G, Otvos J, Walsh M, Orchard TJ: Markers of endothelial dysfunction in the prediction of coronary artery disease in type 1 diabetes. The Pittsburgh Epidemiology of Diabetes Complications Study. J Diabetes Complications 2005;19:183-193

15. Devaraj S, Cheung AT, Jialal I, Griffen SC, Nguyen D, Glaser N, Aoki T: Evidence of increased inflammation and microcirculatory abnormalities in patients with type 1 diabetes and their role in microvascular complications. Diabetes 2007;56:2790-2796

16. Chaturvedi N, Sjoelie AK, Porta M, Aldington SJ, Fuller JH, Songini M, Kohner EM: Markers of insulin resistance are strong risk factors for retinopathy incidence in type 1 diabetes. Diabetes Care 2001;24:284-289

17. Chaturvedi N, Bandinelli S, Mangili R, Penno G, Rottiers RE, Fuller JH: Microalbuminuria in type 1 diabetes: rates, risk factors and glycemic threshold. Kidney Int 2001;60:219-227

18. Schram MT, Chaturvedi N, Schalkwijk C, Giorgino F, Ebeling P, Fuller JH, Stehouwer CD: Vascular risk factors and markers of endothelial function as determinants of inflammatory markers in type 1 diabetes: the EURODIAB Prospective Complications Study. Diabetes Care 2003;26:2165-2173

19. Koivisto VA, Stevens LK, Mattock M, Ebeling P, Muggeo M, Stephenson J, Idzior-Walus B: Cardiovascular disease and its risk factors in IDDM in Europe. EURODIAB IDDM Complications Study Group. Diabetes Care 1996;19:689-697 
20. Levey AS, Stevens LA, Schmid CH, Zhang YL, Castro AF, 3rd, Feldman HI, Kusek JW, Eggers P, Van Lente F, Greene T, Coresh J: A new equation to estimate glomerular filtration rate. Ann Intern Med 2009;150:604-612

21. Aldington SJ, Kohner EM, Meuer S, Klein R, Sjolie AK: Methodology for retinal photography and assessment of diabetic retinopathy: the EURODIAB IDDM complications study. Diabetologia 1995;38:437-444

22. Fossati $\mathrm{P}$, Prencipe $\mathrm{L}$ : Serum triglycerides determined colorimetrically with an enzyme that produces hydrogen peroxide. Clin Chem 1982;28:2077-2080

23. Sugiuchi H, Uji Y, Okabe H, Irie T, Uekama K, Kayahara N, Miyauchi K: Direct measurement of highdensity lipoprotein cholesterol in serum with polyethylene glycol-modified enzymes and sulfated alphacyclodextrin. Clin Chem 1995;41:717-723

24. Friedewald WT, Levy RI, Fredrickson DS: Estimation of the concentration of low-density lipoprotein cholesterol in plasma, without use of the preparative ultracentrifuge. Clin Chem 1972;18:499-502

25. Nin JW, Ferreira I, Schalkwijk CG, Prins MH, Chaturvedi N, Fuller JH, Stehouwer CD: Levels of soluble receptor for AGE are cross-sectionally associated with cardiovascular disease in type 1 diabetes, and this association is partially mediated by endothelial and renal dysfunction and by low-grade inflammation: the EURODIAB Prospective Complications Study. Diabetologia 2009;52:705-714

26. Opstad TB, Pettersen AA, Weiss TW, Akra S, Ovstebo R, Arnesen H, Seljeflot I: Genetic variation, geneexpression and circulating levels of matrix metalloproteinase- 9 in patients with stable coronary artery disease. Clin Chim Acta 2012;413:113-120

27. Derosa G, Cicero AF, Scalise F, Avanzini MA, Tinelli C, Peros E, Fogari E, D'Angelo A: Metalloproteinases in diabetics and nondiabetics during acute coronary syndromes and after 3 months. Endothelium 2007; 14:175-183

28. Kelly D, Khan SQ, Thompson M, Cockerill G, Ng LL, Samani N, Squire IB: Plasma tissue inhibitor of metalloproteinase-1 and matrix metalloproteinase-9: novel indicators of left ventricular remodelling and prognosis after acute myocardial infarction. Eur Heart J 2008;29:2116-2124

29. Lu L, Zhang Q, Pu LJ, Peng WH, Yan XX, Wang L, Chen QJ, Zhu ZB, Michel JB, Shen WF: Dysregulation of matrix metalloproteinases and their tissue inhibitors is related to abnormality of left ventricular geometry and function in streptozotocin-induced diabetic minipigs. Int J Exp Pathol 2008;89:125-137

30. Suzuki D, Miyazaki M, Jinde K, Koji T, Yagame M, Endoh M, Nomoto Y, Sakai H: In situ hybridization studies of matrix metalloproteinase-3, tissue inhibitor of metalloproteinase-1 and type IV collagen in diabetic nephropathy. Kidney Int 1997;52:111-119

31. Hosoyamada Y, Sakai T: Structural arrangement of collagen fibrils in the periarterial connective tissue of the kidney: their functional relevance as a structural stabilizer against arterial pressure. Anat Sci Int 2012;87:80-87

32. Alter ML, Kretschmer A, Von Websky K, Tsuprykov O, Reichetzeder C, Simon A, Stasch JP, Hocher B: Early urinary and plasma biomarkers for experimental diabetic nephropathy. Clin Lab 2012;58:659-671

33. Salzmann J, Limb GA, Khaw PT, Gregor ZJ, Webster L, Chignell AH, Charteris DG: Matrix metalloproteinases and their natural inhibitors in fibrovascular membranes of proliferative diabetic retinopathy. Br J Ophthalmol 2000;84:1091-1096

34. Mohammad G, Kowluru RA: Matrix metalloproteinase-2 in the development of diabetic retinopathy and mitochondrial dysfunction. Lab Invest 2010;90:1365-1372

35. McQuibban GA, Gong JH, Tam EM, McCulloch CA, Clark-Lewis I, Overall CM: Inflammation dampened by gelatinase A cleavage of monocyte chemoattractant protein-3. Science 2000;289:1202-1206

36. McQuibban GA, Gong JH, Wong JP, Wallace JL, Clark-Lewis I, Overall CM: Matrix metalloproteinase processing of monocyte chemoattractant proteins generates CC chemokine receptor antagonists with anti-inflammatory properties in vivo. Blood 2002;100:1160-1167

37. Chase AJ, Newby AC: Regulation of matrix metalloproteinase (matrixin) genes in blood vessels: a multistep recruitment model for pathological remodelling. J Vasc Res 2003;40:329-343

38. Montero I, Orbe J, Varo N, Beloqui O, Monreal JI, Rodriguez JA, Diez J, Libby P, Paramo JA: C-reactive protein induces matrix metalloproteinase-1 and -10 in human endothelial cells: implications for clinical and subclinical atherosclerosis. J Am Coll Cardiol 2006;47:1369-1378

39. Akahane T, Akahane M, Shah A, Connor CM, Thorgeirsson UP: TIMP-1 inhibits microvascular endothelial cell migration by MMP-dependent and MMP-independent mechanisms. Exp Cell Res 2004;301:158-167 
40. Raffetto JD, Khalil RA: Matrix metalloproteinases and their inhibitors in vascular remodeling and vascular disease. Biochem Pharmacol 2008;75:346-359

41. Brew K, Nagase H: The tissue inhibitors of metalloproteinases (TIMPs): an ancient family with structural and functional diversity. Biochim Biophys Acta 2010;1803:55-71 


\section{Supplemental data}

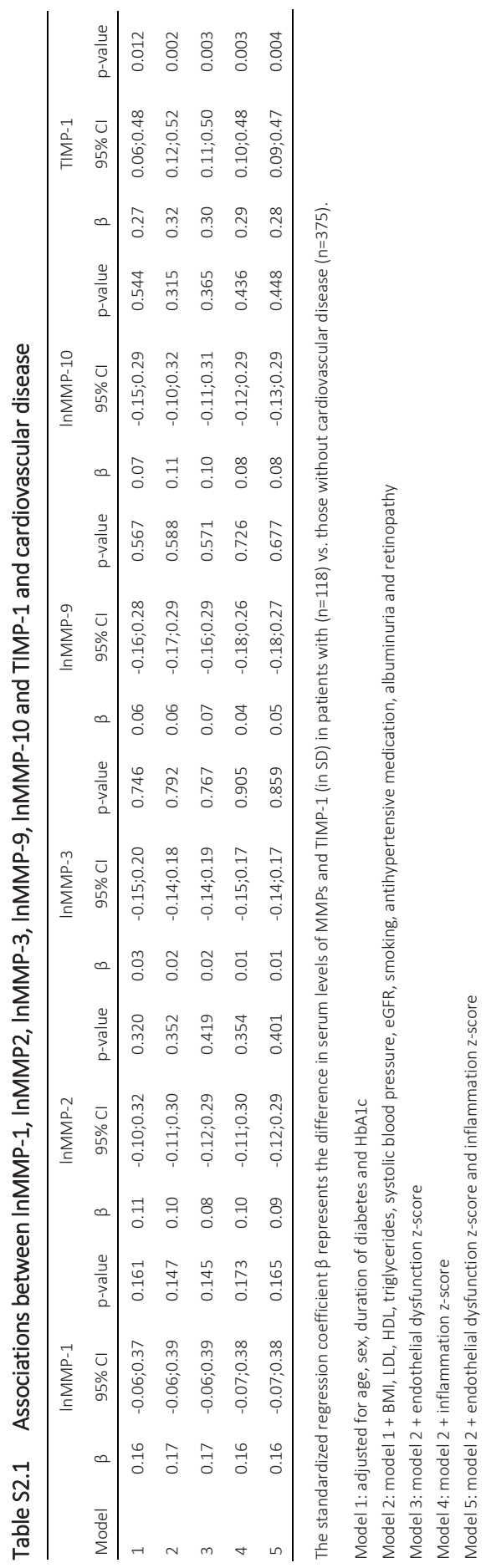




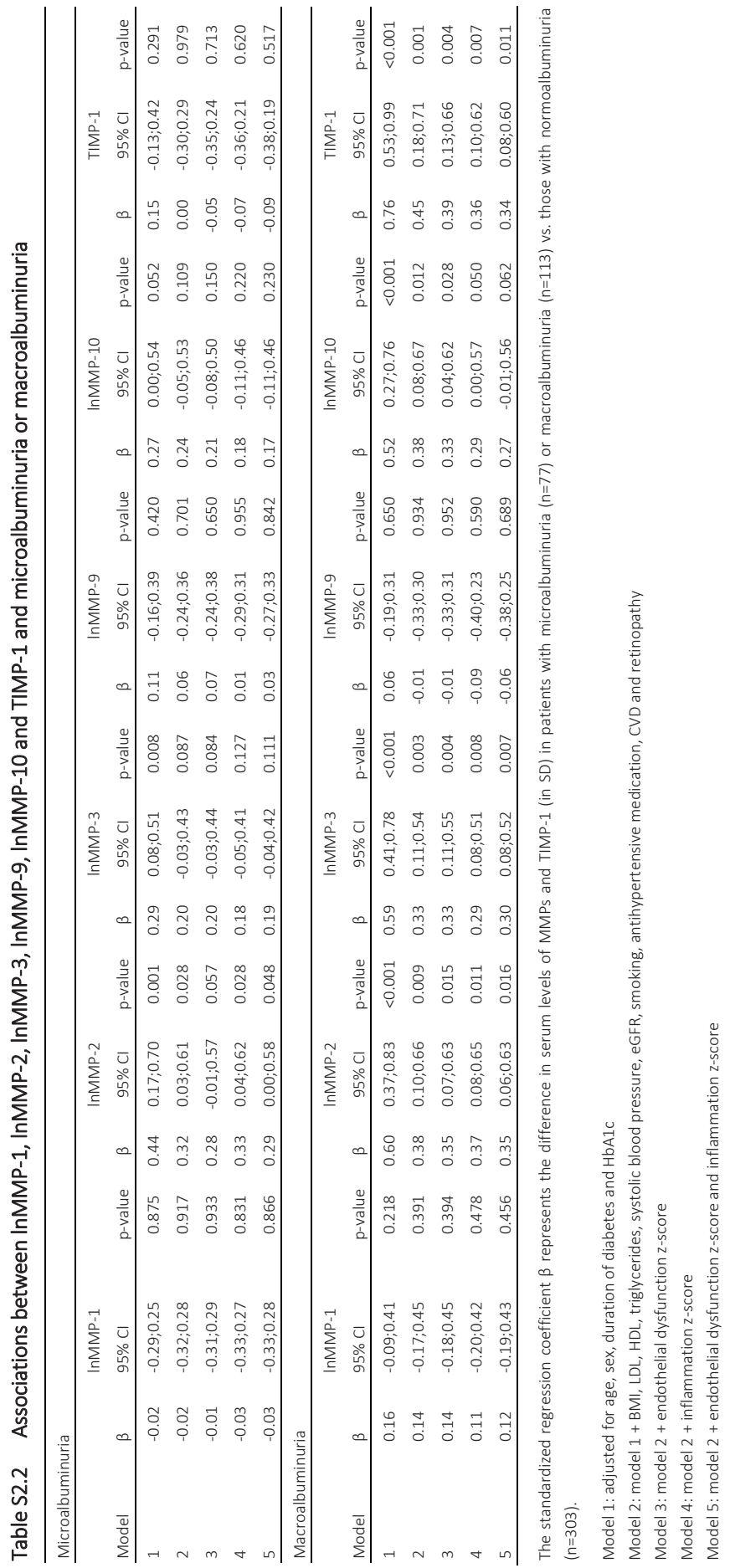




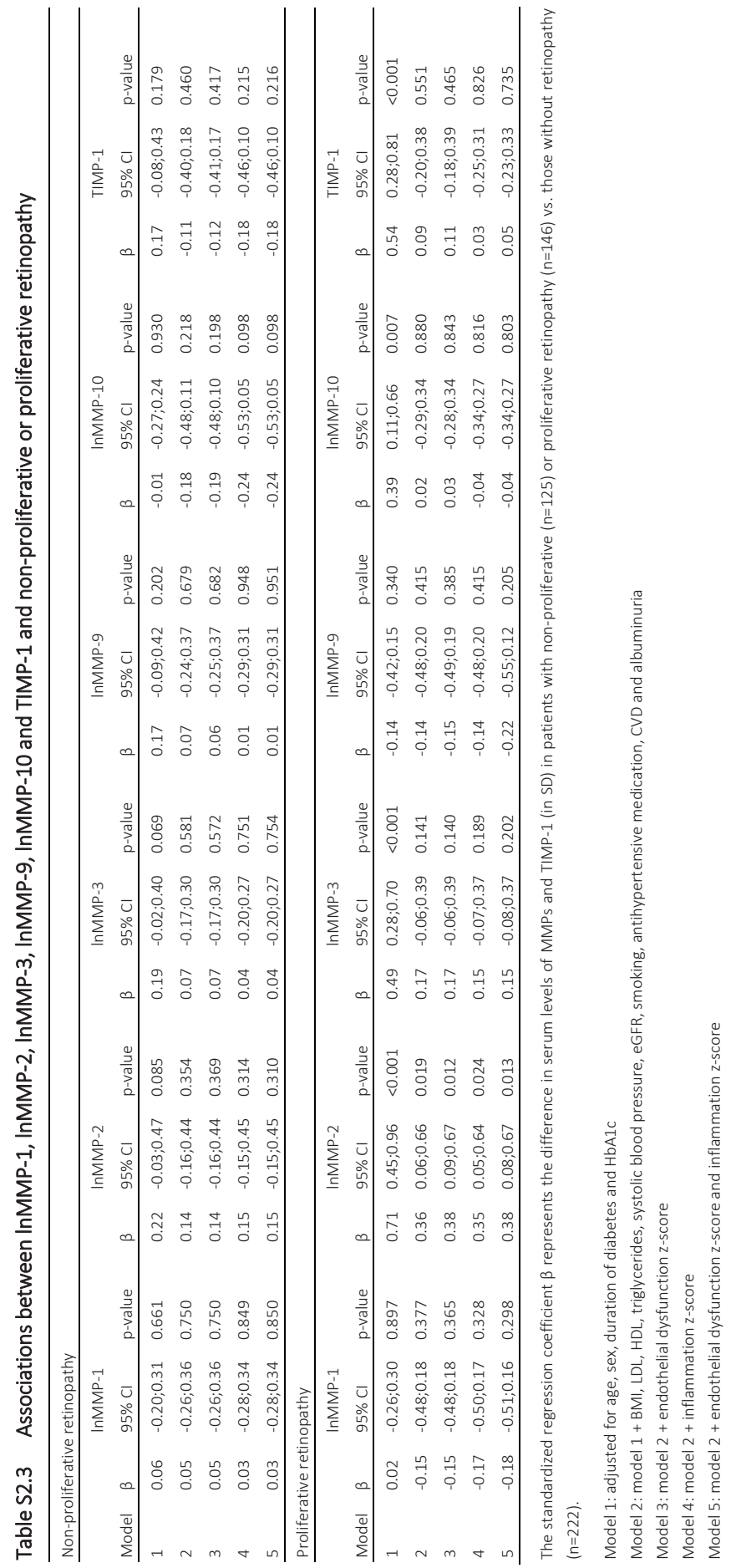




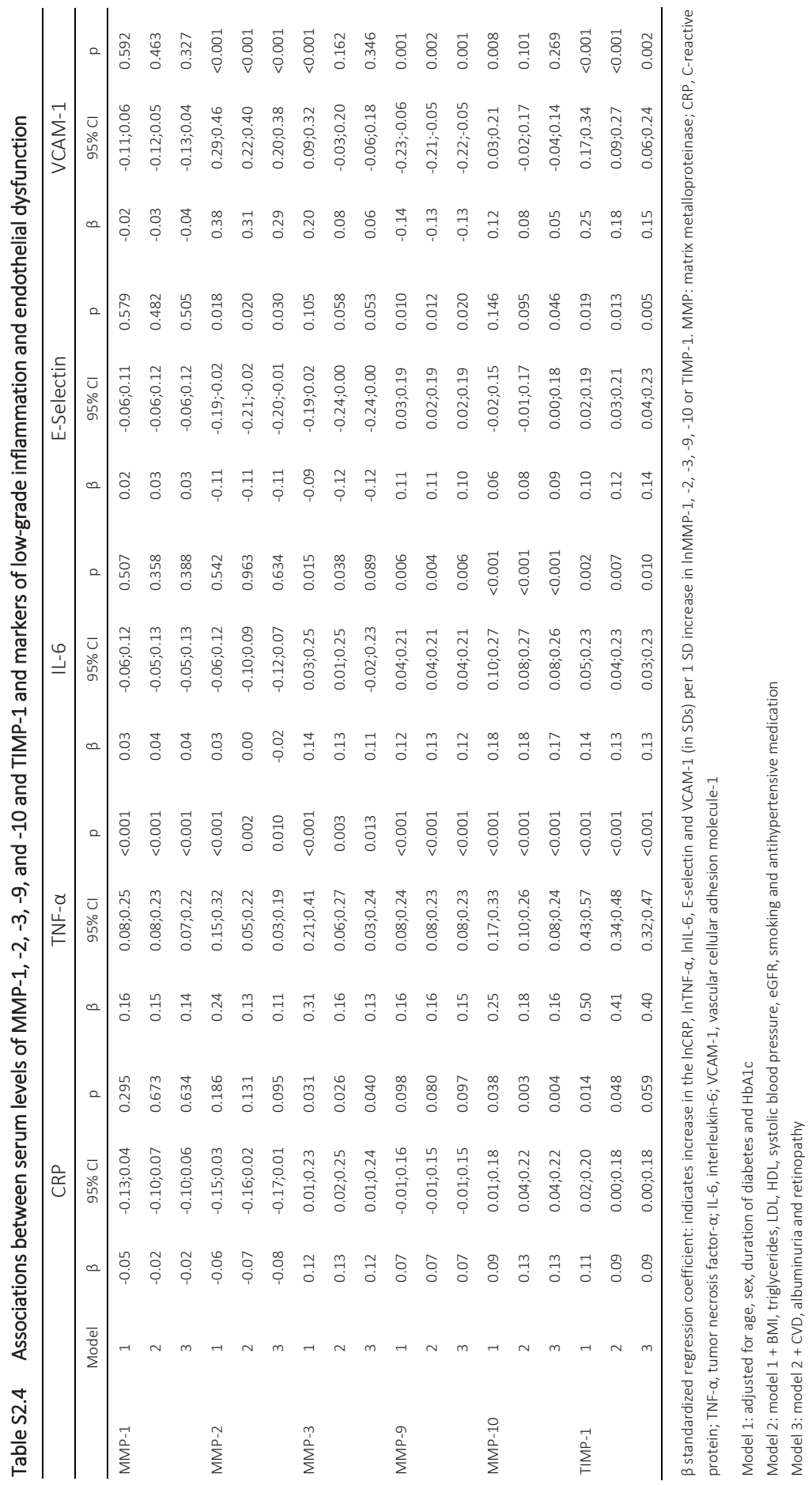





\section{Chapter}

Plasma matrix metalloproteinases are associated with incident cardiovascular disease and all-cause mortality in patients with type 1 diabetes: a 12-yr follow-up study 


\section{Abstract}

\section{Background}

Altered regulation of extracellular matrix remodeling by matrix metalloproteinases (MMPs) and tissue inhibitor of metalloproteinase (TIMP) may contribute to vascular complications in type 1 diabetes. We investigated associations between plasma MMP-1, $-2,-3,-9,-10$ and TIMP-1, and cardiovascular events and all-cause mortality in type 1 diabetic patients.

\section{Methods}

We prospectively followed 337 type 1 diabetic patients (mean age 41.4 years (9.6), 39\% female), 170 with and 167 without diabetic nephropathy, with median follow-up of 12.3 years. Survival analyses were applied to investigate differences in plasma MMP-1, $-2,-3,-9,-10$, and TIMP-1-levels in patients with and without a cardiovascular event and in those who died vs survivors. All analyses were adjusted for age, sex, duration of diabetes, $\mathrm{HbA1C}$, nephropathy and for other conventional cardiovascular risk factors.

\section{Results}

After adjustment for potential confounders, higher MMP-2 plasma levels were significantly associated with higher incidence of cardiovascular events [HR $1.49(95 \% \mathrm{Cl}$ 1.11;1.99)], and higher plasma levels of MMP-1 [1.38 (1.07;1.78)], MMP-2 [1.60 $(1.19 ; 2.15)]$ and MMP-3 $[1.39(1.05 ; 1.85)]$ were associated with all-cause mortality. All associations were independent of low-grade inflammation and endothelial dysfunction as estimated by plasma markers. Associations between MMP-2 and cardiovascular events and between MMP-3 and mortality were attenuated after further adjustment for eGFR and changes in eGFR.

\section{Conclusions}

Higher levels of MMP-2 are associated with CVD and higher MMP-1, -2 and -3 with allcause mortality. In addition, associations between MMP-2 and CVD, and MMP-3 and mortality were attenuated after adjustment for eGFR while both MMPs were associated with eGFR decline, indicating a possible mediating role of eGFR. 


\section{Background}

The pathophysiological mechanisms leading to cardiovascular disease (CVD) in type 1 diabetes ${ }^{1}$ have only been partly elucidated. Recent data suggest that altered regulation of extracellular matrix (ECM) remodeling by matrix metalloproteinases (MMPs) could play a role. ${ }^{2}$ MMPs are proteases, which degrade ECM components in normal and pathological conditions. Increased plasma MMP-2, for example, has been associated with macrovascular disease by increasing vascular remodeling as well as thrombus formation. ${ }^{3,4}$ MMP production may be enhanced by hyperglycemia, pro-inflammatory mediators, reactive oxygen species and aldosterone; ${ }^{3}$ MMP activity is dependent on the number of MMPs and their inhibitors, such as tissue inhibitor of metalloproteinase (TIMP) and a2-macroglobulin. ${ }^{5}$ In turn, increased activity of MMP-2 and MMP-9 is associated with low-grade inflammation (LGI) ${ }^{6}$ and endothelial dysfunction (ED) ${ }^{7}$, and increased MMP-9 levels have been associated with renal dysfunction. ${ }^{8}$ Therefore, LGI, ED and renal dysfunction constitute potential pathophysiological mechanisms through which MMPs could lead to CVD.

Interestingly, higher plasma levels of MMP-2 and -10 have been observed in patients with type 1 diabetes compared to non-diabetic controls., ${ }^{9,10}$ In cross-sectional studies, individuals with type 1 diabetes and retinopathy or nephropathy showed significantly higher circulating levels of MMP-9 and -10 compared to individuals without these complications. ${ }^{10,11}$ These data support the concept that MMPs may play an important role in the development of CVD. In addition, several prospective studies in (mainly) nondiabetic individuals have shown independent associations between plasma MMP- $2^{12}$ MMP-9 ${ }^{13}$ and TIMP-1 $1^{14}$ and CVD, as well as associations between MMP-1, ${ }^{15}$ MMP- $2^{12,16,17}$ and $-3^{18,19}$ and all-cause mortality. However, to our knowledge, no prospective studies have been reported on the associations between plasma MMPs or TIMP levels and CVD or all-cause mortality in type 1 diabetes.

In view of these considerations, we investigated associations, in type 1 diabetes, between plasma MMP-1, -2, -3, -9 and -10, and TIMP-1 and incident non-fatal and fatal cardiovascular events, as well as all-cause mortality, in a prospective study with more than 12 years of follow-up. In addition, we investigated the extent to which LGI, ED and renal dysfunction contributed to these associations.

\section{Methods}

\section{Study population and design}

In 1993, 199 type 1 diabetic patients with diabetic nephropathy and older than 18 years were enrolled in a prospective observational study at the outpatient clinic at Steno Diabetes Center. Diabetic nephropathy was defined as persistent macroalbuminuria 
(urinary albumin excretion (UAE) $>300 \mathrm{mg} / 24 \mathrm{~h}$ ) in at least two out of three previous consecutive 24-h urine collections, presence of retinopathy, and absence of other kidney or urinary tract disease. In addition, 192 normoalbuminuric diabetic patients (UAE $<30 \mathrm{mg} / 24 \mathrm{~h}$ ), matched for age, sex, and duration of diabetes, were enrolled for comparison. ${ }^{20}$

Patients with prior CVD ( $n=24)$, who were lost to follow-up ( $n=17)$, with end-stage renal disease at baseline $(n=7)$ and those with missing data on MMPs/TIMP-1 $(n=6)$ were excluded from the present analysis resulting in a total study population of 337 individuals. Patient selection and inclusion criteria have been described in detail elsewhere. ${ }^{21}$ The study was approved by the local ethics committee, in accordance with the Helsinki Declaration, and all patients gave their written informed consent.

\section{Baseline investigations}

Commercially available ELISA kits were used to measure levels of MMP-1, MMP-2, MMP-3, MMP-9, MMP-10 and TIMP-1 in EDTA plasma samples [Human MMP 3-Plex Kit (for MMP-1, -3 and -9), Human MMP-2-Plex Kit (for MMP-2 and -10) and Human TIMP-1 Kit, MSD, Rockville, United States of America], according to the manufacturer's protocol. The MMPs were detected in both pro- and active form. TIMP-1 was detected in the active form. The intra- and inter-assay coefficients of variation were $6.6 \%$ and $9.7 \%$ for MMP-1, 3.7\% and 7.1\% for MMP-2, 7.2\% and 14.1\% for MMP-3, 4.6\% and $13.7 \%$ for MMP-9, 2.9\% and 7.6\% for MMP-10, and 5.1\% and 7.4\% for TIMP-1, respectively.

\section{Other baseline measurements}

Measurements of other biomarkers and risk factors have been described in detail elsewhere. ${ }^{20}$ All clinical investigations were performed in the morning after an overnight fast. In $88 \%$ of the normoalbuminuric and $24 \%$ of macroalbuminuric patients antihypertensive medication was never prescribed. Patients using antihypertensive medication were asked to stop their antihypertensive and diuretic treatment eight days before the examination, but $15 \%$ of the 337 patients ( $27 \%$ in the nephropathy group) considered in our analyses did not comply with this recommendation.

Arterial blood pressure was measured twice, with an appropriate cuff size, following at least a 10-minute rest in supine position. Mean arterial pressure (MAP) was calculated as [systolic blood pressure $+(2 *$ diastolic blood pressure $)] / 3$. Body mass index (BMI) was defined as weight $(\mathrm{kg})$ divided by height $(\mathrm{m})$ squared. An ELISA was used to measure UAE from 24-h urine collections. Serum creatinine was determined by a kinetic Jaffé method and we estimated the glomerular filtration rate (eGFR) using the Chronic Kidney Disease Epidemiology Collaboration (CKD-EPI) equation, ${ }^{22}$ after creatinine was IDMS standardized. ${ }^{23}$ Patients were divided into three groups according to their smoking status: never, former or current smokers. 
An in-house ELISA was used to determine high-sensitivity C-reactive protein (CRP) ${ }^{24}$ and commercially available ELISA kits were used to measure plasma levels of secreted phospholipase A2 (sPLA2), interleukin-6 (IL-6), soluble vascular cell adhesion molecule-1 (sVCAM-1) and soluble intercellular adhesion molecule-1 (sICAM-1).

\section{Follow-up and study end-points}

All patients were followed up to the last visit at Steno Diabetes Center, until September $1^{\text {st }} 2006$, death $(n=83$ ) or emigration $(n=3)$. All patients were traced through the national register during autumn 2006. When a patient died before 1 September 2006, the date of death was recorded and the primary cause of death was obtained from the death certificate, which was reviewed by two independent observers. Additionally, information from necropsy reports was included, when available. All deaths were classified as cardiovascular unless an unequivocal non-cardiovascular cause was established. At the end of follow up, non-fatal events were retrieved from patients' records at Steno Diabetes Center or other hospital records. The primary endpoint of this study was fatal or non-fatal CVD (i.e. myocardial infarction, percutaneous coronary intervention, coronary bypass grafting, amputation due to ischemia, vascular surgery for peripheral atherosclerotic disease and stroke). Ischemic heart disease was examined through a WHO questionnaire combined with an ECG. ${ }^{20}$ In several patients, who were alive at follow-up and had more than one cardiovascular event during follow-up, the first event was used in the analyses. All-cause mortality was the secondary end-point.

\section{Statistical analyses}

All analyses were performed with SPSS version 20 for Windows (SPSS, Chicago, IL, USA). Triglycerides, UAE, CRP, IL-6, SPLA2, MMP-1, MMP-2, MMP-3, MMP-9, MMP-10 and TIMP-1 showed a skewed distribution and were loge transformed prior to further analyses. Student's t or Chi-square tests were performed for comparisons of baseline characteristics between groups, when appropriate. Cox proportional hazards regression models were applied to investigate associations between InMMP-1, InMMP-2, InMMP-3, InMMP-9, InMMP-10 and InTIMP-1 and study endpoints with adjustment for sex, age, duration of diabetes, $\mathrm{HbA} 1 \mathrm{c}$ and nephropathy-no nephropathy status (model 1). Further adjustments were performed for BMI, MAP, total cholesterol, smoking status, antihypertensive treatment and whether or not patients withheld their medication prior to baseline examinations (model 2). We used z-scores (in SD) of InMMPs and InTIMP-1 to enable direct comparisons between the strengths of the associations (if any) between the various markers and outcome measures.

An LGI score was calculated as the z-score of the average of the z-scores of InIL-6, InCRP, sICAM-1, and InsPLA2. ${ }^{2,25-27}$ Similarly, an ED score was calculated as the z-score of the average of the $z$-scores of sICAM- 1 and SVCAM-1. 2,26,27 We examined the cross-sectional associations between InMMP-1, InMMP-2, InMMP-3, InMMP-9, InMMP-10 and 
InTIMP-1, and renal function (i.e. eGFR and In-UAE), the LGI score and the ED score using linear regression analyses. Further analyses with adjustment of the associations between MMPs and TIMP-1 and study endpoints for eGFR, and In-UAE (model 3), LGI (model 4) and ED (model 5) were performed to evaluate the extent to which these pathophysiological processes could explain the associations between MMPs/TIMP-1 and study outcomes. Additionally, in case renal function does explain part of the association between circulating MMPs and TIMP-1 and outcome measures, the possible additional influence of decrease in eGFR over time will also be investigated. Finally, we investigated whether or not any such associations differed between patients with and without nephropathy or between men and women by adding interaction terms between nephropathy or sex and MMPs or TIMP-1 to the models. Whenever a p-value $<0.1$ was observed, results were presented for both groups separately.

\section{Results}

Supplemental Figure S3.1 depicts the flowchart concerning patient selection. During a median follow-up of 12.3 years [interquartile range: 7.7-12.5], 86 patients had a nonfatal $(n=54)$ or fatal $(n=49)$ cardiovascular event. Eighty-three patients died from various causes: cardiovascular $(n=49)$, diabetes $(n=4)$, infection $(n=13)$, cancer $(n=6)$, suicide $(n=4)$ and other $(n=7)$. Patients with a cardiovascular event or who died during follow-up were significantly older, had a longer duration of diabetes, higher levels of HbA1c and a higher prevalence of other cardiovascular risk factors (Table 3.1). Additionally, MMP-1, MMP-2, MMP-3, MMP-9, MMP-10 and TIMP-1 were higher in this group compared to patients without cardiovascular events or surviving the period of follow-up. Supplemental Table S3.1 shows the baseline characteristics according to nephropathyno nephropathy status. Supplemental Table S3.2 shows the correlations between five MMPs and TIMP-1.

\section{Associations of MMPs and TIMP-1 with incident CVD}

Supplemental Figure S3.2 shows the cumulative hazard plots of cardiovascular events according to tertiles of InMMPs and InTIMP-1. After adjustment for age, sex, duration of diabetes, HbA1C and nephropathy, higher levels of MMPs 1, 2, 3 and 9, and TIMP-1 were significantly associated with incidence of cardiovascular events, with a hazard ratio (HR) of 1.51 (95\%Cl 1.20;1.91) per 1 SD higher InMMP-1; 1.65 (1.28;2.13) for InMMP-2; 1.47 $(1.13 ; 1.92)$ for InMMP-3; $1.44(1.17 ; 1.78)$ for InMMP-9; and $1.87(1.40 ; 2.49)$ for InTIMP-1, respectively (Table 3.2 , model 1 ). 


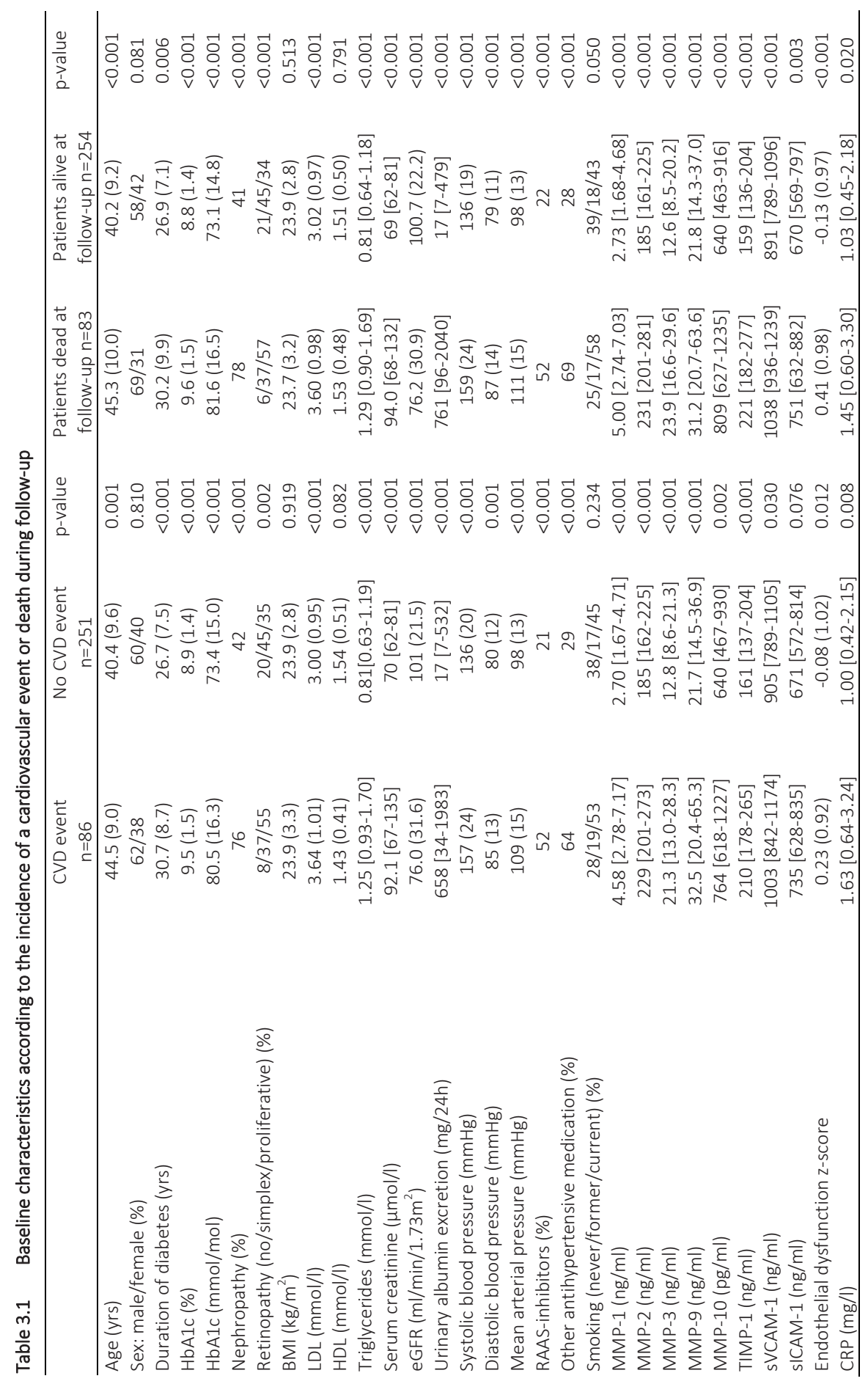



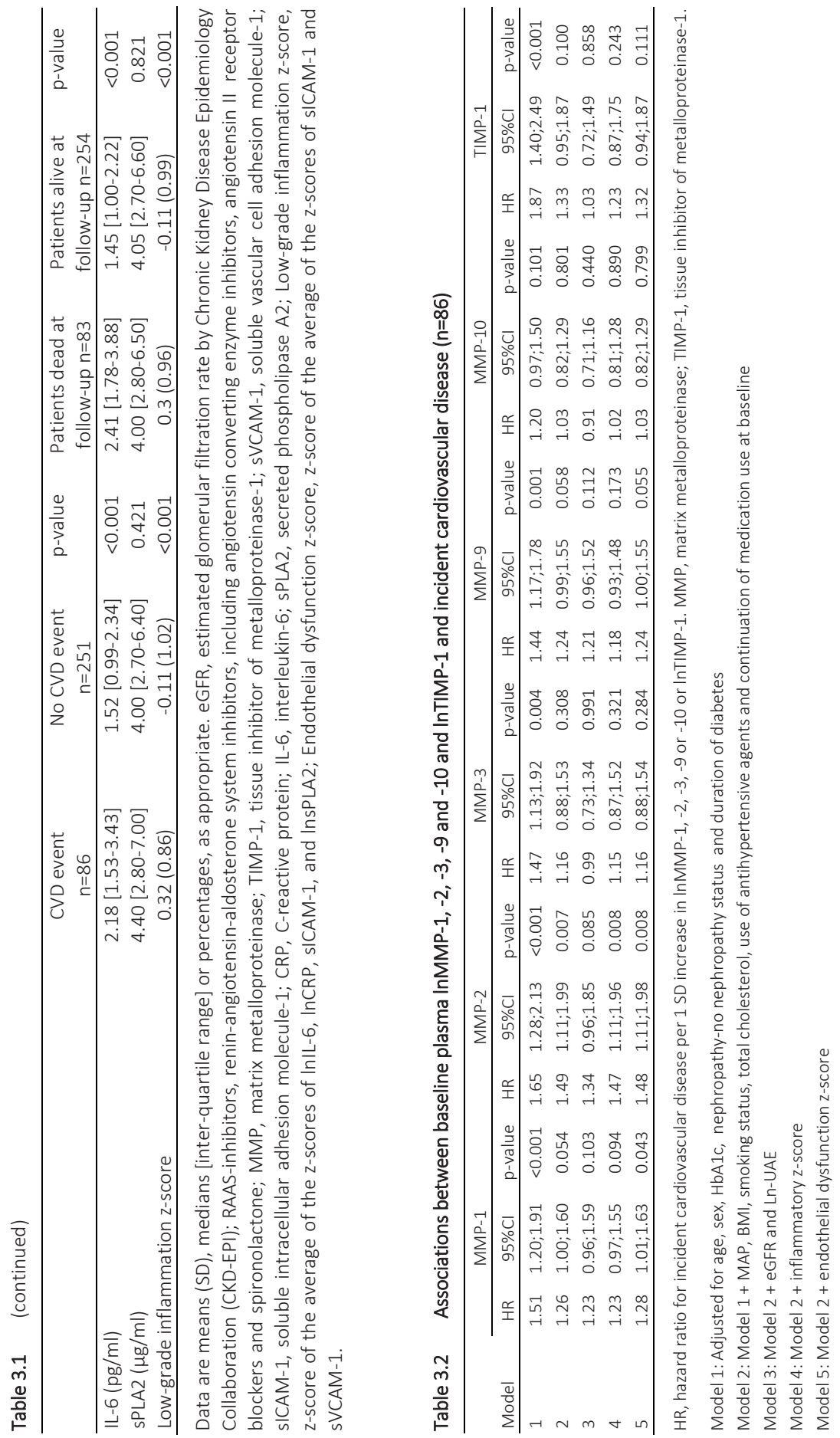
LnMMP-10 was not significantly associated with incident CVD. After further adjustment for other cardiovascular risk factors, antihypertensive treatment and continuation of antihypertensive treatment at baseline, higher InMMP-2 [1.49 (1.11;1.99)] remained significantly associated with the incidence of cardiovascular events (Table 3.2, model 2). This HR remained significant after further adjustment for markers of LGI and ED (Table 3.2, model 4 and 5). However, further adjustment for eGFR and InUAE (Table 3.2, model $3)$ decreased the HR from $1.49(1.11 ; 1.99)$ to $1.34(0.96 ; 1.85)$. This decline was explained mainly by the adjustment for eGFR, which decreased the HR to $1.36(0.98 ; 1.88)$, whereas adjustment for InUAE decreased the HR to $1.46(1.08 ; 1.98)$.

\section{Associations of MMPs and TIMP-1 with all-cause mortality}

Supplemental Figure S3.3 shows the cumulative hazard plots of all-cause mortality according to tertiles of InMMPs and InTIMP-1. After adjustment for age, sex, duration of diabetes, HbA1c and nephropathy, higher levels of MMPs 1, 2, 3, 9 and 10, and TIMP-1 were significantly associated with all-cause mortality, with HRs of $1.62(1.28 ; 2.06)$ per 1 SD higher InMMP-1; 1.93 (1.48;2.51) for InMMP-2; 1.82 (1.38;2.41) for InMMP-3; 1.39 $(1.14 ; 1.69)$ for InMMP-9; $1.33(1.06 ; 1.67)$ for InMMP-10; and $2.10(1.55 ; 2.85)$ for InTIMP-1, respectively (Table 3.3, model 1). After adjustment for other cardiovascular risk factors, antihypertensive treatment and continuation of antihypertensive treatment at baseline, higher InMMP-1 [1.38 (1.07;1.78)], InMMP-2 [1.60 (1.19;2.15)] and InMMP-3 [1.39 (1.05;1.85)] remained significantly associated with all-cause mortality (Table 3.3, model 2). These associations were independent of eGFR, UAE, LGI and ED (Table 3.3, models 3-5), except for the association between InMMP-3 and all-cause mortality, where the HR decreased from $1.39(1.05 ; 1.85)$ to $1.32(0.95 ; 1.83)$ after adjustment for eGFR and InUAE (Table 3.3, model 3). This decrease was explained mainly by the adjustment for eGFR, which decreased the HR to $1.32(0.96 ; 1.84)$, whereas adjustment for InUAE decreased the HR to $1.36(1.02 ; 1.82)$. The associations between MMP-1, MMP-2 and MMP-3 and all-cause mortality did not materially change after mutual adjustments for each of the other MMPs, except for the association between MMP-3 and all-cause mortality, which decreased after adjustment for MMP-1 [to 1.29 (0.96; 1.73)] and MMP-2 [to $1.20(0.88 ; 1.62)]$.

\section{Associations of MMPs and TIMP-1 with eGFR, InUAE, LGI and ED}

Cross-sectionally and after full adjustment for cardiovascular risk factors, InMMP-1, -2, 3, -10 and InTIMP-1 were significantly and inversely associated with eGFR at baseline (Table 3.4, model 2). LnMMP-2, -3, -10 and InTIMP-1 were significantly and positively associated with InUAE (Table 3.4, model 2). LGI was significantly associated with InMMP1, -9 and InTIMP-1, and ED was significantly associated with InMMP-1 and InTIMP-1 (Table 3.4, model 2). 


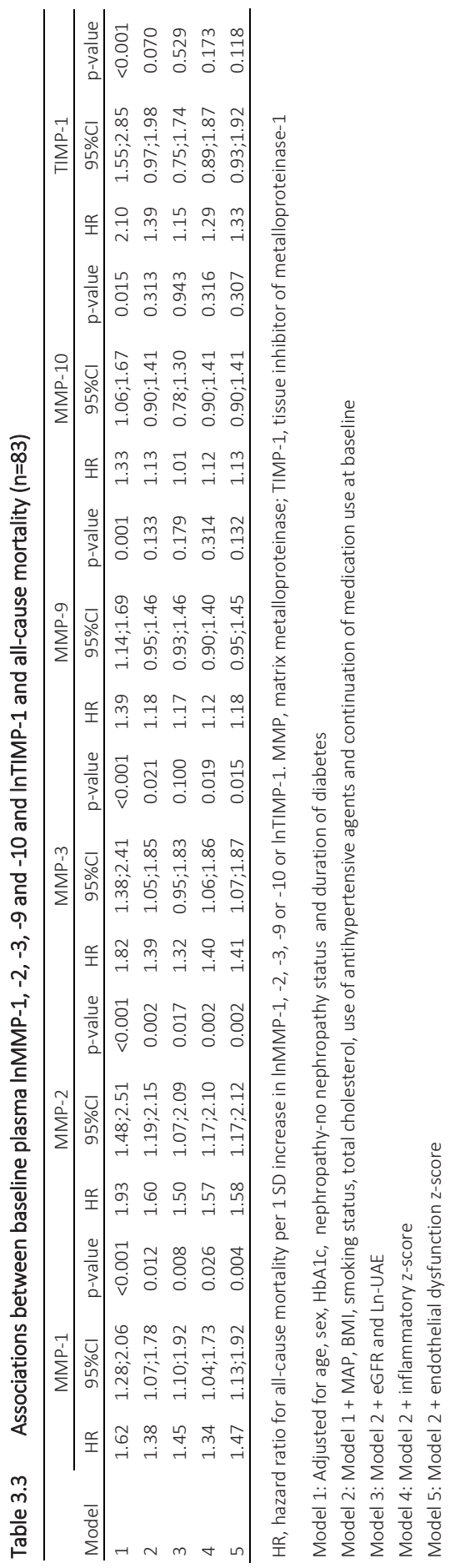




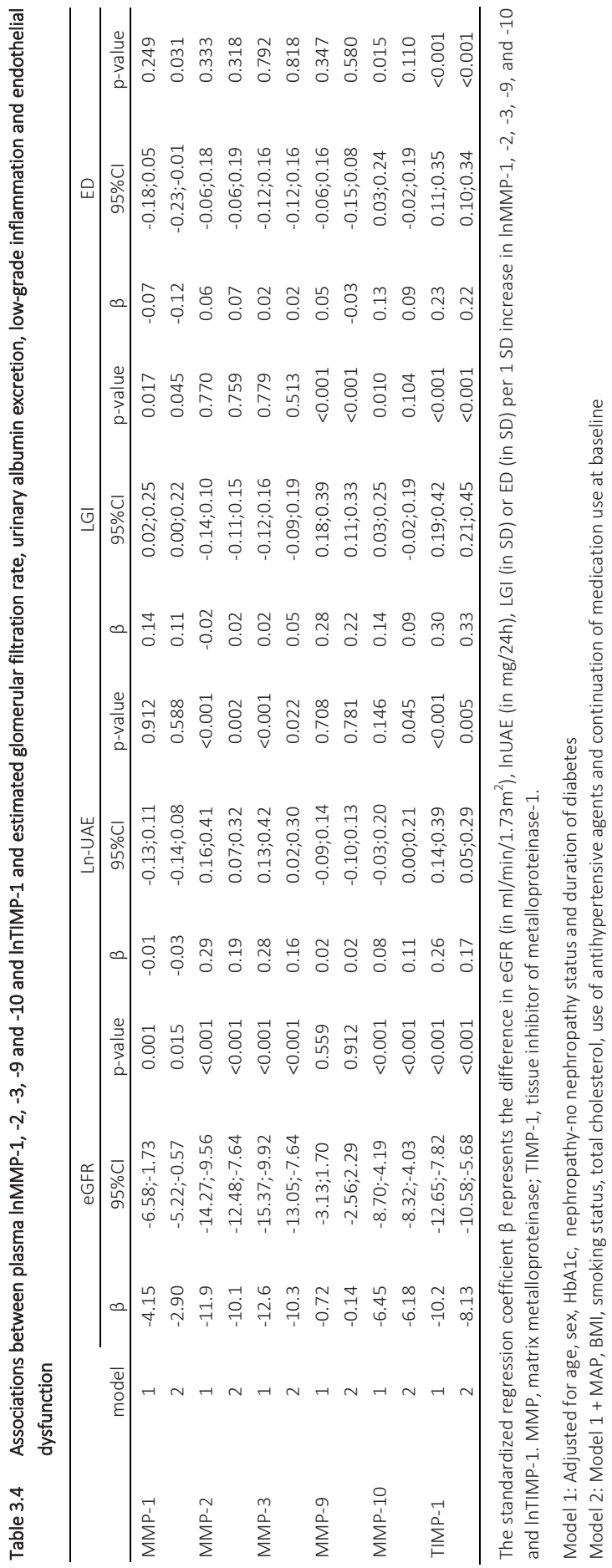




\section{MMP-2 and -3 and eGFR decline during follow-up}

The significant associations between InMMP-2 and incident CVD and between InMMP-3 and all-cause mortality were attenuated after adjustment for eGFR. To further investigate the role of eGFR in the association between plasma levels of MMPs and TIMP-1 on the one hand and incident CVD and all-cause mortality on the other, we investigated to what extent baseline MMPs and TIMP-1 levels were associated with changes in eGFR during follow-up. In addition, we investigated to what extent changes in eGFR (in addition to baseline eGFR) explained the associations between plasma markers and outcome measures when added to model 2. Follow-up data on eGFR, during followup measurements in $2003,{ }^{20}$ were available in 317/337 patients; 20 patients were excluded because they underwent kidney transplantation during follow-up and no data were available on eGFR pretransplantation $(n=14)$ or because no follow-up eGFR measurement was available $(n=6)$. Median follow-up duration was 9.9 years $[9.1 ; 10.5]$. The median decrease in eGFR was $-1.67 \mathrm{ml} / \mathrm{min} / 1.73 \mathrm{~m}^{2}$ per year (interquartile range 3.18 to -0.64). After adjustment for age, sex, duration of diabetes, HbA1c, smoking, MAP, BMI, total cholesterol, antihypertensive medication and eGFR at baseline, InMMP2 and -3 (per 1 SD increase) were associated with decline in eGFR of $-0.24(95 \% \mathrm{Cl}$ $0.60 ; 0.12)$ and of $-0.55(-0.94 ;-0.16) \mathrm{ml} / \mathrm{min} / 1.73 \mathrm{~m}^{2}$ per year, respectively. Supplemental Figure S3.4 shows the decrease in eGFR per year according to tertiles of all MMPs and TIMP-1 after adjustment for the potential confounders used in model 2 plus baseline eGFR. The decline in eGFR was significantly greater in patients with nephropathy compared to those without $(p<0.001)$.

In the 317 patients with available data on eGFR during follow-up, the HR for developing CVD per 1 SD higher InMMP-2 decreased from $1.60(1.17 ; 2.18)$ to $1.40(1.00 ; 1.96)$ after additional adjustment for baseline eGFR. Further adjustment for the decline in eGFR further decreased the HR to $1.36(0.96 ; 1.92)$. This indicates that MMP-2-associated decrease of eGFR may partly explain the association between plasma MMP-2 and incident CVD. Next, in these 317 patients, the HR for all-cause mortality per $1 \mathrm{SD}$ increase in InMMP-3 decreased from $2.04(1.45 ; 2.88)$ to $1.80(1.22 ; 2.65)$ after additional adjustment for baseline eGFR. Further adjustment for the decrease in eGFR did not materially change the HR $[1.79(1.22 ; 2.62)]$.

\section{Additional analyses}

Stratified analyses showed that, after full adjustment for cardiovascular risk factors (model 2), one SD higher InTIMP-1 was associated with an increase in all-cause mortality in patients without [HR $3.65(1.49 ; 8.92)]$, but not in those with nephropathy at baseline [HR1.15 (0.75;1.76)] ( $p$ for interaction was 0.009). In addition, after full adjustment for cardiovascular risk factors (model 2), one SD higher InMMP-2 was associated with an increased HR for all-cause mortality in men [HR $1.99(1.34 ; 2.95)]$, but not in women [0.85 $(0.49 ; 1.46)]$ ( $p$ for interaction was 0.025$)$. 
Associations between plasma InMMPs and InTIMP-1 on the one hand and eGFR, InUAE, LGI and ED on the other differed significantly between patients with and without nephropathy (Supplemental Table S3.3 and S3.4). The associations between plasma InMMPs and InTIMP-1 and eGFR and UAE were generally stronger in patients with diabetic nephropathy. Associations between markers of LGI and ED on the one hand and MMPs and TIMP-1 on the other did not differ consistently between patients with or without nephropathy.

\section{Discussion}

The present study in type 1 diabetic patients, with a median follow-up of 12.3 years, shows significant associations between higher levels of plasma MMP-2 and incident CVD. In addition, higher plasma levels of MMP-1, -2 and -3 were significantly associated with all-cause mortality. These associations were independent of cardiovascular risk factors, LGI and ED (as expressed by z-scores), but partly dependent on eGFR and changes in eGFR. Despite the widespread correlations between the various MMPs and TIMP-1 (Supplemental Table S3.2), we showed specific associations as mentioned previously, which will be further discussed both with regards to current literature, but also on possible pathophysiological mechanisms.

\section{MMPs, TIMP-1 and CVD}

Higher MMP-2-levels were independently associated with incident cardiovascular events, which is supported by the few studies available in mainly non-diabetic subjects. ${ }^{12,28,29}$ In patients with atrial fibrillation, higher baseline plasma MMP-2 levels were independently associated with cardiovascular events or death during a mean follow-up of 28 months. ${ }^{12}$ In a Mexican population, a significant association between the specific MMP-2-1575 A/G polymorphism and occurrence of myocardial infarction has been established. ${ }^{28}$ Additionally, in patients with ST-elevation myocardial infarction undergoing percutaneous coronary intervention, higher plasma MMP-2-levels at baseline were associated with increased myocardial infarct size and decreased left ventricular function after four months of follow-up. ${ }^{29}$

Various mechanisms may contribute to MMP-2-mediated macrovascular disease. We hypothesize that the occurrence of macrovascular disease can be enhanced by increased extracellular MMP-2 activity leading to plaque rupture, thrombus formation and vasoconstriction caused by MMP-2-mediated cleavage of vasoactive peptides. This hypothesis is supported by current literature. ${ }^{4,30}$ Additionally, altered MMP-2 activity may trigger cardiovascular pathology by vascular remodeling, i.e. increased migration of smooth muscle vascular cells to the intima, increased fibrosis and decrease of elastin 
content. ${ }^{3,31}$ Furthermore, MMP-2 leads to sustained thrombus formation by increased platelet activation, adhesion and aggregation. ${ }^{4}$

In our study plasma MMP-1, -3, -9, -10 and TIMP-1-levels were not associated with incident CVD, which is in line with several prospective studies in the general population $^{32-34}$ and in patients with rheumatoid arthritis. ${ }^{35}$ In contrast, one study in 1127 patients with stable and unstable angina showed significant associations between higher MMP-9 levels and cardiovascular mortality with a HR of $2.41(1.26 ; 4.60)$ in the upper quartile. ${ }^{13}$ The discrepancies with our study can possibly be explained by the differences in study population and by the fact that we used a combined endpoint for fatal and nonfatal CVD. For the combined endpoint of cardiovascular death and nonfatal myocardial infarction in the study by Blankenberg et al., ${ }^{13}$ the unadjusted HR was $1.30(1.11 ; 1.52$, $p<0.002$ ) with increasing MMP-9 quartiles, but it is not clear whether or not this remained significant after adjustment for potential confounders. In our study we did not show an association between TIMP-1-levels and occurrence of CVD. This is in contrast to a recent study which showed a significant association between plasma TIMP-1-levels and recurrent cardiovascular events in patients suffering from previous myocardial infarction or unstable angina 3 to 36 months before enrollment. ${ }^{14}$ This discrepancy might be explained by the difference in study populations, as in our study patients with prior CVD were excluded. In addition, in our study more adjustments for potential confounders were made compared with the latter two studies.

\section{MMPs, TIMP-1 and all-cause mortality}

Baseline MMP-1, -2 and -3-levels were significantly associated with all-cause mortality during follow-up. Stratification according to sex showed that the association between MMP-2 and all-cause mortality was restricted to men. However, the present study can neither explain this sex interaction nor can it exclude the possibility of a chance finding. Thus far, no prospective studies have been published regarding the association between plasma MMPs and all-cause mortality in type 1 diabetic patients. In different study populations higher circulating levels of MMP-1, ${ }^{15}$ MMP-2 $2^{12,16,17}$ and MMP-3 ${ }^{18,19}$ were strongly associated with all-cause mortality in patients with colorectal cancer, ${ }^{15}$ myocardial infarction ${ }^{16,18}$ or heart failure. ${ }^{17,19}$ MMP-1 is thought to be involved in cancer progression and invasion, ${ }^{15}$ whereas the mechanism by which MMP-3 contributed was not clarified. ${ }^{18,19}$

Plasma TIMP-1 levels did not show a significant association with all-cause mortality in the total study group and in patients with nephropathy at baseline. In contrast, plasma TIMP-1 was positively associated with all-cause mortality in patients without nephropathy at baseline. However, the observed interaction between TIMP-1 and nephropathy-status should be interpreted with caution as we cannot exclude the play of chance. Previous studies on the association between plasma TIMP-1 and mortality have been conflicting, both no ${ }^{36}$ and positive associations ${ }^{34,37,38}$ have been described. Positive associations between TIMP-1 and mortality were encountered in the general 
population, ${ }^{34}$ patients who underwent coronary angiography ${ }^{37}$ and in patients with heart failure. $^{38}$ These diverse results do not allow us to draw a solid conclusion on the association between TIMP-1 and all-cause mortality for all study populations. However, in type 1 diabetes, the present study is the first to report on this association.

Certain genetic polymorphisms coding for MMP-1 and MMP-3 have been associated with all-cause mortality. ${ }^{39}$ The MMP-1 2G/2G polymorphism has been associated with increased mortality in a three-year follow-up study in 99 hemodialysis patients and 133 matched controls, HR 2.96 (1.29-6.80). In this study, the MMP-3 6A/6A polymorphism was non-significantly associated with all-cause mortality, HR 3.01 (0.88-10.3) and combined homozygosity of MMP-1 and MMP-3 polymorphisms lead to a HR of 4.69 (1.72-12.8). It is unknown, however, how these polymorphisms influence MMP expression and/or activity and the underlying mechanism of this association thus remains to be further investigated.

\section{Potential mediation through eGFR}

The association between plasma MMP-2 and CVD was attenuated after adjustment for baseline eGFR, which explained $\sim 10 \%$ of the association. The HR decreased further after adjustment for decline in eGFR during follow-up. This may indicate that eGFR and decline in eGFR play a mediating role in the association between MMP-2 and CVD. In support, MMP-2 has been shown to induce complex alterations characterizing renal tubular epithelial-mesenchymal transformation leading to increased renal fibrosis, ${ }^{40}$ which could lead to decreased renal function. Higher plasma MMP-2 levels may also be the result of decreased renal function. However, MMP-2 levels are only minimally dependent on renal clearance due to the molecular weight of MMP-2, which is higher than that of albumin.

The association between MMP-3 and all-cause mortality was also attenuated by eGFR and this explained $\sim 5 \%$ of the association. Although MMP-3 was significantly associated with eGFR decline during follow-up, the association between MMP-3 and mortality did not materially change after adjustment for eGFR decline. This makes a mediating role for eGFR in the association between MMP-3 and all-cause mortality less likely. Taken together, these results show that increased plasma levels of MMP-2 and -3, possible due to, but to a greater extent independent of, decreased eGFR are positively associated with CVD and all-cause mortality, respectively.

\section{Limitations}

There are several limitations to our study. Firstly, plasma levels of MMP-1, -2, -3, -9, -10 and of TIMP-1 were only determined at baseline. Changes in plasma levels over time, revealing more details about outcome measures, are not known. Secondly, we do not know to what extent plasma levels reflect the local pathological situation at tissue level. Thirdly, we only determined five different MMPs and one of the four known TIMPs, 
considered to be the most important MMPs and TIMP to contribute to our outcome measures, but we cannot exclude that other MMPs and TIMPs are involved. Fourthly, the non-significant associations between the MMPS/TIMP-1 and outcome measures could be due to lack of statistical power, because of the relatively small number of events. Patients who died during follow-up were classified as cardiovascular unless another cause was established and this could have biased our results towards the null, resulting in underestimation of associations. Finally, the associations between MMPs and TIMP-1 with eGFR changes over time might be weakened by excluding those with renal transplantation during follow-up. Besides these limitations, the current study, with extensive adjustments for potential confounders and mediators, provides additional and consistent information on ECM remodeling by MMPs and TIMP-1 preceding incident CVD and all-cause mortality in a large cohort of patients with type 1 diabetes. These results are generalizable to normo- and macroalbuminuric type 1 diabetic patients. Although microalbuminuric patients were not included in the current study, we have no reason to expect different associations in this group.

\section{Conclusions}

In patients with type 1 diabetes followed for a median of 12 years, higher plasma MMP-2 levels are associated with incident CVD and higher plasma MMP-1, MMP-2, MMP-3 are associated with all-cause mortality. These associations are independent of cardiovascular risk factors, low-grade inflammation and endothelial dysfunction. However, baseline eGFR and decline in eGFR during follow-up attenuated the association between MMP-2 and CVD as well as the association between MMP-3 and allcause mortality; eGFR may thus, in part, mediate these associations. 


\section{References}

1. de Ferranti SD, de Boer IH, Fonseca V, Fox CS, Golden SH, Lavie CJ, Magge SN, Marx N, McGuire DK, Orchard TJ, Zinman B, Eckel RH: Type 1 Diabetes Mellitus and Cardiovascular Disease: A Scientific Statement From the American Heart Association and American Diabetes Association. Diabetes Care 2014;37:2843-2863

2. Peeters SA, Engelen L, Buijs J, Chaturvedi N, Fuller JH, Schalkwijk CG, Stehouwer CD, Group EPCS: Plasma levels of matrix metalloproteinase-2, $-3,-10$, and tissue inhibitor of metalloproteinase- 1 are associated with vascular complications in patients with type 1 diabetes: the EURODIAB Prospective Complications Study. Cardiovasc Diabetol 2015;14:31

3. Chen Q, Jin M, Yang F, Zhu J, Xiao Q, Zhang L: Matrix metalloproteinases: inflammatory regulators of cell behaviors in vascular formation and remodeling. Mediators Inflamm 2013;2013:928315

4. Gresele P, Falcinelli E, Loffredo F, Cimmino G, Corazzi T, Forte L, Guglielmini G, Momi S, Golino P: Platelets release matrix metalloproteinase-2 in the coronary circulation of patients with acute coronary syndromes: possible role in sustained platelet activation. Eur Heart J 2011;32:316-325

5. Visse R, Nagase H: Matrix metalloproteinases and tissue inhibitors of metalloproteinases: structure, function, and biochemistry. Circ Res 2003;92:827-839

6. Chase AJ, Newby AC: Regulation of matrix metalloproteinase (matrixin) genes in blood vessels: a multistep recruitment model for pathological remodelling. J Vasc Res 2003;40:329-343

7. Giebel SJ, Menicucci G, McGuire PG, Das A: Matrix metalloproteinases in early diabetic retinopathy and their role in alteration of the blood-retinal barrier. Lab Invest 2005;85:597-607

8. Ebihara I, Nakamura T, Shimada N, Koide H: Increased plasma metalloproteinase-9 concentrations precede development of microalbuminuria in non-insulin-dependent diabetes mellitus. Am J Kidney Dis 1998;32:544-550

9. Thrailkill KM, Bunn RC, Moreau CS, Cockrell GE, Simpson PM, Coleman HN, Frindik JP, Kemp SF, Fowlkes $\mathrm{JL}$ : Matrix metalloproteinase-2 dysregulation in type 1 diabetes. Diabetes Care 2007;30:2321-2326

10. Toni M, Hermida J, Goni MJ, Fernandez P, Parks WC, Toledo E, Montes R, Diez N: Matrix metalloproteinase-10 plays an active role in microvascular complications in type 1 diabetic patients. Diabetologia 2013;56:2743-2752

11. Jacqueminet S, Ben Abdesselam O, Chapman MJ, Nicolay N, Foglietti MJ, Grimaldi A, Beaudeux JL: Elevated circulating levels of matrix metalloproteinase- 9 in type 1 diabetic patients with and without retinopathy. Clin Chim Acta 2006;367:103-107

12. Ehrlich JR, Kaluzny M, Baumann S, Lehmann R, Hohnloser SH: Biomarkers of structural remodelling and endothelial dysfunction for prediction of cardiovascular events or death in patients with atrial fibrillation. Clin Res Cardiol 2011;100:1029-1036

13. Blankenberg S, Rupprecht HJ, Poirier O, Bickel C, Smieja M, Hafner G, Meyer J, Cambien F, Tiret L: Plasma concentrations and genetic variation of matrix metalloproteinase 9 and prognosis of patients with cardiovascular disease. Circulation 2003;107:1579-1585

14. West MJ, Nestel PJ, Kirby AC, Schnabel R, Sullivan D, Simes RJ, Pollicino C, Lubos E, Munzel TF, White HD, Tonkin AM, Bickel C, Tiret L, Blankenberg S: The value of N-terminal fragment of brain natriuretic peptide and tissue inhibitor of metalloproteinase-1 levels as predictors of cardiovascular outcome in the LIPID study. Eur Heart J 2008;29:923-931

15. Tahara K, Mimori K, linuma H, Iwatsuki M, Yokobori T, Ishii H, Anai H, Kitano S, Mori M: Serum matrixmetalloproteinase-1 is a bona fide prognostic marker for colorectal cancer. Ann Surg Oncol 2010;17:3362-3369

16. Dhillon OS, Khan SQ, Narayan HK, Ng KH, Mohammed N, Quinn PA, Squire IB, Davies JE, Ng LL: Matrix metalloproteinase-2 predicts mortality in patients with acute coronary syndrome. Clin Sci (Lond) 2010;118:249-257

17. George J, Patal S, Wexler D, Roth A, Sheps D, Keren G: Circulating matrix metalloproteinase-2 but not matrix metalloproteinase-3, matrix metalloproteinase-9, or tissue inhibitor of metalloproteinase-1 predicts outcome in patients with congestive heart failure. Am Heart J 2005;150:484-487 
18. Kelly D, Khan S, Cockerill G, Ng LL, Thompson M, Samani NJ, Squire IB: Circulating stromelysin-1 (MMP3): a novel predictor of LV dysfunction, remodelling and all-cause mortality after acute myocardial infarction. Eur J Heart Fail 2008;10:133-139

19. Narayan H, Ng L, Squire I, Mohammed N, Quinn P: 51 Plasma MMP-3: A Novel Indicator of Left Ventricle Remodelling and Adverse Outcomes in Patients with Acute Heart Failure. Heart 2014;100 Suppl 3:A29-30

20. Astrup AS, Tarnow L, Rossing P, Pietraszek L, Riis Hansen P, Parving HH: Improved prognosis in type 1 diabetic patients with nephropathy: a prospective follow-up study. Kidney Int 2005;68:1250-1257

21. Tarnow L, Cambien F, Rossing P, Nielsen FS, Hansen BV, Lecerf L, Poirier O, Danilov S, Parving HH: Lack of relationship between an insertion/deletion polymorphism in the angiotensin I-converting enzyme gene and diabetic nephropathy and proliferative retinopathy in IDDM patients. Diabetes 1995; 44:489-494

22. Levey AS, Stevens LA, Schmid CH, Zhang YL, Castro AF, 3rd, Feldman HI, Kusek JW, Eggers P, Van Lente F, Greene T, Coresh J: A new equation to estimate glomerular filtration rate. Ann Intern Med 2009;150:604-612

23. Carrillo S: Updated Information for IDMS Traceable VITROS ${ }^{\circledR}$ Chemistry Products CREA Slides. Ortho Clinical Diagnostics (OCD), Rochester, NY 2008;

24. De Jager J, Kooy A, Lehert P, Bets D, Wulffele MG, Teerlink T, Scheffer PG, Schalkwijk CG, Donker AJ, Stehouwer CD: Effects of short-term treatment with metformin on markers of endothelial function and inflammatory activity in type 2 diabetes mellitus: a randomized, placebo-controlled trial. J Intern Med 2005;257:100-109

25. Schram MT, Chaturvedi N, Schalkwijk CG, Fuller JH, Stehouwer CD, Group EPCS: Markers of inflammation are cross-sectionally associated with microvascular complications and cardiovascular disease in type 1 diabetes--the EURODIAB Prospective Complications Study. Diabetologia 2005;48:370-378

26. Nin JW, Jorsal A, Ferreira I, Schalkwijk CG, Prins MH, Parving HH, Tarnow L, Rossing P, Stehouwer CD: Higher plasma soluble Receptor for Advanced Glycation End Products (sRAGE) levels are associated with incident cardiovascular disease and all-cause mortality in type 1 diabetes: a 12-year follow-up study. Diabetes 2010;59:2027-2032

27. van Bussel BC, Henry RM, Ferreira I, van Greevenbroek MM, van der Kallen CJ, Twisk JW, Feskens EJ, Schalkwijk CG, Stehouwer CD: A healthy diet is associated with less endothelial dysfunction and less lowgrade inflammation over a 7-year period in adults at risk of cardiovascular disease. J Nutr 2015;145: 532-540

28. Perez-Hernandez N, Vargas-Alarcon G, Martinez-Rodriguez N, Martinez-Rios MA, Pena-Duque MA, PenaDiaz Ade L, Valente-Acosta B, Posadas-Romero C, Medina A, Rodriguez-Perez JM: The matrix metalloproteinase 2-1575 gene polymorphism is associated with the risk of developing myocardial infarction in Mexican patients. J Atheroscler Thromb 2012;19:718-727

29. Nilsson L, Hallen J, Atar D, Jonasson L, Swahn E: Early measurements of plasma matrix metalloproteinase-2 predict infarct size and ventricular dysfunction in ST-elevation myocardial infarction. Heart 2012;98:31-36

30. Berry E, Bosonea AM, Wang X, Fernandez-Patron C: Insights into the activity, differential expression, mutual regulation, and functions of matrix metalloproteinases and a disintegrin and metalloproteinases in hypertension and cardiac disease. J Vasc Res 2013;50:52-68

31. Lan TH, Huang XQ, Tan HM: Vascular fibrosis in atherosclerosis. Cardiovasc Pathol 2013;22:401-407

32. Jefferis BJ, Whincup P, Welsh P, Wannamethee G, Rumley A, Lennon L, Thomson A, Lawlor D, Carson C, Ebrahim S, Lowe G: Prospective study of matrix metalloproteinase-9 and risk of myocardial infarction and stroke in older men and women. Atherosclerosis 2010;208:557-563

33. Welsh P, Whincup PH, Papacosta O, Wannamethee SG, Lennon L, Thomson A, Rumley A, Lowe GD: Serum matrix metalloproteinase- 9 and coronary heart disease: a prospective study in middle-aged men. Qjm 2008;101:785-791

34. Velagaleti RS, Gona P, Sundstrom J, Larson MG, Siwik D, Colucci WS, Benjamin EJ, Vasan RS: Relations of biomarkers of extracellular matrix remodeling to incident cardiovascular events and mortality. Arterioscler Thromb Vasc Biol 2010;30:2283-2288

35. Vuilleumier N, Bas S, Pagano S, Montecucco F, Guerne PA, Finckh A, Lovis C, Mach F, Hochstrasser D, Roux-Lombard P, Gabay C: Anti-apolipoprotein A-1 IgG predicts major cardiovascular events in patients with rheumatoid arthritis. Arthritis Rheum 2010;62:2640-2650 
36. Hansson J, Vasan RS, Arnlov J, Ingelsson E, Lind L, Larsson A, Michaelsson K, Sundstrom J: Biomarkers of extracellular matrix metabolism (MMP-9 and TIMP-1) and risk of stroke, myocardial infarction, and cause-specific mortality: cohort study. PLoS One 2011;6:e16185

37. Cavusoglu E, Ruwende C, Chopra V, Yanamadala S, Eng C, Clark LT, Pinsky DJ, Marmur JD: Tissue inhibitor of metalloproteinase-1 (TIMP-1) is an independent predictor of all-cause mortality, cardiac mortality, and myocardial infarction. Am Heart J 2006;151:1101 e1101-1108

38. Frantz S, Stork S, Michels K, Eigenthaler M, Ertl G, Bauersachs J, Angermann CE: Tissue inhibitor of metalloproteinases levels in patients with chronic heart failure: an independent predictor of mortality. Eur J Heart Fail 2008;10:388-395

39. Cozzolino M, Biondi ML, Galassi A, Turri O, Brancaccio D, Gallieni M: Matrix metalloproteinase-1 and matrix metalloproteinase-3 gene promoter polymorphisms are associated with mortality in haemodialysis patients. Nephrol Dial Transplant 2009;24:2207-2212

40. Cheng S, Lovett DH: Gelatinase A (MMP-2) is necessary and sufficient for renal tubular cell epithelialmesenchymal transformation. Am J Pathol 2003;162:1937-1949 


\section{Supplemental data}

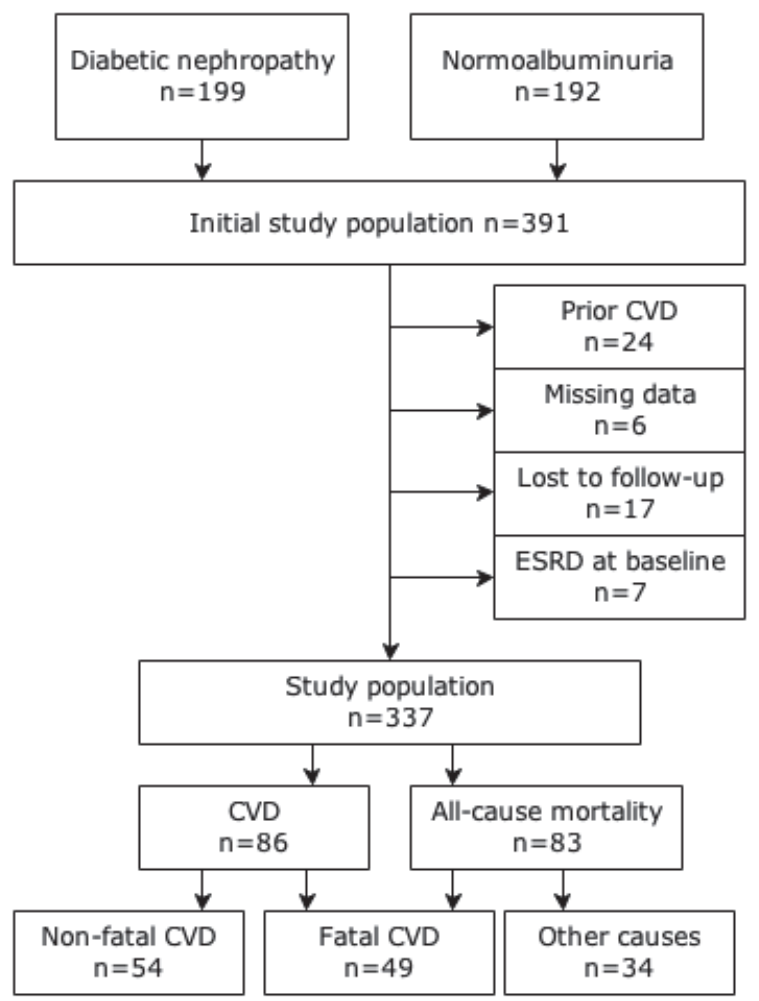

Figure S3.1 Overview of study population selection

Some patients suffered from a non-fatal as well as a fatal CVD event. The first event was used in the analyses. Cardiovascular disease (CVD), End stage renal disease (ESRD) 


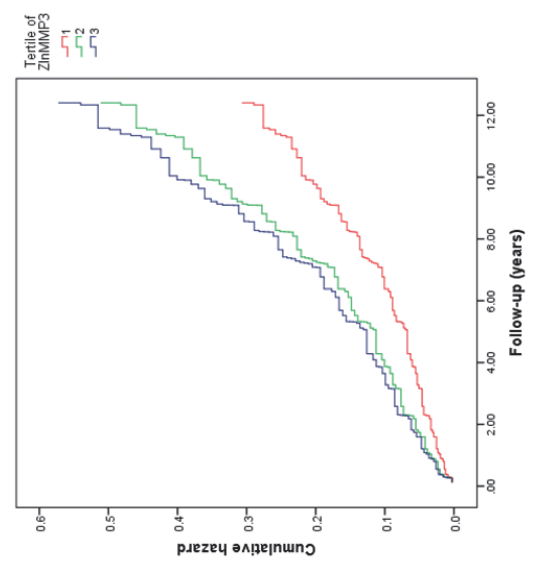

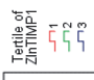

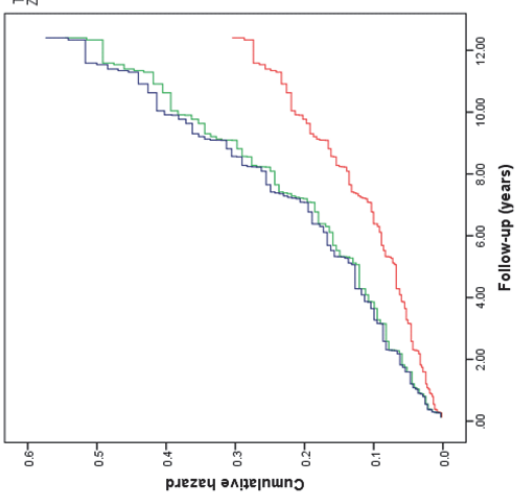

籍非

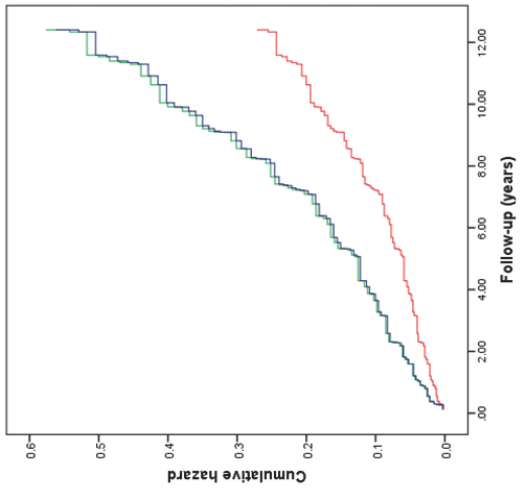

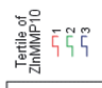

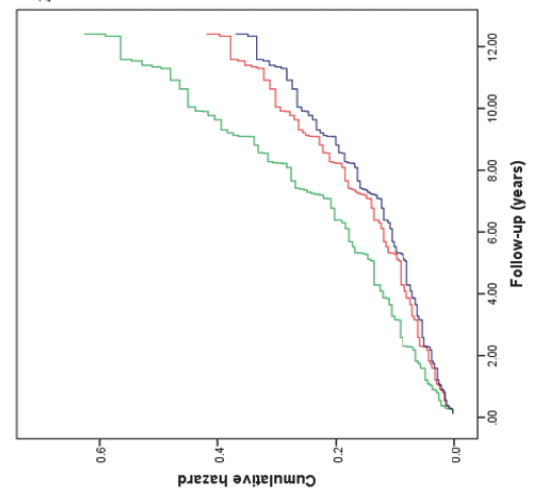

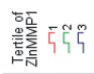
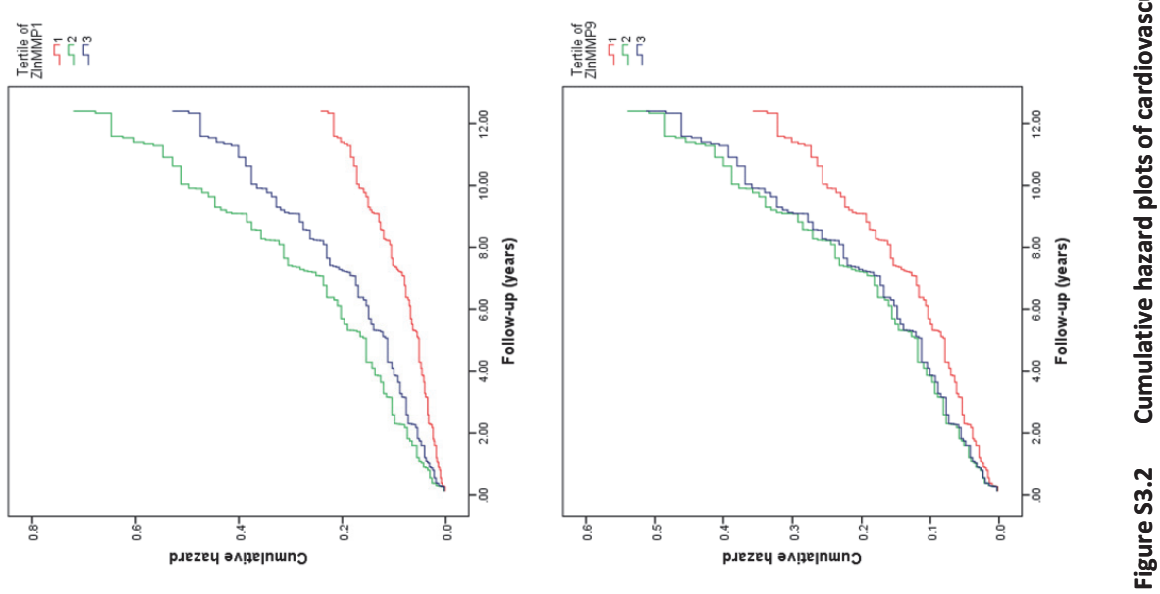

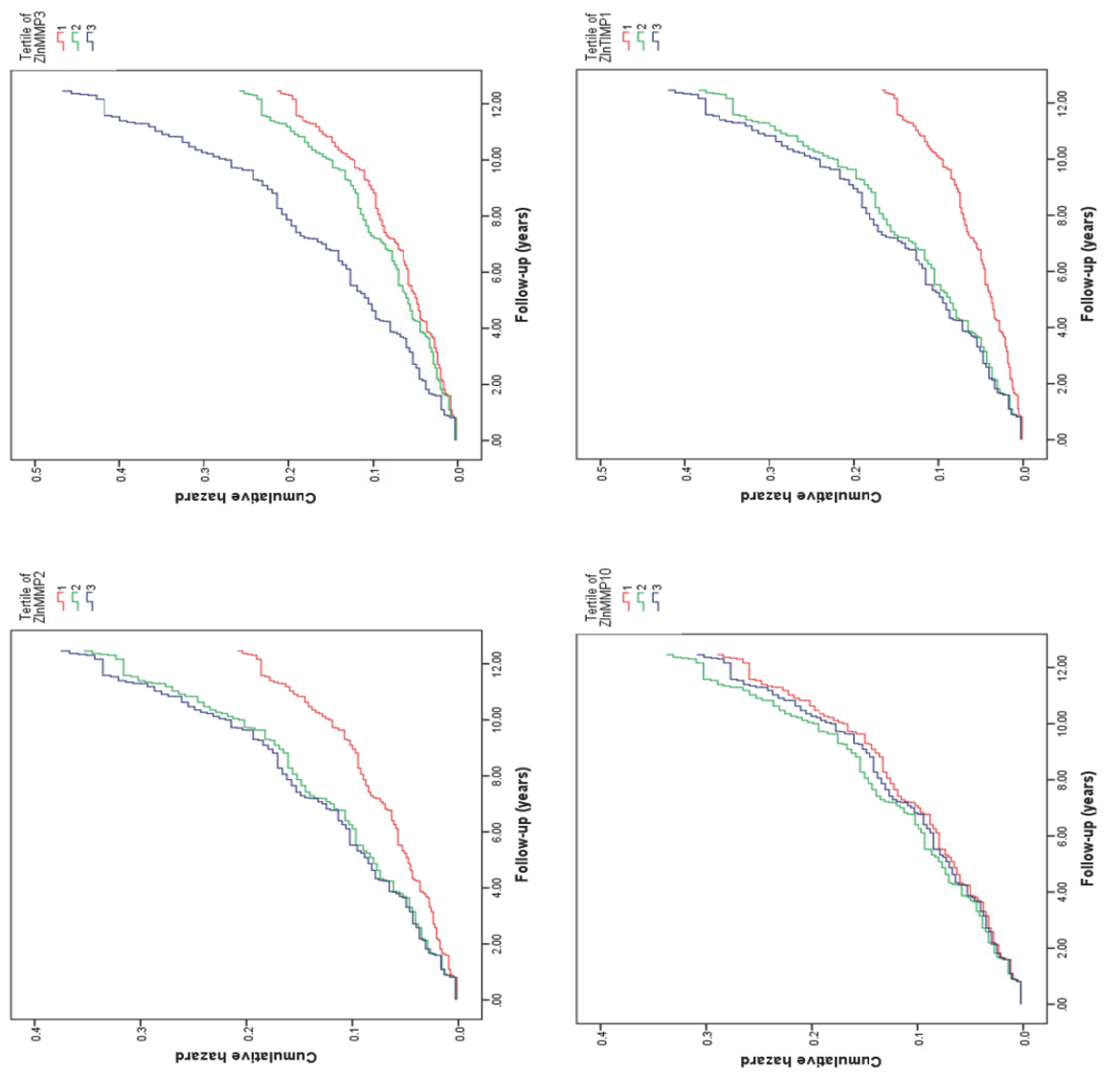

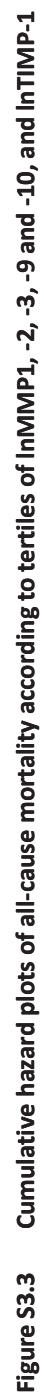



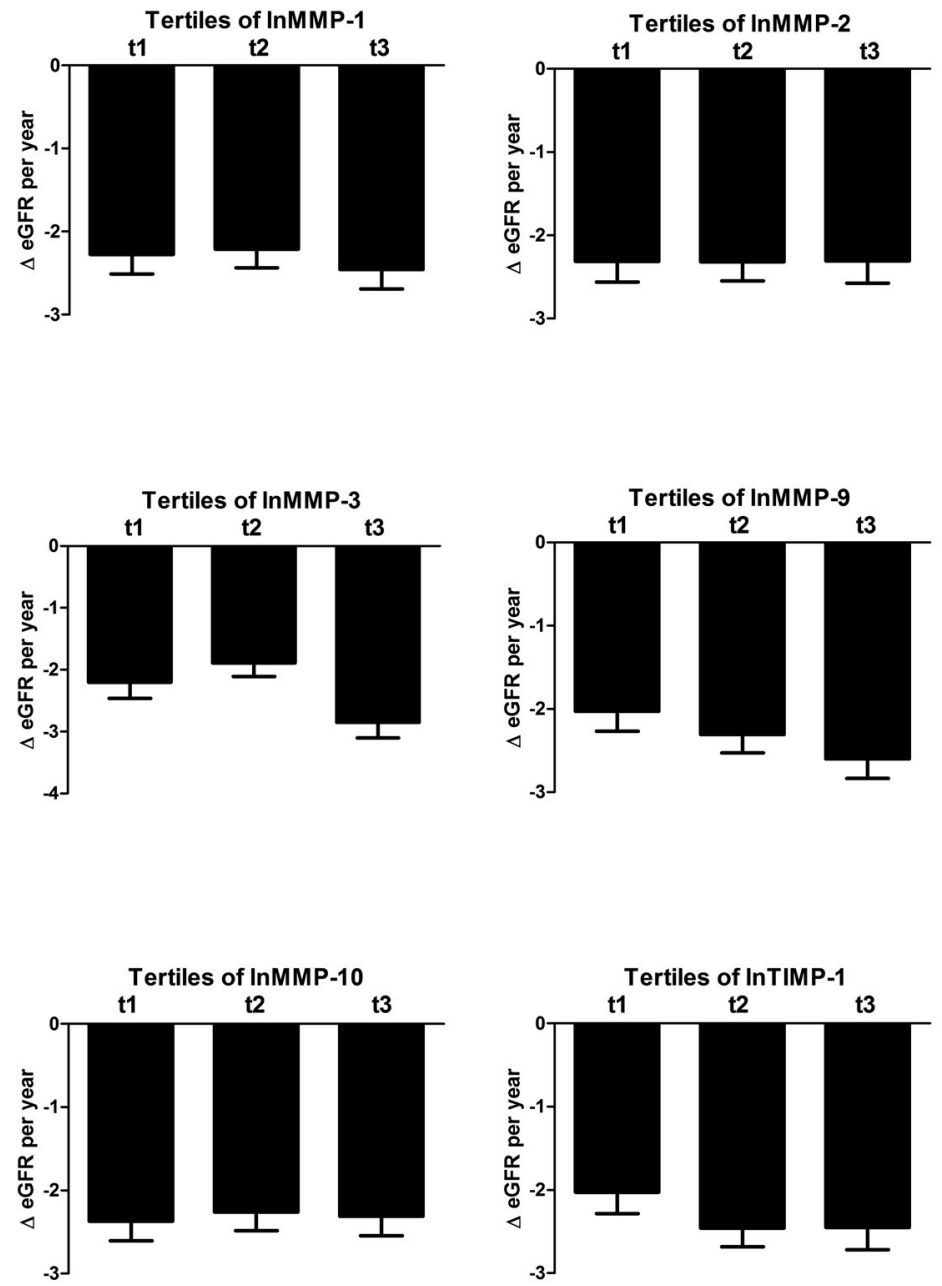

Figure S3.4 Decrease in eGFR $\left(\mathrm{ml} / \mathrm{min} / 1.73 \mathrm{~m}^{2}\right)$ per year according to tertiles of MMPs and TIMP-1 adjusted for sex, age, duration of diabetes, HbA1c, nephropathy-no nephropathy status, BMI, MAP, smoking status, antihypertensive treatment, continuation of antihypertensive treatment at baseline and baseline eGFR 
Table S3.1 Baseline characteristics according to nephropathy-no nephropathy status

\begin{tabular}{|c|c|c|c|}
\hline & $\begin{array}{c}\text { Nephropathy } \\
n=170\end{array}$ & $\begin{array}{c}\text { No nephropathy } \\
n=167\end{array}$ & p-value \\
\hline Age (yrs) & $40.4(9,5)$ & $42.6(9.7)$ & 0.035 \\
\hline Sex: male/female (\%) & $62 / 38$ & $59 / 41$ & 0.641 \\
\hline Duration of diabetes (yrs) & $27.9(8.1)$ & $27.6(8.0)$ & 0.690 \\
\hline $\mathrm{HbA} 1 \mathrm{c}(\%)$ & $9.5(1.5)$ & $8.5(1.1)$ & $<0.001$ \\
\hline $\mathrm{HbA} 1 \mathrm{c}(\mathrm{mmol} / \mathrm{mol})$ & $80.5(16.9)$ & $69.8(12.1)$ & $<0.001$ \\
\hline Retinopathy (no/simplex/proliferative) (\%) & $0 / 32 / 68$ & $35 / 54 / 11$ & $<0.001$ \\
\hline $\mathrm{BMI}\left(\mathrm{kg} / \mathrm{m}^{2}\right)$ & $24.1(3.3)$ & $23.7(2.5)$ & 0.187 \\
\hline LDL (mmol/l) & $3.48(1.03)$ & $2.84(0.87)$ & $<0.001$ \\
\hline $\mathrm{HDL}(\mathrm{mmol} / \mathrm{l})$ & $1.48(0.55)$ & $1.54(0.43)$ & 0.264 \\
\hline Triglycerides (mmol/l) & $1.21[0.85-1.66]$ & $0.77[0.57-0.95]$ & $<0.001$ \\
\hline Serum creatinine ( $\mu \mathrm{mol} / \mathrm{l})$ & $98.5[80-128]$ & $76[69-83]$ & $<0.001$ \\
\hline eGFR $\left(\mathrm{ml} / \mathrm{min} / 1.73 \mathrm{~m}^{2}\right)$ & $72.4(28.5)$ & $97.3(13.3)$ & $<0.001$ \\
\hline Urinary albumin excretion (mg/24h) & 823 [360-2050] & $8[5-13]$ & $<0.001$ \\
\hline Systolic blood pressure (mmHg) & $151(23)$ & $132(18)$ & $<0.001$ \\
\hline Diastolic blood pressure ( $\mathrm{mmHg}$ ) & $86(12)$ & $76(10)$ & $<0.001$ \\
\hline Mean arterial pressure (mmHg) & $108(14)$ & $94(11)$ & $<0.001$ \\
\hline RAAS-inhibitors (\%) & 54 & 4 & $<0.001$ \\
\hline Other antihypertensive medication (\%) & 66 & 9 & $<0.001$ \\
\hline Smoking (never/former/current) (\%) & $32 / 17 / 51$ & $39 / 19 / 42$ & 0.262 \\
\hline MMP-1 (ng/ml) & $4.08[2.51-6.89]$ & $2.21[1.43-3.49]$ & $<0.001$ \\
\hline MMP-2 (ng/ml) & $230[194-266]$ & $180[156-200]$ & $<0.001$ \\
\hline MMP-3 (ng/ml) & $20.1[12.6-28.7]$ & $10.6[7.8-16.6]$ & $<0.001$ \\
\hline MMP-9 (ng/ml) & $31.1[21.0-56.0]$ & $18.5[13.2-30.5]$ & $<0.001$ \\
\hline MMP-10 (pg/ml) & 785 [525-1093] & 612 [443-873] & 0.002 \\
\hline TIMP-1 (ng/ml) & 216 [174-264] & $149[129-174]$ & $<0.001$ \\
\hline sVCAM-1 (ng/ml) & 1018 [860-1204] & $880[759-994]$ & $<0.001$ \\
\hline slCAM-1 (ng/ml) & $711[586-848]$ & $672[574-776]$ & 0.063 \\
\hline Endothelial dysfunction z-score & $0.23(0.82)$ & $-0.23(0.67)$ & $<0.001$ \\
\hline $\mathrm{CRP}(\mathrm{mg} / \mathrm{l})$ & $1.22[0.59-2.94]$ & $0.92[0.34-2.06]$ & 0.720 \\
\hline $\mathrm{IL}-6$ (pg/ml) & $2.06[1.25-3.38]$ & $1.40[0.93-2.08]$ & 0.743 \\
\hline $\mathrm{sPLA} 2(\mu \mathrm{g} / \mathrm{ml})$ & $4.40[2.70-6.73]$ & $4.00[2.80-6.10]$ & 0.441 \\
\hline Low-grade inflammation z-score & $0.13(0.64)$ & $-0.14(0.67)$ & $<0.001$ \\
\hline
\end{tabular}

Data are means (SD), medians [inter-quartile range] or percentages, as appropriate. eGFR, estimated glomerular filtration rate by Chronic Kidney Disease Epidemiology Collaboration (CKD-EPI); RAAS-inhibitors, renin-angiotensin-aldosterone system inhibitors, including angiotensin converting enzyme inhibitors, angiotensin II receptor blockers and spironolactone; MMP, matrix metalloproteinase; TIMP-1, tissue inhibitor of metalloproteinase-1; sVCAM-1, soluble vascular cell adhesion molecule-1; sICAM-1, soluble intracellular adhesion molecule-1; CRP, C-reactive protein; IL-6, interleukin-6; sPLA2, secreted phospholipase A2; Low-grade inflammation z-score, z-score of the average of the z-scores of InIL-6, InCRP, sICAM-1, and InsPLA2; Endothelial dysfunction z-score, z-score of the average of the z-scores of sICAM-1 and SVCAM-1 
Table S3.2 Correlations between plasma MMP-1, $-2,-3,-9$ and -10 , and TIMP-1 ( $=337)$

\begin{tabular}{|c|c|c|c|c|c|c|c|c|c|c|c|c|}
\hline & \multicolumn{2}{|c|}{ MMP-1 } & \multicolumn{2}{|c|}{ MMP-2 } & \multicolumn{2}{|c|}{ MMP-3 } & \multicolumn{2}{|c|}{ MMP-9 } & \multicolumn{2}{|c|}{ MMP-10 } & \multicolumn{2}{|c|}{ TIMP-1 } \\
\hline & $r$ & $p$-value & $r$ & $p$-value & $r$ & $p$-value & $r$ & $p$-value & $r$ & $p$-value & $r$ & $p$-value \\
\hline MMP-1 & 1.00 & - & 0.35 & $<0.001$ & 0.33 & $<0.001$ & 0.28 & $<0.001$ & 0.11 & 0.05 & 0.51 & $<0.001$ \\
\hline MMP-2 & .35 & $<0.001$ & 1.00 & - & 0.53 & $<0.001$ & 0.05 & & 0.28 & $<0.001$ & 0.62 & $<0.001$ \\
\hline & 0.33 & $<0.001$ & 0.53 & $<0.001$ & & - & & & & & 0.55 & \\
\hline MMP-9 & 0.28 & $<0.001$ & 0.05 & 0.346 & 0.08 & 16 & 1.00 & - & 0.07 & 0.197 & 0.25 & $<0.001$ \\
\hline MMP-10 & 0.11 & 0.05 & 0.28 & $<0.001$ & 0.31 & $<0.001$ & 0.07 & 0.197 & 1.00 & - & 0.29 & $<0.001$ \\
\hline TIMP-1 & 0.51 & $<0.001$ & 0.62 & $<0.001$ & 0.55 & $<0.001$ & 0.25 & $<0.001$ & 0.29 & $<0.001$ & 1.00 & - \\
\hline
\end{tabular}

$r$, Pearson correlation 


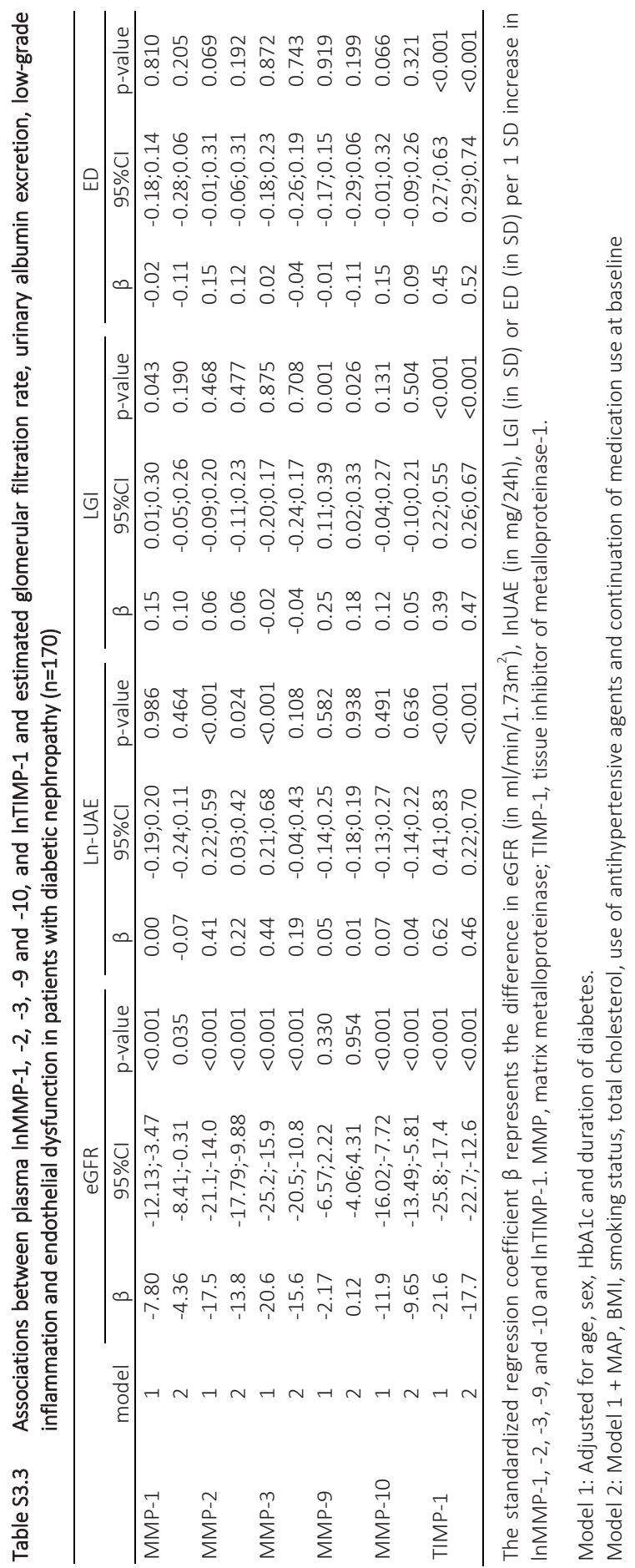




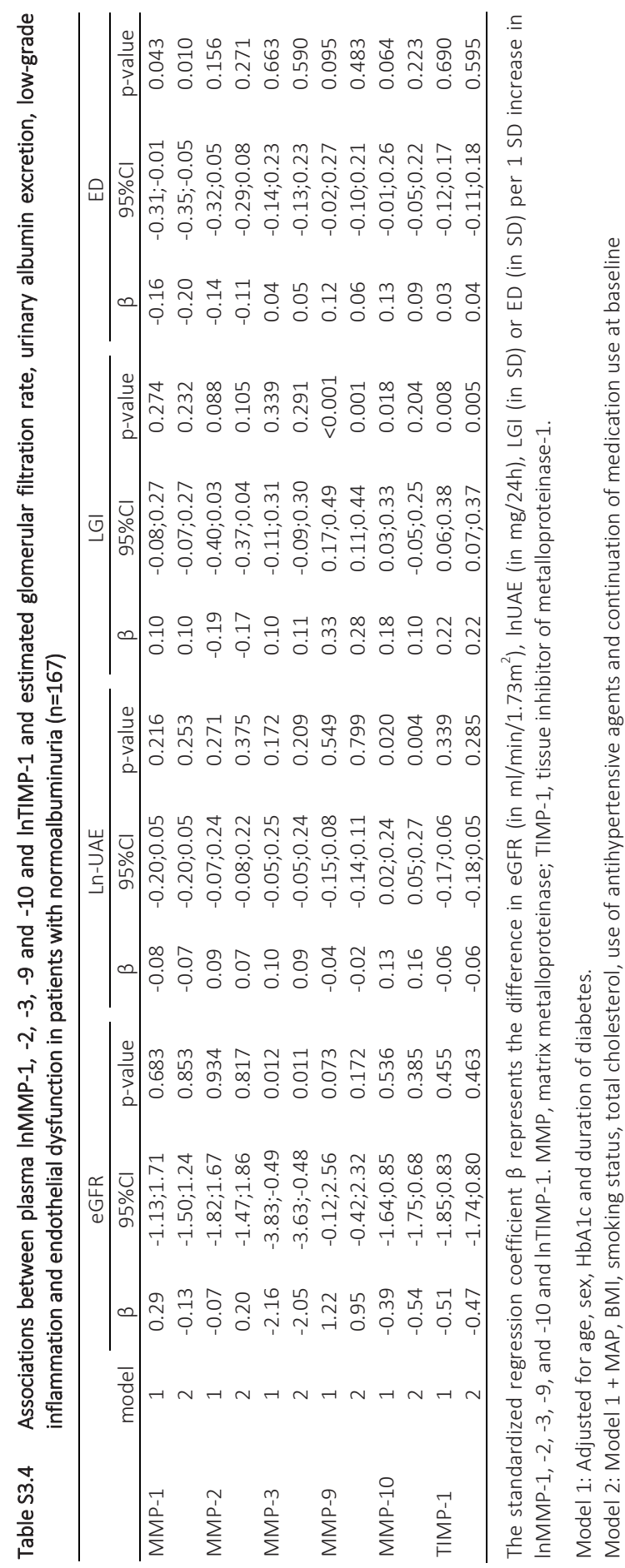





\section{Chapter 4}

Circulating matrix metalloproteinases are associated with arterial stiffness in patients with type 1 diabetes: pooled analysis of three cohort studies

S.A. Peeters, L. Engelen, J. Buijs, N. Chaturvedi, J.H. Fuller, A. Jorsal, H.-H. Parving, L. Tarnow, S. Theilade, P. Rossing, C.G. Schalkwijk, C.D.A. Stehouwer Accepted for publication in Cardiovasc Diabetol. 


\section{Abstract}

\section{Objective}

Altered regulation of extracellular matrix (ECM) composition by matrix metalloproteinases (MMPs) and tissue inhibitors of metalloproteinase (TIMPs) may contribute to arterial stiffening. We investigated associations between circulating MMP1, - $2,-3,-9,-10$ and TIMP-1, and carotid-femoral pulse wave velocity (cfPWV) and pulse pressure (PP), as markers of arterial stiffness in type 1 diabetic patients.

\section{Methods}

Individuals with type 1 diabetes from three different cohorts were included in this study: EURODIAB Prospective Complications study ( $n=509)$, LEACE $(n=370)$ and PROFIL $(n=638)$. Linear regression analyses were used to investigate cross-sectional associations between circulating levels of MMP-1, $-2,-3,-9,-10$, and TIMP-1 and cfPWV ( $n=614)$ as well as office PP ( $n=1517)$. Data on 24-h brachial and 24-h central PP were available in 638 individuals from PROFIL. Analyses were adjusted for age, sex, duration of diabetes, $\mathrm{HbA1c}$, mean arterial pressure (MAP), and eGFR, and additionally for other cardiovascular risk factors and presence of vascular complications.

\section{Results}

After adjustment for potential confounders and presence of vascular complications, circulating MMP-3 was associated with cfPWV [ $\beta$ per 1 SD higher InMMP3 0.29 m/s (0.02;0.55)]. In addition, brachial and central 24-h PP measurements in PROFIL were significantly associated with MMP-2 ((1.40(0.47:2.33) and 1.43(0.63:2.23)). Pooled data analysis showed significant associations of circulating levels of MMP-1 and MMP-2 with office PP [ $\beta$ per 1 SD higher InMMP-1 and InMMP-2 = -0.83 mmHg (95\% Cl: $-1.50 ;-0.16)$ and $=1.33 \mathrm{mmHg}(0.55 ; 2.10)$, respectively].

\section{Conclusions}

MMPs-1, -2 , and -3 are independently associated with markers of arterial stiffening in patients with type 1 diabetes and may become therapeutic targets. 


\section{Introduction}

Arterial stiffening is an important pathway through which diabetes causes cardiovascular disease. ${ }^{1}$ The current gold standard to quantify arterial stiffness ${ }^{2}$ is carotid-femoral pulse wave velocity (cfPWV) measurements, which have shown to be significantly higher in type 1 diabetic patients with previous CVD compared to patients without CVD after multivariable adjustment. ${ }^{3}$ In addition, previous studies in individuals with type 1 diabetes have shown independent associations between higher pulse pressure (PP), another and older marker of arterial stiffness, and incident cardiovascular disease $(\mathrm{CVD})^{4,5}$ and all-cause mortality. ${ }^{4}$

Although the pathogenic mechanism leading to greater arterial stiffness in type 1 diabetes has not been fully elucidated, extracellular matrix (ECM) remodeling, by matrix metalloproteinases (MMPs) and tissue inhibitor of metalloproteinases (TIMPs), is thought to contribute to alterations in vascular collagen and/or elastin content, proliferation and migration of vascular smooth muscle cells, and plaque instability, potentially leading to greater arterial stiffness, hypertension and subsequent micro- and macrovascular complications. ${ }^{6-8}$

So far, studies reporting on associations between circulating levels of MMPs and TIMP on the one hand and arterial stiffness on the other have been inconsistent. cfPWV has been positively associated with (pro)MMP-1, ${ }^{9-11}$ MMP-2, ${ }^{12-14}$ MMP-9, ${ }^{12}$ and TIMP-1, ${ }^{15}$ whereas null associations between circulating levels of MMP-9 $9^{13,15,16}$ and TIMP-1 $1^{13,16}$ and cfPWV have also been published. Only one studies showed a positive association between MMP-2 and PP. ${ }^{17}$ Most studies were relatively small and addressed only a limited number of MMPs, and none focused specifically on individuals with type 1 diabetes.

Therefore, the aim of our study was to investigate associations between circulating levels of MMP-1, $-2,-3,-9$, and -10 and of TIMP-1 with markers of arterial stiffness, i.e. cfPWV and PP, in individuals with type 1 diabetes.

\section{Methods}

\section{Study population}

To study possible associations between circulating markers of ECM remodeling and cfPWV, the current gold standard for arterial stiffness, we used the PROFIL study, ${ }^{3}$ which is a cross-sectional study that was conducted at the Steno Diabetes Center in Denmark, between 2009 and 2011, and included 676 individuals with type 1 diabetes. In the diabetes group, 316 patients were normoalbuminuric, 169 had microalbuminuria and 191 had macroalbuminuria (by design). 
Further evaluation of arterial stiffness in individuals with type 1 diabetes, as expressed by PP, was performed by combining data from the PROFIL study with data from the EURODIAB Prospective Complications Study ${ }^{18-20}$ and the LEACE study. ${ }^{21}$ We added these analyses in multiple studies to investigate external validity and reproducibility, as cfPWV was only present in 1 study. In brief, the cross-sectional EURODIAB nested case-control study, which was performed as follow-up from the EURODIAB Prospective Complications Study, included 543 individuals with type 1 diabetes. Selected cases $(n=348)$ were those with one or more vascular complications, whereas controls $(n=195)$ were those without any evidence of vascular complications. The LEACE study is a prospective cohort study conducted at the Steno Diabetes Center in Denmark, with baseline measurements performed in 1993 when 391 individuals with type 1 diabetes were included. In this study group, 199 had diabetic nephropathy and 192 had normoalbuminuria (by design). Individuals with missing data on MMPs or TIMP-1 $(n=25)$, office PP $(n=15)$ or potential confounders $(n=53)$ were excluded from the present analyses, resulting in a total study population of 1,517 individuals (509 from EURODIAB, 370 from LEACE and 638 from PROFIL). The local Ethics Committees approved the studies and all patients gave informed consent.

\section{Main determinants}

Concentrations of MMP-1, MMP-2, MMP-3, MMP-9, MMP-10 and TIMP-1 were determined in EDTA plasma (for LEACE) or serum samples (for EURODIAB and PROFIL) that were stored at $-80^{\circ} \mathrm{C}$ until analyses. Circulating levels of MMPs and TIMP-1 were measured using a commercially available multi-array enzyme-linked immunosorbent assay (ELISA) kit [Human MMP-3-Plex Kit (for MMP-1, -3 and -9), Human MMP-2-Plex Kit (for MMP-2 and -10) and Human TIMP-1 Kit, MSD, Rockville, United States of America] according to the manufacturer's protocol. The MMPs were detected in both a pro- and an active form. TIMP-1 was detected only in the active form. The intra- and inter-assay coefficients of variation were $<10 \%$ for MMP-1, $-2,-3,-9$, and -10 and TIMP-1 in all studies, except for the inter-assay coefficient of variation of MMP-1 in PROFIL (10.4\%), the inter-assay coefficients of variation in all studies for MMP-3 (10.8 - 14.1\%) and the inter-assay coefficient of variation for MMP-9 in LEACE (13.7\%).

\section{Main outcomes}

cfPWV and/or PP were studied as markers of arterial stiffness. cfPWV measurements were performed in the PROFIL study and were available in 614 of the 638 participants. Trained laboratory technicians performed the measurements with the SphygmoCor device (AtCor Medical, Sydney, Australia) in participants in a supine position after a resting period of 15 minutes. Three cfPWV measurements were recorded and the two measurements closest to each other were averaged and used in the analyses. 
In the EURODIAB and the LEACE studies, a random zero sphygmomanometer (Hawskley, Lancing, UK) with the appropriate cuff size was used to measure arterial blood pressure. In the EURODIAB study, measurements were performed in a seated position after resting for 5 minutes, ${ }^{22}$ whereas in the LEACE study measurements were performed after at least 10 minutes of rest in supine position. ${ }^{23}$ In the PROFIL study, brachial blood pressure was determined after resting for 15 minutes in supine position (A\&D Medical, Tokyo, Japan) using an appropriate cuff size. ${ }^{24} \mathrm{PP}(\mathrm{mmHg})$ was calculated by subtracting diastolic blood pressure from systolic blood pressure. Besides PP, in 638 patients from the PROFIL study, data on 24-hour ambulatory blood pressure measurements (ABPM) were available. A validated tonometric watch-like device (BPro, HealthStats, Singapore) was used to record these 24-h ABPM. This device captured radial pulse wave reflections and calculated ABPM. ${ }^{25,26}$ The device was calibrated to brachial blood pressure and calculated central blood pressure. Blood pressure was measured every 15 minutes. ABPM data were considered adequate when at least 14 and 7 measurements were performed during the day (07.00 a.m. - 11.00 p.m.) and night (11.00 p.m.- 07.00 a.m.), respectively, according to current guidelines. ${ }^{27} \mathrm{~A}$ mean \pm SD of $45 \pm 7$ successful measurements were recorded during the day and $18 \pm 4$ during the night.

\section{Potential confounders}

Demographic data, information about medication, smoking history, duration of diabetes and use of antihypertensive medication were collected using questionnaires and patient records. Mean arterial pressure (MAP) was calculated as [systolic blood pressure + $(2 *$ diastolic blood pressure)]/3. Body weight and height were measured to calculate the body mass index (BMI). Blood samples were taken for measurements of total cholesterol, glycated hemoglobin ( $\mathrm{HbA} 1 \mathrm{c})$ and creatinine. We estimated the glomerular filtration rate (eGFR) using the Chronic Kidney Disease Epidemiology Collaboration (CKDEPI) equation. ${ }^{28}$

\section{Vascular complications}

Vascular complications were defined as presence of macro- and/or microvascular complications, i.e. CVD, albuminuria or retinopathy. CVD was defined, in all studies, as a cardiovascular event in a patient's medical history, including myocardial infarction, coronary intervention or stroke. ${ }^{3,21,29}$ In the EURODIAB study, angina and ischemic changes on ECG were also included in the definition of CVD. ${ }^{29}$ In addition, (intervention for) peripheral arterial disease was additionally included in LEACE and PROFIL ${ }^{3,21}$ Albumin excretion rates were measured in 24-hour urine collections. Microalbuminuria was defined as albumin excretion rate (AER) between 20 and $200 \mu \mathrm{g} / \mathrm{min}$ or between 30 and $300 \mathrm{mg} / 24 \mathrm{~h}$. Macroalbuminuria was defined as AER above $200 \mu \mathrm{g} / \mathrm{min}$ or $300 \mathrm{mg} / 24 \mathrm{~h}$. 
Retinopathy was assessed by retinal photography in all studies. Individuals were classified as no retinopathy, non-proliferative retinopathy, or proliferative retinopathy. In the PROFIL study blindness was also classified. Classification was conducted according to local protocols. ${ }^{3,21,30}$

\section{Statistical analyses}

All analyses were performed using the Statistical Package for Social Sciences (SPSS), version 20 (IBM Corporation, Armonk, NY, USA). Log-transformation was performed for variables with a skewed distribution (i.e. MMP-1, MMP-2, MMP-3, MMP-9, MMP-10 and TIMP-1).

One-way ANOVA and Chi-square tests were performed for comparison of baseline characteristics between the tertiles of cfPWV and of office PP. Linear regression analyses were applied to examine associations between circulating levels of MMP-1, MMP-2, MMP-3, MMP-9, MMP-10 and TIMP-1 on the one hand and cfPWV $(n=614)$, office PP $(n=1517)$, or 24-h PP $(n=638)$ measurements on the other. Analyses of these associations were adjusted for age, sex, duration of diabetes, HbA1c, eGFR, MAP, cohort and double inclusion (model 1). One hundred and thirty four patients were included in both the LEACE and the PROFIL study. However, inclusion in PROFIL was more than 15 years later than in LEACE. Therefore we decided to adjust for this instead of deleting these cases, which could lead to selection bias. The latter two adjustments in model 1 were only performed in case of office PP analysis. Further adjustments were made for total cholesterol, BMI, smoking status, use of antihypertensive medication and presence of any vascular complication (model 2). Results of these analyses are presented as $\mathrm{m} / \mathrm{s}$ higher cfPWV or mmHg higher PP per 1 SD higher level of circulating InMMP or InTIMP1. For this purpose, the MMP and TIMP-1 Z-scores were calculated cohort-specifically as absolute values may not be comparable due to sample preparation (i.e., plasma in LEACE vs. serum in EURODIAB and PROFIL). Furthermore, analyses of the associations between MMP-1, $-2,-3,-9$, and -10 and the outcomes were adjusted for the MMP inhibitor TIMP1 , as an indication of MMP activity.

We additionally tested for interaction by variables that may modify the associations between MMPs and TIMP-1 on the one hand and CFPWV or PP on the other (i.e. sex or, in the case of analyses with office PP, cohort) by adding product terms between these variables and MMPs and TIMP-1 to the fully adjusted models. We stratified our analyses whenever significant interactions ( $p$ for interaction $<0.05$ ) were found. 


\section{Results}

\section{Patient characteristics}

Table 4.1 shows patient characteristics according to tertiles of cfPWV ( $n=614)$. Higher cfPWV was significantly associated with higher age, male gender, higher BMI, longer duration of diabetes, higher systolic blood pressure, higher mean arterial pressure, higher PP and lower eGFR. In addition, higher cFPWV was positively associated with a higher prevalence of CVD, a higher degree of albuminuria and a higher degree of retinopathy. Circulating levels of MMP-1, MMP-2, MMP-3, MMP-9, MMP-10 and TIMP-1 were also significantly higher with higher cfPWV.

Table 4.1 Patient characteristics according to tertiles of cfPWV $(n=614)$

\begin{tabular}{|c|c|c|c|c|}
\hline & $\begin{array}{l}\text { First tertile } \\
4.5-8.5 \mathrm{~m} / \mathrm{s} \\
(n=204)\end{array}$ & $\begin{array}{c}\text { Second tertile } \\
8.6-11.3 \mathrm{~m} / \mathrm{s} \\
(n=205)\end{array}$ & $\begin{array}{c}\text { Third tertile } \\
\text { 11.4-23.4 mmHg } \\
(n=205)\end{array}$ & $\begin{array}{l}p \text {-value for } \\
\text { linearity }\end{array}$ \\
\hline Age (years) & $45.2(12.3)$ & $54.9(9.4)$ & $63.0(8.6)$ & $<0.001$ \\
\hline Sex (male/female, \%) & $51 / 49$ & $53 / 47$ & $62 / 38$ & 0.047 \\
\hline BMI $\left(\mathrm{kg} / \mathrm{m}^{2}\right)$ & $24.5(3.5)$ & $25.0(4.2)$ & $25.9(4.0)$ & 0.001 \\
\hline $\mathrm{HbA} 1 \mathrm{c}(\%)$ & $7.9(1.2)$ & $8.2(1.2)$ & $8.0(1.0)$ & 0.054 \\
\hline $\mathrm{HbA} 1 \mathrm{c}(\mathrm{mmol} / \mathrm{mol})$ & $63(13.2)$ & $66(13.3)$ & $64(10.5)$ & 0.054 \\
\hline Duration of diabetes (years) & $20.9(14.4)$ & $33.8(12.6)$ & $42.5(12.5)$ & $<0.001$ \\
\hline Total cholesterol (mmol/l) & $4.69(0.78)$ & $4.64(0.90)$ & $4.75(0.88)$ & 0.454 \\
\hline Smoking at baseline (\%) & 20.1 & 24.4 & 15.1 & 0.183 \\
\hline Systolic blood pressure (mmHg) & $123(12)$ & $131(16)$ & $143(18)$ & $<0.001$ \\
\hline Diastolic blood pressure (mmHg) & $74(9)$ & $76(10)$ & $74(9)$ & 0.145 \\
\hline Mean arterial pressure (mmHg) & $90(9)$ & $94(11)$ & $97(11)$ & $<0.001$ \\
\hline Pulse pressure (mmHg) & $49(10)$ & $55(12)$ & $69(15)$ & $<0.001$ \\
\hline $\operatorname{cfPWV}(\mathrm{m} / \mathrm{s})$ & $7.4[6.5-7.8]$ & $9.8[9.1-10.5]$ & $13.6[12.3-15.5]$ & - \\
\hline Antihypertensive medication (\%) & 43.1 & 77.6 & 89.8 & $<0.001$ \\
\hline eGFR $\left(\mathrm{ml} / \mathrm{min} / 1.73 \mathrm{~m}^{2}\right)$ & $100[85-108]$ & 89 [63-99] & 74 [53-91] & $<0.001$ \\
\hline Cardiovascular disease (\%) & 7.4 & 20.0 & 31.2 & $<0.001$ \\
\hline $\begin{array}{l}\text { Albuminuria (normo-/micro-/ } \\
\text { macro-, \%) }\end{array}$ & $69 / 18 / 13$ & $45 / 26 / 29$ & $32 / 25 / 43$ & $<0.001$ \\
\hline $\begin{array}{l}\text { Retinopathy (no/non-proliferative/ } \\
\text { proliferative/blind, \%) }\end{array}$ & $44 / 41 / 15 / 0$ & $14 / 52 / 32 / 2$ & $5 / 34 / 56 / 5$ & $<0.001$ \\
\hline Z-score InMMP-1 & $-0.23(0.95)$ & $0.11(1.03)$ & $0.08(0.90)$ & $<0.001$ \\
\hline Z-score InMMP-2 & $-0.47(0.95)$ & $-0.02(0.89)$ & $0.43(0.95)$ & $<0.001$ \\
\hline Z-score InMMP-3 & $-0.37(0.91)$ & $0.00(1.00)$ & $0.38(0.84)$ & $<0.001$ \\
\hline Z-score InMMP-9 & $-0.18(0.92)$ & $0.11(0.95)$ & $0.07(0.98)$ & 0.004 \\
\hline Z-score InMMP-10 & $-0.27(0.98)$ & $0.07(1.06)$ & $0.18(0.93)$ & $<0.001$ \\
\hline Z-score InTIMP-1 & $-0.26(0.74)$ & $0.00(1.28)$ & $0.20(0.85)$ & $<0.001$ \\
\hline
\end{tabular}

Data are presented as means (standard deviation), median [inter-quartile range], or percentages, as appropriate. BMI, body mass index; HbA1c, glycated hemoglobin; eGFR, estimated glomerular filtration rate by CKD-EPI formula; MMP, matrix metalloproteinase; TIMP-1, tissue inhibitor of metalloproteinase-1; 


\section{Associations between MMPs, TIMP-1 and cfPWV}

Table 4.2 shows the results of the analyses of the associations between MMPs, TIMP-1 and cfPWV in 614 participants from the PROFIL study. Circulating MMP-3 levels showed an independent association with cfPWV $[0.29 \mathrm{~m} / \mathrm{s}(0.02 ; 0.55)]$ after full adjustment for potential confounders and presence of vascular complications. Circulating levels of MMP-1, MMP-2, MMP-9, MMP-10 and TIMP-1 were not independently associated with cfPWV. As both age and duration of diabetes are significantly associated, and can thus lead to overadjustment, we reanalyzed the data without adjustment for diabetes duration. Results showed an independent association between MMP-2 and cfPWV, with an increase in cfPWV of $0.24 \mathrm{~m} / \mathrm{s}(0.02 ; 0.46)$ per 1 SD higher InMMP-2, after adjustment for the other potential confounders and presence of vascular complications. In addition, the association between MMP-3 and cfPWV became stronger in the fully adjusted model [0.38 (0.11;0.46)]. The associations between MMP-1, MMP-9, MMP-10, and TIMP-1 with cfPWV did not materially change after adjustment for age instead of age and diabetes duration.

Table 4.2 Associations between serum levels of MMP-1, $-2,-3,-9$, and -10 and TIMP-1 and cfPWV $(n=614)$

\begin{tabular}{lcccc}
\hline & Model & $\beta$ & $95 \% \mathrm{Cl}$ & $p$-value \\
\hline MMP-1 & 1 & -0.11 & $-0.31 ; 0.08$ & 0.241 \\
& 2 & -0.11 & $-0.30 ; 0.08$ & 0.263 \\
MMP-2 & 3 & -0.17 & $-0.38 ; 0.03$ & 0.098 \\
& 1 & 0.05 & $-0.16 ; 0.26$ & 0.653 \\
MMP-3 & 2 & 0.06 & $-0.16 ; 0.27$ & 0.598 \\
& 3 & 0.07 & $-0.15 ; 0.28$ & 0.533 \\
MMP-9 & 1 & 0.28 & $0.01 ; 0.54$ & 0.041 \\
& 2 & 0.29 & $0.02 ; 0.55$ & 0.037 \\
& 3 & 0.27 & $0.00 ; 0.54$ & 0.047 \\
MMP-10 & 1 & 0.09 & $-0.10 ; 0.28$ & 0.345 \\
& 2 & 0.09 & $-0.11 ; 0.28$ & 0.397 \\
TIMP-1 & 3 & 0.06 & $-0.14 ; 0.26$ & 0.556 \\
& 1 & -0.07 & $-0.26 ; 0.13$ & 0.493 \\
& 2 & -0.04 & $-0.25 ; 0.16$ & 0.673 \\
\hline
\end{tabular}

$\beta$, standardized regression coefficient: indicates increase in cfPWV (in $\mathrm{m} / \mathrm{s}$ ) per 1 SD increase in InMMPs and InTIMP-1. Cl: confidence interval.

Model 1: Adjusted for age, sex, duration of diabetes, HbA1c, eGFR, and MAP

Model 2: Model 1 + total cholesterol, BMI, smoking, use of antihypertensive medication, and presence of vascular complications

Model 3: Model 2 + TIMP-1 


\section{Associations between MMPs, TIMP-1 and PP}

Supplemental Table S4.1 and S4.2 show the patient characteristics of the three studies separately and according to tertiles of PP, respectively. Table 4.3 shows the results of the pooled data analysis of associations between MMPs, TIMP-1 and office brachial PP. After adjustment for potential confounders, 1 SD higher MMP-1 was inversely associated with PP [-0.83 (-1.50;-0.16)], whereas MMP-2 was positively associated with PP [1.33 $(0.55 ; 2.10)$ ] (Table 4.3 , model 2). These associations did not materially change after additional adjustment for TIMP-1 (Table 4.3, model 3) or mutual adjustment for MMP-2 or MMP-1, respectively. MMP-3, MMP-9, MMP-10 and TIMP-1 were not independently associated with PP (Table 4.3). MMP-9 was only associated with PP after additional adjustment for TIMP-1 [-0.71 (-1.40;-0.01)] (Table 4.3, model 3).

Table 4.3 Associations between circulating levels of MMP-1, -2, -3, -9, and -10 and TIMP-1 and PP (pooled data, $n=1517)$

\begin{tabular}{lcccc}
\hline & Model & $\beta$ & $95 \%$ Cl & $p$-value \\
\hline MMP-1 & 1 & -0.81 & $-1.48 ;-0.15$ & 0.017 \\
& 2 & -0.83 & $-1.50 ;-0.16$ & 0.015 \\
MMP-2 & 3 & -1.08 & $-1.81 ;-0.35$ & 0.004 \\
& 1 & 1.31 & $0.55 ; 2.07$ & 0.001 \\
MMP-3 & 2 & 1.33 & $0.55 ; 2.10$ & 0.001 \\
& 3 & 1.32 & $0.55 ; 2.09$ & 0.001 \\
MMP-9 & 1 & 0.42 & $-0.49 ; 1.32$ & 0.366 \\
& 2 & 0.41 & $-0.50 ; 1.31$ & 0.378 \\
MMP-10 & 3 & 0.39 & $-0.52 ; 1.30$ & 0.401 \\
& 1 & -0.55 & $-1.20 ; 0.10$ & 0.099 \\
& 2 & -0.59 & $-1.25 ; 0.08$ & 0.084 \\
TIMP-1 & 3 & -0.71 & $-1.40 ;-0.01$ & 0.047 \\
& 1 & -0.31 & $-1.01 ; 0.39$ & 0.383 \\
\hline
\end{tabular}

$\beta$, standardized regression coefficient: indicates increase in pulse pressure (in $\mathrm{mmHg}$ ) per $1 \mathrm{SD}$ increase in InMMPs and InTIMP-1. Cl: confidence interval.

Model 1: Adjusted for age, sex, duration of diabetes, HbA1c, eGFR, MAP, cohort and double inclusion.

Model 2: Model 1 + total cholesterol, BMI, smoking, use of antihypertensive medication, and presence of vascular complications.

Model 3: Model 2 + TIMP-1

As brachial BP measurement may overestimated central SBP and PP more in younger individuals, we re-analyzed the data in individuals above 40 years of age $(n=974)$. After full adjustment for potential confounders, 1 SD higher MMP-1 was inversely associated with PP [-1.53 (-2.45;-0.61)], whereas MMP-2 and MMP-3 were positively associated with PP $[1.40(0.45 ; 2.35)]$ and $[1.59(0.48 ; 2.70)]$, respectively. In addition, these three 
markers of ECM remodeling all remained independently associated with office PP when included in the analysis with all MMPs and TIMP-1 together.

In the PROFIL study only, in which data on 24-h brachial and 24-h central PP were available, only MMP-2 was significantly associated with 24-h brachial PP [1.40 (0.47;2.33)] and 24-h central PP [1.43 (0.63;2.23)] (Supplemental Table S4.3). MMP-2 remains significantly associated with 24-h PP measurement when only individuals above 40 were analyzed (data not shown).

\section{Additional analyses}

Adjustment for CVD, albuminuria and retinopathy as separate variables, instead of combined into presence of any vascular complication, did not materially change the results (data not shown). Adjustment for ACE-inhibitors/angiotensin II receptor antagonists instead of antihypertensive therapy did also not materially change the results (data not shown). Additional adjustment, in individuals from the PROFIL study, for use of statins and heart rate did not materially change the associations either between MMPs and cfPWV/office PP or between MMP-2 and 24-h blood pressure measurements (data not shown).

The association between MMP-1 and PP in LEACE differed significantly from those in the other studies ( $p$ for interaction 0.015). MMP-1 was inversely associated with PP in EURODIAB [-1.41 (-2.59;-0.23)] and non-significantly inversely in PROFIL [-0.73 $(-1.64 ; 0.19)]$, whereas a non-significant positive association was observed in LEACE [0.20 $(-1.41 ; 1.81)]$ (Supplemental Table S4.4). No other significant interaction between MMPs/TIMP-1 and cohort was present with regard to the associations between MMPs, TIMP-1 and PP.

No significant sex-associated differences were observed in the associations between MMPs, TIMP-1 and cfPWV or PP ( $p$ for interaction, all >0.05).

\section{Discussion}

In the present study in individuals with type 1 diabetes, we showed that serum levels of MMP-3 were positively associated with cfPWV. In addition, circulating levels of MMP-1 were inversely, and of MMP-2 positively, associated with PP. These associations persisted after adjustment for potential confounders. Circulating levels of MMP-9, MMP-10, and TIMP-1 did not show associations with markers of arterial stiffening.

Our study is the first to show an independent association between serum MMP-3 and cfPWV in individuals with type 1 diabetes, after (extensive) adjustment for potential confounders. To the best of our knowledge, only two studies, in patients without type 1 diabetes, have investigated circulating MMP-3 levels and markers of arterial 
stiffness. $^{31,32}$ Although none of these studies reported independent associations between circulating MMP-3 and cfPWV, one-year treatment with perindopril, compared to placebo, was associated with a significant reduction in cfPWV as well as in plasma MMP-2 and plasma MMP-3 in 17 patients with Marfan Syndrome. ${ }^{31}$ In addition, Sasamura et al. $^{32}$ observed a non-significant positive association between circulating MMP-3 and brachial ankle PWV (baPWV) in a small study of non-diabetic hypertensive patients.

MMP-3 may contribute to arterial stiffness by cleaving matrix collagens (type II, IV, IX, X), elastin, laminin and nidogen, leading to increased collagen turnover and decreased elastin content. MMP-3, similar to MMP-2, can also induce fibroblast-mediated matrix production by cleaving extracellular decorin and releasing TGF- $\beta$ from the extracellular matrix. $^{33}$ In contrast to cfPWV, serum levels of MMP-3 were not associated with office PP nor with 24-h PP measurements. This could be caused by the fact that MMP-3 mediated ECM remodeling might mainly be performed in descending part of the aorta and that its intrinsic actions in the ascending aorta and aortic arch might be limited due the difference in aortic tissue composition. ${ }^{34}$ This could explain the different findings in the associations between MMP-3 on the one hand and CFPWV and PP measurements on the other. However, as office PP may overestimate central PP more in younger (healthy) individuals, as indicated by Laurent et al., ${ }^{2}$ we showed that MMP-3 was also associated with office PP in individuals with type 1 diabetes above the age of 40 and this is in line with finding of MMP-3 and cfPWV.

Serum MMP-2 levels and cfPWV were not independently associated in our study, whereas circulating MMP-2 was associated with both office PP as well as 24-h PP measurements. Our result differs from studies performed in non-diabetic individuals, in whom positive associations between circulating levels of MMP-2 and cfPWV were observed. ${ }^{12-14}$ However, our study is in accordance with the study by Coutinho et al., ${ }^{17}$ in which a positive association between plasma MMP-2 and PP was observed in normotensive and hypertensive African-Americans in the GENOA study. These results were also supported by studies at tissue level. ${ }^{8,35,36}$ Arteries of dialyzed chronic kidney disease patients compared to those of non-dialyzed chronic kidney disease patients and kidney donors ${ }^{35}$ showed higher MMP-2 activity, which was associated with lower elastic fiber content and greater arterial stiffness. In addition, renal transplant recipients with diabetes had stiffer arteries compared to recipients without diabetes and controls, and this was significantly associated with higher MMP-2 and MMP-9 activity in the arterial wall. $^{8}$ Next, in aortic tissue of aging rats with higher systolic blood pressure, higher intimal and medial MMP-2 levels and MMP-2/TIMP-2 ratios were observed. ${ }^{36}$ These increases were accompanied by greater vascular intima and media thickness as well as higher medial type III collagen and lower elastin content. ${ }^{36} \mathrm{MMP}-2$ has the ability to release transforming growth factor $\beta$ (TGF- $\beta$ ) from the ECM. ${ }^{33}$ A TGF- $\beta$-induced increase in fibroblast-mediated ECM production thus may be a plausible mechanism to explain the strong association between MMP-2 and arterial stiffening. ${ }^{37}$ 
Duration of diabetes appeared to be the major confounder in the association between MMP-2 and cfPWV and was significantly correlated with age. If duration of diabetes would not be taken into account, a positive association was in fact observed, which is in line with the association between MMP-2 and office PP as well as 24-h PP measurements. Therefore, the model with adjustment for both age and diabetes duration may have been overadjusted and our results are consistent with an important role for MMP-2 in pathophysiological vascular remodeling. However, we cannot exclude that both age and duration of diabetes were the major confounders in the association between MMP-2 and cfPWV.

We also showed that serum MMP-1 was borderline significantly inversely associated with cfPWV after adjustment for potential confounders and TIMP-1 levels and this is in contrast to two studies in non-diabetic hypertensive patients in whom (pro)MMP-1 was significantly and positively associated with cfPWV. ${ }^{9,10}$ Patients with diabetes were excluded in both studies and this may explain the different results. However, the inverse association between MMP-1 and CFPWV is in fact consistent with the inverse association between MMP-1 and PP that we observed. The difference in the associations between MMP-1 on the one hand and CfPWV and PP on the other might be caused by the differences in vascular wall content in the various parts of the aorta. ${ }^{34}$ Circulating levels of MMP-1 were inversely associated with PP. To our knowledge, correlations between circulating (pro)MMP-1 and PP have only been published in studies that did not include individuals with type 1 diabetes, ${ }^{10,38}$ and were not adjusted for potential confounders. In our crude analysis, 1SD higher InMMP-1 was associated with a higher PP [1.36 $(0.49 ; 2.23)]$. However the association inverted after adjustment for potential confounders, with MAP being the most influential confounder. The fact that higher tissue MMP-1 levels have been associated with thoracic aortic aneurysm and thoracic aortic dissection $^{39}$ also indicates that higher MMP-1 levels may result in decreased thoracic aortic collagen content and/or concentration leading to decreased arterial stiffness. Nonetheless, evidence so far on the role of MMP-1 in collagen degradation and/or turnover and arterial stiffness is scarce and should be further investigated.

Published data on MMP-9 and PWV are contradictory. The lack of association between MMP-9 and cfPWV in the present study was in agreement with studies in patients with essential hypertension, ${ }^{15}$ coronary heart disease ${ }^{16}$ and bicuspid aortic valves. ${ }^{13}$ In contrast, Yasmin et al. ${ }^{12}$ observed a positive and independent association between MMP-9 and cFPWV in both hypertensive patients and healthy individuals. In addition, serum MMP-9, but also MMP-3 and TIMP-1, was correlated with invasive aortic PWV measurements in patients with aortic stenosis. ${ }^{40}$ However, the latter correlations were unadjusted for potential confounders. In contrast to all previous studies, an inverse association was observed serum MMP-9 and cfPWV in healthy subjects. ${ }^{41}$ In accordance with our result, Coutinho et al. did also not observe an association between MMP-9, as well as TIMP-1, and PP. ${ }^{17}$ Therefore, the role of MMP-9 in arterial stiffness remains unclear and warrants further investigation. 
Our finding of a lack of association between TIMP-1 levels and cfPWV is supported by various other studies. ${ }^{13,16,32}$ Only the study by Tan et $a .^{15}$ observed an independent association between TIMP-1 and CfPWV in hypertensive patients, but patients with macrovascular complications, diabetes and possible inflammatory diseases were excluded from this study.

Various studies investigated associations between circulating MMP- $1,{ }^{42} \mathrm{MMP}-2,{ }^{43,44}$ MMP-3, ${ }^{42}$ MMP-9. ${ }^{43-46}$ MMP-10, ${ }^{42}$ and TIMP-1 ${ }^{43}$ with other markers of arterial stiffness in both type $1^{45}$ and type $2^{42-44,46}$ diabetic individuals, but did not find significant associations.

\section{Limitations}

Several limitations are applicable to our study. First, we used PP as marker of arterial stiffness, but this only presents indirect information on arterial stiffness. However, it has been shown to be an independent predictor of cardiovascular events and all-cause mortality in type 1 diabetic patients. ${ }^{1,4,5}$ Second, although our study also included cfPWV measurements, the current gold standard for arterial stiffness, these were only available in one of the three included studies. Thirdly, we used circulating levels of MMPs and TIMP-1, because these can easily be obtained, but we do not know whether or not plasma levels truly reflect tissue levels or if they originate from one or more specific tissues. Finally, we cannot draw conclusions on the pathophysiological mechanism regarding the development of arterial stiffness, because of the cross-sectional design of our studies. In addition, the significant difference, between the LEACE and the other two studies, in the association between MMP-1 and PP may have been caused by the play of chance. Despite these limitations, our results showed largely consistent results throughout the three different studies with regard to the associations between MMP-1, MMP-2 and PP in individuals with type 1 diabetes.

\section{Conclusion}

In individuals with type 1 diabetes, MMP-1 (inversely) and MMP-2 and -3 (both positively) were associated with markers of arterial stiffening (PP and CfPWV). The results suggest that better understanding of altered ECM remodeling in patients with type 1 diabetes could provide a potential therapeutic target, which could lead to decreased arterial stiffness and improved outcomes. 


\section{References}

1. Stehouwer CD, Henry RM, Ferreira I: Arterial stiffness in diabetes and the metabolic syndrome: a pathway to cardiovascular disease. Diabetologia 2008;51:527-539

2. Laurent S, Cockcroft J, Van Bortel L, Boutouyrie P, Giannattasio C, Hayoz D, Pannier B, Vlachopoulos C, Wilkinson I, Struijker-Boudier H: Expert consensus document on arterial stiffness: methodological issues and clinical applications. Eur Heart J 2006;27:2588-2605

3. Theilade S, Lajer M, Persson F, Joergensen C, Rossing P: Arterial stiffness is associated with cardiovascular, renal, retinal, and autonomic disease in type 1 diabetes. Diabetes Care 2013;36:715-721

4. Theilade S, Lajer M, Jorsal A, Tarnow L, Parving HH, Rossing P: Arterial stiffness and endothelial dysfunction independently and synergistically predict cardiovascular and renal outcome in patients with type 1 diabetes. Diabet Med 2012;29:990-994

5. Gordin D, Waden J, Forsblom C, Thorn L, Rosengard-Barlund M, Tolonen N, Saraheimo M, Harjutsalo V, Groop PH: Pulse pressure predicts incident cardiovascular disease but not diabetic nephropathy in patients with type 1 diabetes (The FinnDiane Study). Diabetes Care 2011;34:886-891

6. Galis ZS, Khatri JJ: Matrix metalloproteinases in vascular remodeling and atherogenesis: the good, the bad, and the ugly. Circ Res 2002;90:251-262

7. Fontana V, Silva PS, Gerlach RF, Tanus-Santos JE: Circulating matrix metalloproteinases and their inhibitors in hypertension. Clin Chim Acta 2012;413:656-662

8. Chung AW, Yang HH, Sigrist MK, Brin G, Chum E, Gourlay WA, Levin A: Matrix metalloproteinase-2 and -9 exacerbate arterial stiffening and angiogenesis in diabetes and chronic kidney disease. Cardiovasc Res 2009;84:494-504

9. McNulty M, Mahmud A, Spiers P, Feely J: Collagen type-I degradation is related to arterial stiffness in hypertensive and normotensive subjects. J Hum Hypertens 2006;20:867-873

10. Stakos DA, Tziakas DN, Chalikias GK, Mitrousi K, Tsigalou C, Boudoulas H: Associations between collagen synthesis and degradation and aortic function in arterial hypertension. Am J Hypertens 2010;23:488-494

11. Donley DA, Fournier SB, Reger BL, DeVallance E, Bonner DE, Olfert IM, Frisbee JC, Chantler PD: Aerobic exercise training reduces arterial stiffness in metabolic syndrome. J Appl Physiol (1985) 2014;116: 1396-1404

12. Yasmin, McEniery CM, Wallace S, Dakham Z, Pulsalkar P, Maki-Petaja K, Ashby MJ, Cockcroft JR, Wilkinson IB: Matrix metalloproteinase-9 (MMP-9), MMP-2, and serum elastase activity are associated with systolic hypertension and arterial stiffness. Arterioscler Thromb Vasc Biol 2005;25:372

13. Tzemos N, Lyseggen E, Silversides C, Jamorski M, Tong JH, Harvey P, Floras J, Siu S: Endothelial function, carotid-femoral stiffness, and plasma matrix metalloproteinase-2 in men with bicuspid aortic valve and dilated aorta. J Am Coll Cardiol 2010;55:660-668

14. Smith ER, Tomlinson LA, Ford ML, McMahon LP, Rajkumar C, Holt SG: Elastin degradation is associated with progressive aortic stiffening and all-cause mortality in predialysis chronic kidney disease. Hypertension 2012;59:973-978

15. Tan J, Hua Q, Xing X, Wen J, Liu R, Yang Z: Impact of the metalloproteinase-9/tissue inhibitor of metalloproteinase-1 system on large arterial stiffness in patients with essential hypertension. Hypertens Res 2007;30:959-963

16. Zureik M, Beaudeux JL, Courbon D, Benetos A, Ducimetiere P: Serum tissue inhibitors of metalloproteinases 1 (TIMP-1) and carotid atherosclerosis and aortic arterial stiffness. J Hypertens 2005;23:2263-2268

17. Coutinho T, Turner ST, Mosley TH, Kullo IJ: Biomarkers associated with pulse pressure in AfricanAmericans and non-Hispanic whites. Am J Hypertens 2012;25:145-151

18. Chaturvedi N, Sjoelie AK, Porta M, Aldington SJ, Fuller JH, Songini M, Kohner EM: Markers of insulin resistance are strong risk factors for retinopathy incidence in type 1 diabetes. Diabetes Care 2001;24:284-289

19. Chaturvedi N, Bandinelli S, Mangili R, Penno G, Rottiers RE, Fuller JH: Microalbuminuria in type 1 diabetes: rates, risk factors and glycemic threshold. Kidney Int 2001;60:219-227 
20. Schram MT, Chaturvedi N, Schalkwijk C, Giorgino F, Ebeling P, Fuller JH, Stehouwer CD: Vascular risk factors and markers of endothelial function as determinants of inflammatory markers in type 1 diabetes: the EURODIAB Prospective Complications Study. Diabetes Care 2003;26:2165-2173

21. Astrup AS, Tarnow L, Rossing P, Pietraszek L, Riis Hansen P, Parving HH: Improved prognosis in type 1 diabetic patients with nephropathy: a prospective follow-up study. Kidney Int 2005;68:1250-1257

22. Schram MT, Chaturvedi N, Fuller JH, Stehouwer CD, Group EPCS: Pulse pressure is associated with age and cardiovascular disease in type 1 diabetes: the Eurodiab Prospective Complications Study. J Hypertens 2003;21:2035-2044

23. Tarnow L, Cambien F, Rossing P, Nielsen FS, Hansen BV, Lecerf L, Poirier O, Danilov S, Parving HH: Lack of relationship between an insertion/deletion polymorphism in the angiotensin I-converting enzyme gene and diabetic nephropathy and proliferative retinopathy in IDDM patients. Diabetes 1995;44:489-494

24. Theilade S, Lyngbaek S, Hansen TW, Eugen-Olsen J, Fenger M, Rossing P, Jeppesen JL: Soluble urokinase plasminogen activator receptor levels are elevated and associated with complications in patients with type 1 diabetes. Journal of internal medicine 2015;277:362-371

25. Nair D, Tan SY, Gan HW, Lim SF, Tan J, Zhu M, Gao H, Chua NH, Peh WL, Mak KH: The use of ambulatory tonometric radial arterial wave capture to measure ambulatory blood pressure: the validation of a novel wrist-bound device in adults. J Hum Hypertens 2008;22:220-222

26. Theilade S, Joergensen C, Persson F, Lajer M, Rossing P: Ambulatory tonometric blood pressure measurements in patients with diabetes. Diabetes Technol Ther 2012;14:453-456

27. O'Brien E, Asmar R, Beilin L, Imai Y, Mallion JM, Mancia G, Mengden T, Myers M, Padfield P, Palatini P, Parati G, Pickering T, Redon J, Staessen J, Stergiou G, Verdecchia P: European Society of Hypertension recommendations for conventional, ambulatory and home blood pressure measurement. J Hypertens 2003;21:821-848

28. Levey AS, Stevens LA, Schmid CH, Zhang YL, Castro AF, 3rd, Feldman HI, Kusek JW, Eggers P, Van Lente F, Greene T, Coresh J: A new equation to estimate glomerular filtration rate. Ann Intern Med 2009;150:604-612

29. Koivisto VA, Stevens LK, Mattock M, Ebeling P, Muggeo M, Stephenson J, Idzior-Walus B: Cardiovascular disease and its risk factors in IDDM in Europe. EURODIAB IDDM Complications Study Group. Diabetes Care 1996;19:689-697

30. Aldington SJ, Kohner EM, Meuer S, Klein R, Sjolie AK: Methodology for retinal photography and assessment of diabetic retinopathy: the EURODIAB IDDM complications study. Diabetologia 1995;38:437-444

31. Ahimastos AA, Aggarwal A, D'Orsa KM, Formosa MF, White AJ, Savarirayan R, Dart AM, Kingwell BA: Effect of perindopril on large artery stiffness and aortic root diameter in patients with Marfan syndrome: a randomized controlled trial. Jama 2007;298:1539-1547

32. Sasamura H, Kitamura Y, Nakamura M, Ryuzaki M, Saruta T: Effects of the angiotensin receptor blocker candesartan on arterial stiffness and markers of extracellular matrix metabolism in patients with essential hypertension. Clin Exp Hypertens 2006;28:511-520

33. Imai K, Hiramatsu A, Fukushima D, Pierschbacher MD, Okada Y: Degradation of decorin by matrix metalloproteinases: identification of the cleavage sites, kinetic analyses and transforming growth factorbeta1 release. Biochem J 1997;322 ( Pt 3):809-814

34. Tsamis A, Krawiec JT, Vorp DA: Elastin and collagen fibre microstructure of the human aorta in ageing and disease: a review. J R Soc Interface 2013;10:20121004

35. Chung AW, Yang HH, Kim JM, Sigrist MK, Chum E, Gourlay WA, Levin A: Upregulation of matrix metalloproteinase-2 in the arterial vasculature contributes to stiffening and vasomotor dysfunction in patients with chronic kidney disease. Circulation 2009;120:792-801

36. Wang M, Lakatta EG: Altered regulation of matrix metalloproteinase-2 in aortic remodeling during aging. Hypertension 2002;39:865-873

37. Ruiz-Ortega M, Rodriguez-Vita J, Sanchez-Lopez E, Carvajal G, Egido J: TGF-beta signaling in vascular fibrosis. Cardiovasc Res 2007;74:196-206

38. Ishikawa J, Kario K, Matsui Y, Shibasaki S, Morinari M, Kaneda R, Hoshide S, Eguchi K, Hojo Y, Shimada K: Collagen metabolism in extracellular matrix may be involved in arterial stiffness in older hypertensive patients with left ventricular hypertrophy. Hypertens Res 2005;28:995-1001 
39. Koullias GJ, Ravichandran P, Korkolis DP, Rimm DL, Elefteriades JA: Increased tissue microarray matrix metalloproteinase expression favors proteolysis in thoracic aortic aneurysms and dissections. Ann Thorac Surg 2004;78:2106-2110; discussion 2110-2101

40. Liu PY, Tsai WC, Lin CC, Hsu CH, Haung YY, Chen JH: Invasive measurements of pulse wave velocity correlate with the degree of aortic valve calcification and severity associated with matrix metalloproteinases in elderly patients with aortic valve stenosis. Clin Sci (Lond) 2004;107:415-422

41. Vlachopoulos C, Aznaouridis K, Dima I, loakeimidis N, Vasiliadou C, Zervoudaki A, Gialernios T, Stefanadis C: Negative association between serum levels of matrix metalloproteinases- 2 and -9 and aortic stiffness in healthy adults. Int J Cardiol 2007;122:232-238

42. Goncalves I, Bengtsson E, Colhoun HM, Shore AC, Palombo C, Natali A, Edsfeldt A, Duner P, Fredrikson GN, Bjorkbacka H, Ostling G, Aizawa K, Casanova F, Persson M, Gooding K, Strain D, Khan F, Looker HC, Adams F, Belch J, Pinnoli S, Venturi E, Kozakova M, Gan LM, Schnecke V, Nilsson J, Consortium S: Elevated Plasma Levels of MMP-12 Are Associated With Atherosclerotic Burden and Symptomatic Cardiovascular Disease in Subjects With Type 2 Diabetes. Arterioscler Thromb Vasc Biol 2015;35: 1723-1731

43. Papazafiropoulou A, Perrea D, Moyssakis I, Kokkinos A, Katsilambros N, Tentolouris N: Plasma levels of MMP-2, MMP-9 and TIMP-1 are not associated with arterial stiffness in subjects with type 2 diabetes mellitus. J Diabetes Complications 2010;24:20-27

44. Uzui H, Nakano A, Mitsuke Y, Geshi T, Sakata J, Sarazawa K, Morishita T, Satou T, Ishida K, Lee JD: Acarbose treatments improve arterial stiffness in patients with type 2 diabetes mellitus. J Diabetes Investig 2011;2:148-153

45. Rogowicz-Frontczak A, Pilacinski S, Araszkiewicz A, Zozulinska-Ziolkiewicz D, Wykretowicz A, WieruszWysocka B: C-Reactive protein and soluble intracellular adhesion molecule-1 are related to pulse wave reflection in type 1 diabetes 1C-1. J Diabetes 2014;6:577-585

46. Kiyici S, Ersoy C, Kaderli A, Fazlioglu M, Budak F, Duran C, Gul OO, Sigirli D, Baran I, Tuncel E, Erturk E, Imamoglu S: Effect of rosiglitazone, metformin and medical nutrition treatment on arterial stiffness, serum MMP-9 and MCP-1 levels in drug naive type 2 diabetic patients. Diabetes Res Clin Pract 2009;86:44-50 


\section{Supplemental data}

Table S4.1 Baseline characteristics of the three studies

\begin{tabular}{|c|c|c|c|}
\hline & $\begin{array}{c}\text { EURODIAB } \\
(n=509)\end{array}$ & $\begin{array}{l}\text { LEACE } \\
(n=370)\end{array}$ & $\begin{array}{l}\text { PROFIL } \\
(n=638)\end{array}$ \\
\hline Age (years) & $39.6(10.0)$ & $41.8(9.6)$ & $54.6(12.6)$ \\
\hline Sex (male/female, \%) & $52 / 48$ & $61 / 39$ & $55 / 45$ \\
\hline $\mathrm{BMI}\left(\mathrm{kg} / \mathrm{m}^{2}\right)$ & $24.5(3.2)$ & $23.9(2.9)$ & $25.2(4.0)$ \\
\hline $\mathrm{HbA1c}(\%)$ & $8.5(1.6)$ & $9.1(1.4)$ & $8.0(1.4)$ \\
\hline $\mathrm{HbA1c}(\mathrm{mmol} / \mathrm{mol})$ & $70(17.6)$ & $76(15.8)$ & $64(12.5)$ \\
\hline Duration of diabetes (years) & $21.5(9.5)$ & $27.8(8.0)$ & $32.6(16.0)$ \\
\hline Total cholesterol (mmol/l) & $5.31(1.15)$ & $5.23(1.19)$ & $4.69(0.86)$ \\
\hline Smoking at start of study (\%) & 31.0 & 46.5 & 20.8 \\
\hline Systolic blood pressure (mmHg) & $123(20)$ & $142(23)$ & $132(18)$ \\
\hline Diastolic blood pressure $(\mathrm{mmHg})$ & $75(11)$ & $81(13)$ & $74(9)$ \\
\hline Mean arterial pressure $(\mathrm{mmHg})$ & $91(12)$ & $102(14)$ & $94(11)$ \\
\hline Pulse pressure (mmHg) & $48(17)$ & $61(18)$ & $58(15)$ \\
\hline Pulse wave velocity $(\mathrm{m} / \mathrm{s})$ & - & - & $9.8[7.8-12.3]$ \\
\hline Antihypertensive medication (\%) & 31.0 & 43.2 & 71.0 \\
\hline Statins $(\%)$ & - & - & 59.7 \\
\hline eGFR $\left(\mathrm{ml} / \mathrm{min} / 1.73 \mathrm{~m}^{2}\right)$ & $102[87-112]$ & 101 [75-113] & $88[65-100]$ \\
\hline Cardiovascular disease (\%) & 24.2 & 6.5 & 20.7 \\
\hline Albuminuria (normo-/micro-/macro-, \%) & $61 / 16 / 23$ & $46 / 0 / 54$ & $47 / 24 / 29$ \\
\hline $\begin{array}{l}\text { Retinopathy (no/non-proliferative/ } \\
\text { proliferative/blind, \%) }\end{array}$ & $45 / 26 / 29 / 0$ & $16 / 42 / 42 / 0$ & $21 / 42 / 34 / 3$ \\
\hline $\mathrm{MMP}-1(\mathrm{ng} / \mathrm{ml})$ & $11.8[6.7-19.0]$ & $3.2[1.9-5.7]$ & $20.9[13.0-30.6]$ \\
\hline MMP-2 (ng/ml) & $106[98-115]$ & $200[173-236]$ & 142 [128-159] \\
\hline MMP-3 (ng/ml) & $15.8[9.9-24.6]$ & $15.1[9.5-24.4]$ & $19.2[12.8-28.8]$ \\
\hline MMP-9 (ng/ml) & 118 [76-187] & $25.0[15.7-44.6]$ & $161[108-241]$ \\
\hline MMP-10 (pg/ml) & $1207[882-1881]$ & 723 [499-980] & $1167[868-1671]$ \\
\hline TIMP-1 (ng/ml) & $290[238-338]$ & 179 [146-233] & 303 [262-353] \\
\hline
\end{tabular}

Data are presented as means (standard deviation), median [inter-quartile range], or percentages, as appropriate. BMI, body mass index; HbA1c, glycated hemoglobin; eGFR, estimated glomerular filtration rate by CKD-EPI formula; MMP, matrix metalloproteinase; TIMP-1, tissue inhibitor of metalloproteinase-1; blind*, blindness was present in patients in the PROFIL study 
Table S4.2 Patient characteristics according to tertiles of PP $(n=1517)$

\begin{tabular}{|c|c|c|c|c|}
\hline & $\begin{array}{c}\text { First tertile } \\
17-46 \mathrm{mmHg} \\
(\mathrm{n}=517)\end{array}$ & $\begin{array}{c}\text { Second tertile } \\
\text { 47-59 } \mathrm{mmHg} \\
(\mathrm{n}=488)\end{array}$ & $\begin{array}{c}\text { Third tertile } \\
60-129 \mathrm{mmHg} \\
(\mathrm{n}=512)\end{array}$ & $\begin{array}{l}p \text {-value for } \\
\text { linearity }\end{array}$ \\
\hline Age (years) & $39.8(10.8)$ & $46.3(12.1)$ & $53.3(12.6)$ & $<0.001$ \\
\hline Sex (male/female, \%) & $52 / 48$ & $57 / 43$ & $57 / 43$ & 0.229 \\
\hline BMI $\left(\mathrm{kg} / \mathrm{m}^{2}\right)$ & $24.2(3.1)$ & $24.7(3.6)$ & $25.0(3.8)$ & $<0.001$ \\
\hline $\mathrm{HbA} 1 \mathrm{c}(\%)$ & $8.4(1.5)$ & $8.3(1.4)$ & $8.6(1.5)$ & 0.046 \\
\hline $\mathrm{HbA} 1 \mathrm{c}(\mathrm{mmol} / \mathrm{mol})$ & $69(16.4)$ & $68(14.8)$ & $71(16.0)$ & 0.046 \\
\hline Duration of diabetes (years) & $21.2(10.7)$ & $27.3(12.3)$ & $34.8(13.0)$ & $<0.001$ \\
\hline Total cholesterol (mmol/l) & $4.91(0.99)$ & $4.99(1.06)$ & $5.19(1.19)$ & $<0.001$ \\
\hline Smoking at baseline (\%) & 30.6 & 33.0 & 28.1 & 0.248 \\
\hline Systolic blood pressure (mmHg) & $114(12)$ & $128(12)$ & $152(17)$ & $<0.001$ \\
\hline Diastolic blood pressure (mmHg) & $76(10)$ & $76(12)$ & $77(12)$ & 0.010 \\
\hline Mean arterial pressure $(\mathrm{mmHg})$ & $88(10)$ & $93(12)$ & $102(12)$ & $<0.001$ \\
\hline Pulse pressure (mmHg) & $39(6)$ & $52(4)$ & $75(13)$ & - \\
\hline Antihypertensive medication (\%) & 29.8 & 50.8 & 71.1 & $<0.001$ \\
\hline eGFR $\left(\mathrm{ml} / \mathrm{min} / 1.73 \mathrm{~m}^{2}\right)$ & $104[90-114]$ & $98[79-108]$ & 83 [59-99] & $<0.001$ \\
\hline Cardiovascular disease (\%) & 14.9 & 16.2 & 24.0 & $<0.001$ \\
\hline $\begin{array}{l}\text { Albuminuria (normo-/micro-/macro-, } \\
\%)\end{array}$ & $68 / 13 / 19$ & $51 / 16 / 33$ & $37 / 17 / 46$ & $<0.001$ \\
\hline $\begin{array}{l}\text { Retinopathy (no/non-proliferative/ } \\
\text { proliferative/blind*,\%) }\end{array}$ & $44 / 33 / 22 / 1$ & $26 / 38 / 35 / 1$ & $13 / 38 / 47 / 2$ & $<0.001$ \\
\hline Z-score InMMP-1 & $-0.06(0.97)$ & $0.05(1.04)$ & $0.01(0.99)$ & 0.288 \\
\hline Z-score InMMP-2 & $-0.47(0.80)$ & $0.00(0.89)$ & $0.48(1.05)$ & $<0.001$ \\
\hline Z-score InMMP-3 & $-0.19(0.96)$ & $-0.07(1.02)$ & $0.26(0.97)$ & $<0.001$ \\
\hline Z-score InMMP-9 & $0.07(0.95)$ & $-0.01(1.01)$ & $-0.06(1.04)$ & 0.045 \\
\hline Z-score InMMP-10 & $-0.01(0.99)$ & $0.02(1.01)$ & $-0.01(1.00)$ & 0.985 \\
\hline Z-score InTIMP-1 & $-0.08(1.00)$ & $-0.04(1.09)$ & $0.12(0.90)$ & 0.002 \\
\hline
\end{tabular}

Data are presented as means (standard deviation), median [inter-quartile range], or percentages, as appropriate. BMI, body mass index; HbA1c, glycated hemoglobin; eGFR, estimated glomerular filtration rate by CKD-EPI formula; MMP, matrix metalloproteinase; TIMP-1, tissue inhibitor of metalloproteinase-1; *a category for blindness was only included in the PROFIL study 
Table S4.3 Associations between circulating levels of MMP-1, -2, -3, -9, and -10 and TIMP-1 and 24-h PP measurements in PROFIL

\begin{tabular}{lccccccc}
\hline & & \multicolumn{3}{c}{$\begin{array}{c}\text { PROFIL } \\
\text { brachial } 24 \mathrm{~h} \\
(\mathrm{n}=638)\end{array}$} & & & $\begin{array}{c}\text { PROFIL } \\
\text { Central 24h } \\
(\mathrm{n}=638)\end{array}$ \\
\hline MMP-1 & Model & $\beta$ & $95 \% \mathrm{Cl}$ & $\mathrm{p}$-value & $\beta$ & $95 \% \mathrm{Cl}$ & $\mathrm{p}$-value \\
& 1 & 0.01 & $-0.81 ; 0.83$ & 0.986 & -0.02 & $-0.73 ; 0.69$ & 0.953 \\
& 2 & 0.04 & $-0.78 ; 0.86$ & 0.924 & 0.02 & $-0.69 ; 0.73$ & 0.960 \\
MMP-2 & 3 & -0.08 & $-0.96 ; 0.80$ & 0.854 & -0.04 & $-0.80 ; 0.72$ & 0.912 \\
& 1 & 1.48 & $0.56 ; 2.40$ & 0.002 & 1.51 & $0.71 ; 2.31$ & $<0.001$ \\
MMP-3 & 2 & 1.40 & $0.47 ; 2.33$ & 0.003 & 1.43 & $0.63 ; 2.23$ & $<0.001$ \\
& 3 & 1.43 & $0.50 ; 2.36$ & 0.003 & 1.45 & $0.65 ; 2.25$ & $<0.001$ \\
MMP-9 & 1 & 0.26 & $-0.81 ; 1.34$ & 0.631 & 0.22 & $-0.72 ; 1.15$ & 0.651 \\
& 2 & 0.27 & $-0.81 ; 1.35$ & 0.620 & 0.24 & $-0.70 ; 1.17$ & 0.619 \\
& 3 & 0.24 & $-0.84 ; 1.33$ & 0.659 & 0.22 & $-0.72 ; 1.16$ & 0.641 \\
MMP-10 & 1 & -0.12 & $-0.92 ; 0.68$ & 0.772 & -0.22 & $-0.92 ; 0.47$ & 0.533 \\
& 2 & -0.02 & $-0.84 ; 0.80$ & 0.959 & -0.11 & $-0.83 ; 0.60$ & 0.756 \\
& 3 & -0.09 & $-0.93 ; 0.75$ & 0.829 & -0.15 & $-0.88 ; 0.58$ & 0.681 \\
& 1 & 0.18 & $-0.69 ; 1.04$ & 0.687 & 0.15 & $-0.60 ; 0.90$ & 0.695 \\
& 2 & 0.13 & $-0.77 ; 1.02$ & 0.780 & 0.16 & $-0.61 ; 0.94$ & 0.678 \\
& 3 & 0.09 & $-0.81 ; 0.99$ & 0.839 & 0.15 & $-0.63 ; 0.93$ & 0.710 \\
& 1 & 0.20 & $-0.66 ; 1.06$ & 0.646 & 0.03 & $-0.72 ; 0.77$ & 0.941 \\
& 2 & 0.34 & $-0.53 ; 1.20$ & 0.444 & 0.17 & $-0.58 ; 0.92$ & 0.660 \\
\hline
\end{tabular}

$\beta$, standardized regression coefficient: indicates increase in pulse pressure (in $\mathrm{mmHg}$ ) per $1 \mathrm{SD}$ increase in InMMPs and InTIMP-1. Cl: confidence interval.

Model 1: Adjusted for age, sex, duration of diabetes, HbA1c, eGFR, and MAP

Model 2: Model 1 + total cholesterol, BMI, smoking, use of antihypertensive medication, and presence of vascular complications

Model 3: Model $2+$ TIMP-1 


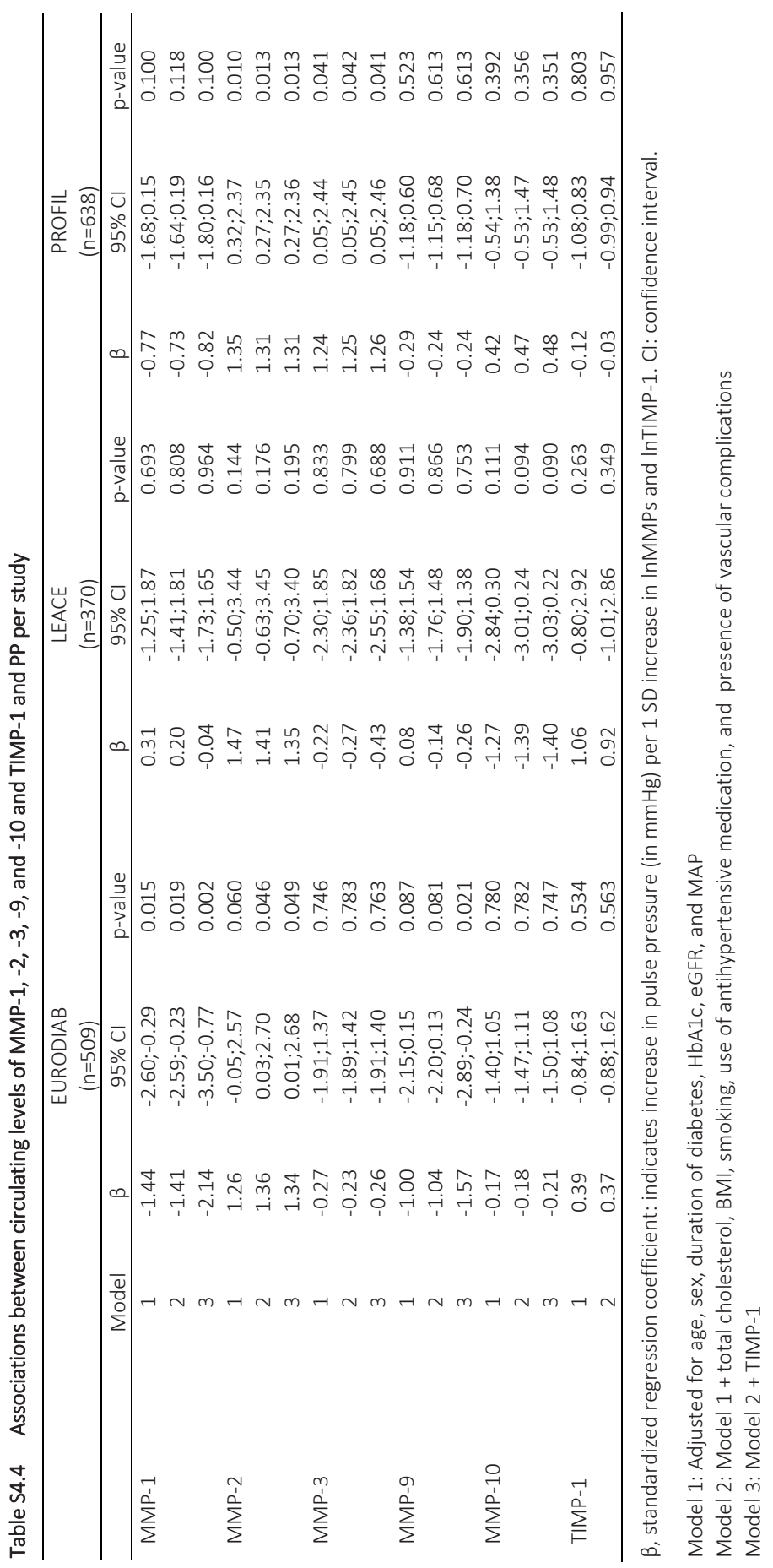




\section{Chapter}

Associations between advanced glycation endproducts and matrix metalloproteinases and its inhibitor in individuals with type 1 diabetes 


\section{Abstract}

\section{Objective}

Advanced glycation endproducts (AGEs) and altered extracellular matrix remodeling by matrix metalloproteinases (MMPs) and tissue inhibitor of metalloproteinase (TIMP) are associated with vascular complications in type 1 diabetes. Experimental studies have shown that AGEs regulate the production of MMPs and/or TIMP-1; however, data in humans on the association between AGEs and MMPS/TIMP-1 are currently lacking. Therefore, we investigated associations between serum levels of specific AGEs and MMP-1, -2, -3, -9, and -10, and TIMP-1 in individuals with type 1 diabetes.

\section{Methods}

We included 670 individuals with type 1 diabetes and determined serum levels of protein-bound AGEs ( $N^{\varepsilon}$-(carboxymethyl)lysine (CML), $N^{\varepsilon}$-(carboxyethyl)lysine (CEL), 5-hydro-5-methylimidazolone (MG-H1) and pentosidine), as well as MMP-1, -2, -3, -9, and -10, and TIMP-1. We performed linear regression analyses to investigate associations between AGEs on the one hand and markers of the MMP-TIMP system on the other. Analyses were adjusted for age, sex, $\mathrm{HbA} 1 \mathrm{C}$ and duration of diabetes, and additionally for other potential confounders and presence of vascular complication.

\section{Results}

After full adjustment, levels of $\mathrm{CML}$ were positively associated with levels of MMP-2 $[\beta=0.11(0.03 ; 0.19)]$ and inversely with MMP-9 $[\beta=-0.13(-0.22 ;-0.03)]$. CEL was positively associated with MMP-3 $0.08(0.02 ; 0.14)]$ and TIMP-1 [0.08 (0.01;0.16)]. MGH1 was only associated with TIMP-1 $[0.09(0.02 ; 0.17)]$, whereas pentosidine was not associated with MMPs or TIMP-1.

\section{Conclusions}

In the present study, we showed independent associations between several AGEs and markers of the MMP-TIMP system, which indicate specific AGE-MMP/TIMP-1 interactions potentially contributing to vascular complications in patients with type 1 diabetes. 


\section{Introduction}

The mechanisms leading to vascular complications in individuals with type 1 diabetes have not been fully elucidated. One pathway through which hyperglycemia may lead to vascular complications is the formation of advanced glycation endproducts (AGEs). AGEs are a heterogeneous group of glucose-modified amino acids including $\mathrm{N}^{\varepsilon}$-(carboxymethyl)lysine (CML), $\quad \mathrm{N}^{\varepsilon}$-(carboxyethyl)lysine (CEL), 5-hydro-5-methylimidazolone (MG-H1) and the cross-link pentosidine. AGEs have been associated with incident CVD and all-cause mortality in individuals with type 1 diabetes. ${ }^{1}$ The mechanisms by which AGEs can contribute to diabetic complications include the modification of intracellular proteins, the formation of extracellular cross-links and binding of extracellular AGEs to their receptors (RAGE). ${ }^{2}$ This causes pathological changes in gene expression as well as induction of the production of pro-inflammatory markers, cytokines, growth factors and matrix metalloproteinases (MMPs). ${ }^{2,3}$ MMP are enzymes involved in extracellular matrix (ECM) remodeling, and we and others have shown associations of matrix metalloproteinase-2 (MMP-2), ${ }^{4-6} \mathrm{MMP}-3,{ }^{6} \mathrm{MMP}-9,{ }^{4} \mathrm{MMP}^{-10^{6,7}}$ and tissue inhibitor of metalloproteinase-1 (TIMP-1) ${ }^{6}$ with micro- and macrovascular complications in individuals with type 1 diabetes. In addition, we recently showed significant associations, in type 1 diabetes, of higher plasma levels of MMP-2 with incident cardiovascular disease (CVD) and of higher plasma levels of MMP-1, -2 and -3 with all-cause mortality. ${ }^{8}$ We hypothesized that AGEs and the MMP-TIMP system are associated and thereby may contribute to vascular complications in individuals with type 1 diabetes. Evidence so far with regard to the effect of AGEs on MMP production has shown mixed results. On the one hand, several AGEs have been shown to induce the expression of MMP-1, ${ }^{9}$ MMP-2, ${ }^{3,10-16}$ MMP-9, ${ }^{9,10,12-14,16-22}$ MMP-13 $3^{14}$ and TIMP-1 ${ }^{23}$ in various cell types associated with vascular complications like retinal cells, ${ }^{10,11,20}$ mesangial cells, ${ }^{16,23}$ endothelial cells, ${ }^{12,13}$ renal tubular cells, ${ }^{3}$ fibroblasts ${ }^{14,18}$ and macrophages. ${ }^{22}$ This mechanism seems to be mediated, at least partly, by RAGE. However, other studies have demonstrated no effect of AGEs on the expression of MMP-2, ${ }^{9,19,24-26} \mathrm{MMP}^{2},{ }^{27} \mathrm{MMP}^{2} \mathrm{9}^{15}$ and MMP-10 ${ }^{27}$ in keratinocytes, ${ }^{19}$ monocytes, $^{9}$ mesangial cells ${ }^{25,27}$ and endothelial cells. ${ }^{15,26}$ In addition, decreases in MMP-7 ${ }^{27}$ and TIMP-1 ${ }^{19}$ have also been observed after stimulation by AGEs. Notwithstanding these data from experimental work, data on the associations between circulating specific AGEs and a panel of MMPs and TIMP-1 in individuals with type 1 diabetes are lacking.

Therefore, we have investigated whether circulating levels of CML, CEL, MG-H1, and pentosidine are associated with circulating levels of MMP-1, -2, -3, -9, and -10, and TIMP-1 in patients with type 1 diabetes. 


\section{Methods}

\section{Study population}

The PROFIL study is a cross-sectional study that was conducted at the Steno Diabetes Center Copenhagen in Denmark, between 2009 and 2011, and included 676 individuals with type 1 diabetes; 316 patients were normoalbuminuric, 169 had microalbuminuria and 191 had macroalbuminuria. ${ }^{28}$ Patients with end stage renal disease, defined as receiving dialysis or renal transplantation, or estimated glomerular filtration rate (eGFR) $<15 \mathrm{~L} / \mathrm{min} / 1.73 \mathrm{~m}^{2}$ were excluded. The study was originally performed to investigate arterial stiffness and various diabetic complications. ${ }^{28}$ The study conformed to the Declaration of Helsinki and was approved by the Danish National Committee on Biomedical Research Ethics (2009-056; NCT01171248). All patients gave written informed consent. Individuals with missing data on AGEs, MMPs or TIMP-1 (n=6) were excluded from the present analyses, resulting in a total study population of 670 individuals.

\section{Main determinants}

We included four different AGEs in our analyses. On the basis of their characteristics each may represent a different part of the mechanisms through which AGEs could exert their effect on the expression of MMPs and TIMP-1. Thus, CML is a ligand of RAGE; CEL and MG-H1 are AGEs putatively derived from intracellular glycation by methylglyoxal (MGO); and pentosidine is a cross-link AGE. Serum protein-bound AGEs were measured from samples that were stored at $-80^{\circ} \mathrm{C}$ until analyses. For the measurement of the protein-bound $\mathrm{CML}$, CEL, MG-H1, and lysine an in-house method using (ultraperformance) liquid chromatography-tandem mass spectometry was used, which is described in detail elsewhere. ${ }^{29}$ Intra- and interassay coefficients of variation were $4.3 \%$ and $7.5 \%$ for $\mathrm{CML}, 5.9 \%$ and $4.5 \%$ for CEL, $9.8 \%$ and $11.5 \%$ for $\mathrm{MG}-\mathrm{H} 1$ and $4.3 \%$ and $7.6 \%$ for lysine. Protein-bound pentosidine was quantified using high-performance liquid chromatography with fluorescence detection, which is also described in detail elsewhere. ${ }^{30}$ Intra- and interassay coefficients of variation for pentosidine were $2.4 \%$ and $6.2 \%$. Concentrations of protein-bound $\mathrm{CML}, \mathrm{CEL}, \mathrm{MG}-\mathrm{H} 1$ and pentosidine were adjusted for levels of lysine and expressed as $\mathrm{nmol} / \mathrm{mmol}$ lysine.

\section{Main outcomes}

Concentrations of MMP-1, MMP-2, MMP-3, MMP-9, MMP-10 and TIMP-1 were determined in serum samples that were stored at $-80^{\circ} \mathrm{C}$ until analyses. Circulating levels of MMPs and TIMP-1 were measured using a commercially available multi-array enzymelinked immunosorbent assay (ELISA) kit [Human MMP-3-Plex Kit (for MMP-1, -3 and -9), Human MMP-2-Plex Kit (for MMP-2 and -10) and Human TIMP-1 Kit, MSD, Rockville, 
United States of America] according to the manufacturer's protocol. The MMPs were detected in both a pro- and an active form. TIMP-1 was detected only in the active form. The intra- and inter-assay coefficients of variation for MMP-1, -2, -3, -9, and -10 and TIMP-1 were $<10 \%$, except for the inter-assay coefficient of variation of MMP-1 (10.4\%).

\section{Potential confounders}

Demographic data and information about medication, duration of diabetes and use of antihypertensive medication were collected using questionnaires and patient records. After 15 minutes of supine rest, office BP was measured three times and averaged. Body weight and height were measured to calculate the body mass index (BMI). Blood samples were taken for measurements of total cholesterol, glycated hemoglobin $(\mathrm{HbA} 1 \mathrm{c})$ and creatinine. We estimated the GFR using the creatinine-based Chronic Kidney Disease Epidemiology Collaboration (CKD-EPI) equation. ${ }^{31}$ On the basis of standardized questionnaires, current users of $\geq 1$ cigarettes or cigars or pipes per day were classified as smokers and all others were classified as nonsmokers.

\section{Vascular complications}

Vascular complications were defined as presence of macro- and/or microvascular complications, i.e. CVD, albuminuria or retinopathy. Previous CVD was a history of myocardial infarction, coronary intervention, stroke, or peripheral arterial disease based on standardized World Health Organization questionnaires and patient records.

Urinary albumin excretion ratio (UAER) was measured in 24-h sterile urine collections by enzyme immunoassay. Patients were stratified as normoalbuminuric if they, in two out of three consecutive measurements, had persistent normoalbuminuria with UAER $<30 \mathrm{mg} / 24 \mathrm{~h}$ and were considered microalbuminuric or macroalbuminuric if UAER was between 30 and $300 \mathrm{mg} / 24 \mathrm{~h}$ or $>300 \mathrm{mg} / 24 \mathrm{~h}$, respectively. Retinopathy status was obtained from medical records. All patients attending Steno Diabetes Center have regular ophthalmological examinations, where retinopathy is assessed from retinal photographs taken through dilated pupils and graded as nil, simple, proliferative, or blind based on the worst eye.

\section{Statistical analysis}

All analyses were performed using the Statistical Package for Social Sciences (SPSS), version 20 (IBM Corporation, Armonk, NY, USA). Log-transformation was performed for variables with a skewed distribution (i.e. MMP-1, MMP-2, MMP-3, MMP-9, MMP-10 and TIMP-1). Linear regression analyses were applied to examine associations between circulating levels of $\mathrm{CML}, \mathrm{CEL}, \mathrm{MG}-\mathrm{H} 1$ and pentosidine on the one hand and circulating MMP-1, MMP-2, MMP-3, MMP-9, MMP-10 and TIMP-1 on the other. Analyses of these associations were adjusted for age, sex, $\mathrm{HbA} 1 \mathrm{c}$ and duration of diabetes (model 1 ). 
Further adjustments were made for total cholesterol, BMI, eGFR, smoking status, systolic blood pressure, diastolic blood pressure, use of antihypertensive medication, use of statins, and presence of any vascular complication (model 2). Results of these analyses are presented as difference in circulating InMMP or InTIMP-1 (in SD) per 1 SD higher level of circulating CML, CEL, MG-H1 and pentosidine.

We additionally tested for potential sex- and albuminuria status-associated interactions in the associations between $\mathrm{CML}, \mathrm{CEL}, \mathrm{MG}-\mathrm{H} 1$ and pentosidine on the one hand and MMPs and TIMP-1 on the other by adding a product term between sex or albuminuria status and $\mathrm{CML}, \mathrm{CEL}, \mathrm{MG}-\mathrm{H} 1$ and pentosidine to the fully adjusted model. The interaction analysis with albuminuria status was done to investigate whether or not oversampling of micro- and macroalbuminuric patients influenced the results. We stratified our analyses whenever significant interactions ( $p$ for interaction $<0.10$ ) were found.

\section{Results}

\section{Patient characteristics}

Baseline patient characteristics are shown in Table 5.1. Mean age was 54.6 years, 56\% were male and the mean duration of diabetes was 32.7 years. Seventy-two percent of the patients used antihypertensive medication and 60\% used statins. All MMPs, TIMP-1 and pentosidine levels showed a skewed distribution and are therefore presented as medians with inter-quartile ranges.

\section{Association between circulating AGEs and MMPs/TIMP-1}

The results of the linear regression analyses with adjustments for potential confounders are shown in Table 5.2. Circulating CML was positively associated with circulating MMP-2, MMP-3, and MMP-10 and inversely associated with MMP-9, after adjustment for age, sex, HbA1C and duration of diabetes (Table 5.2, model 1). After further adjustment for potential confounders, only the positive association of protein-bound CML with MMP-2 and the inverse association with MMP-9 remained significant $(\beta=0.11$ $(0.03 ; 0.19)$ and $\beta=-0.13(-0.22 ;-0.03)$, respectively (Table 5.2, model 2)).

After full adjustment, circulating CEL was positively associated with MMP-3 $[\beta=0.08$ $(0.02 ; 0.14)]$ and TIMP-1 $[\beta=0.08(0.01 ; 0.16)]$. The associations between CEL and MMP10 showed borderline significance after full adjustment $[\beta=0.07(0.00 ; 0.14)]$.

MG-H1 was significantly and positively associated with TIMP-1 after full adjustment $[\beta=0.09(0.02 ; 0.17)]$.

Circulating pentosidine was associated with MMP-1, MMP-2, MMP-3, MMP-10 and TIMP-1 in the minimally adjusted model (Table 5.2, model 1), but these associations did not remain significant after further adjustments (Table 5.2, model 2). 
Table $5.1 \quad$ Baseline characteristics of the 670 patients with type 1 diabetes

\begin{tabular}{lc}
\hline Age (years) & $54.6(12.6)$ \\
Sex (male/female, \%) & $56 / 44$ \\
BMI (kg/m²) & $25.3(4.0)$ \\
HbA1c (\%) & $8.0(1.2)$ \\
HbA1c (mmol/mol) & $64(12.7)$ \\
Duration of diabetes (years) & $32.7(15.8)$ \\
Total cholesterol (mmol/l) & $4.68(0.87)$ \\
Smoker (\%) & 20.7 \\
Systolic blood pressure (mmHg) & $132(18)$ \\
Diastolic blood pressure (mmHg) & $74(9)$ \\
Antihypertensive medication (\%) & 72 \\
Statin user (\%) & 60 \\
eGFR (ml/min/1.73m²) & $88[65-100]$ \\
Cardiovascular disease (\%) & 21.3 \\
Albuminuria (normo-/micro-/macro-, \%) & $47 / 25 / 28$ \\
Retinopathy (no/non-proliferative/proliferative/blind, \%) & $22 / 41 / 34 / 3$ \\
MMP-1 (ng/ml) & $20.9[12.8-30.9]$ \\
MMP-2 (ng/ml) & $142[128-159]$ \\
MMP-3 (ng/ml) & $19.2[12.8-28.5]$ \\
MMP-9 (ng/ml) & $160[107-240]$ \\
MMP-10 (pg/ml) & $1165[860-1679]$ \\
TIMP-1 (ng/ml) & $306[262-354]$ \\
CML (nmol/mmol lysine) & $101(26)$ \\
CEL (nmol/mmol lysine) & $30(11)$ \\
Pentosidine (nmol/mmol lysine) & $260(60)$ \\
\hline & $0.76[0.60-1.03]$ \\
\hline
\end{tabular}

Data are presented as means (standard deviation), median [inter-quartile range], or percentages, as appropriate. BMI, body mass index; HbA1c, glycated hemoglobin; eGFR, estimated glomerular filtration rate by CKD-EPI formula; MMP, matrix metalloproteinase; TIMP-1, tissue inhibitor of metalloproteinase-1; CML, $\mathrm{N}^{\varepsilon}$-(carboxymethyl)lysine; $\mathrm{CEL}, \mathrm{N}^{\varepsilon}$-(carboxyethyl)lysine; MG-H1, 5-hydro-5-methylimidazolone

\section{Additional analyses}

Adjustment for CVD, albuminuria and retinopathy separately did not materially change the results (data not shown). In addition, adjustment for RAAS inhibitors instead of antihypertensive therapy also did not change the results (data not shown). When we studied sex-associated differences in the fully adjusted model, the association between CML and MMP-2 was significant in women $[0.15(0.02 ; 0.29)]$, but not in men $[0.07$ $(-0.03 ; 0.17)]$ ( $p$ for interaction $=0.066)$. All other associations showed no sex-associated differences. In addition, we did not observe significant differences in the associations between AGEs and MMPs across the different albuminuria groups (data not shown, $p>0.10)$. 


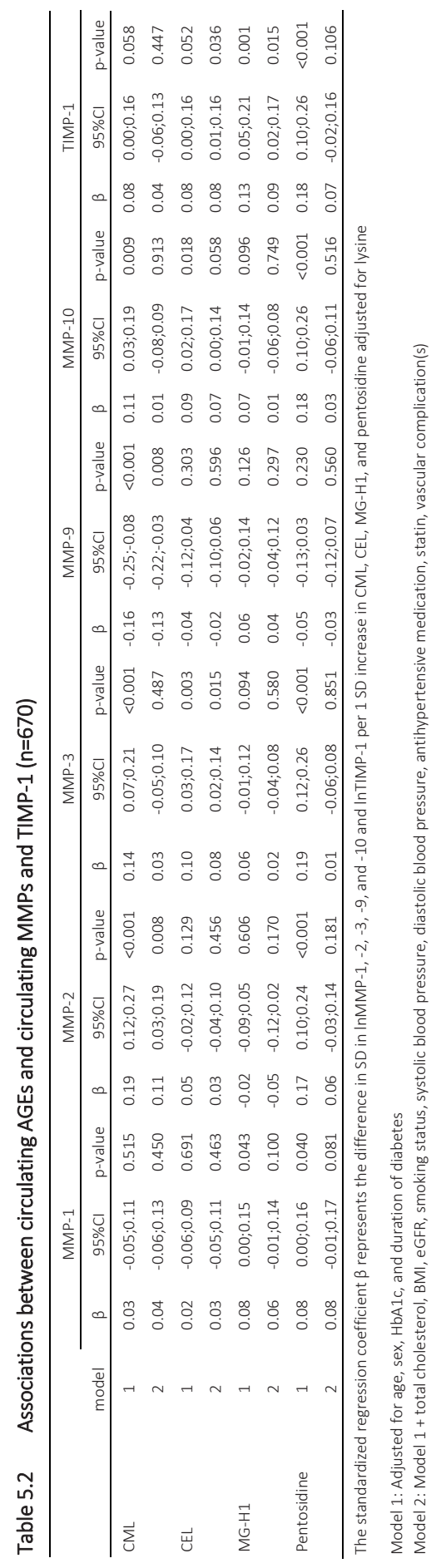




\section{Discussion}

In the present study, we showed that, in individuals with type 1 diabetes, circulating CML was positively associated with circulating MMP-2 and inversely associated with MMP-9. In addition, CEL was positively associated with MMP-3, and both CEL and MG-H1 were positively associated with TIMP-1. Pentosidine was not associated with any of the MMPS or TIMP-1. Oversampling of micro- and macroalbuminuric patients did not materially change the results as both adjustment for albuminuria status as well as interaction analysis did not materially change the results. To our knowledge, we are the first to show associations between circulating AGEs and circulating MMPs/TIMP-1 in patients with type 1 diabetes.

\section{CML and MMPs/TIMP-1}

The positive association between $\mathrm{CML}$ and MMP-2 is in line with previous findings in mouse mesangial cells ${ }^{16}$ as well as with an AGE-induced increase in MMP-2 in retinal cells, ${ }^{10-12}$ mesangial cells, ${ }^{25}$ endothelial cells ${ }^{13,15}$ and fibroblasts. ${ }^{14}$ In contrast, no AGEinduced MMP-2 production was observed in human keratinocytes, ${ }^{19}$ human monocytes ${ }^{9}$ and human umbilical cord endothelial cells ${ }^{26}$ Because the majority of the studies show that various cells in organs prone to cardiovascular complications seem potentially susceptible to AGE-mediated MMP-2 production, this pathway may therefore contribute to vascular complications.

In the current study, CML was inversely associated with MMP-9. This is in contrast to the study by Kim et al., who found that MMP-9 production increased in mouse mesangial cells after stimulation with CML. ${ }^{16}$ MMP-9 is one of the first MMPs to be produced after stimulation by e.g. hemodynamic forces, inflammatory cytokines, reactive oxygen species, hormonal stimuli and AGEs. ${ }^{12,32}$ The reasons for the discrepancy between our study and the study by Kim et al. ${ }^{16}$ as well as others who observed an AGE-mediated production of MMP-9 $9^{9,10,12-14,17-22}$ are not completely clear, but could in part be explained by the fact that in type 1 diabetes additional factors, such as TGF- $\beta,^{33}$ may influence the levels of MMP-9 and AGEs and as such the association between AGEs and MMPs.

We did not observe associations between CML and MMP-1, MMP-3, MMP-10 and TIMP-1. The findings of MMP-3 and MMP-10 are supported by the study by McLennan et al. $^{27}$ showing no effect of CML on the expression of MMP-3 and MMP-10 in human mesangial cells.

Overall, we showed that $\mathrm{CML}$, one of the major ligands for RAGE, ${ }^{34}$ is associated with altered expression of markers of the MMP/TIMP system. Further studies are needed to evaluate the exact role of RAGE in the association between $C M L$ and markers of the MMP/TIMP system and altered ECM remodeling. ${ }^{15,25}$ 


\section{CEL, MG-H1 and MMPs/TIMP-1}

Both CEL and MG-H1 are intracellular, MGO-derived AGEs. ${ }^{35}$ We showed that circulating levels of CEL were positively associated with both circulating MMP-3 and circulating TIMP-1. We are not aware of studies that investigated associations between CEL and MMP-3 and TIMP-1. Only McLennan et al. ${ }^{27}$ investigated an AGE-induced MMP-3 expression in human mesangial cells, but they observed no effect. In line with our study, Wang et al. ${ }^{23}$ showed an increase in TIMP-1 after stimulation of human renal mesangial cells by AGEs. In contrast, Zhu et al. ${ }^{19}$ showed, besides a significant increase in MMP-9, a significant decrease of mRNA levels of TIMP-1 after stimulation of human keratinocytes by AGE-BSA.

Besides CEL, MG-H1 was also positively associated with TIMP-1. Although the mechanism through which MGO-derived AGEs contribute to vascular complications is not fully understood, higher levels of MGO may decrease collagen turnover and induce collagen and TGF- $\beta$ expression. ${ }^{36}$ The latter is associated with an altered balance in the MMP-TIMP system leading to increase in TIMP-1 and inhibition of MMP activity. ${ }^{37}$ Additional studies need to be performed to evaluate the role MGO and MGO-derived AGEs of intracellular AGEs in production of MMPs and TIMPs.

\section{Pentosidine and MMPs/TIMP-1}

We did not find an association of pentosidine, a cross-linking extracellular AGE, with circulating MMPs or TIMP-1 after full adjustment for potential confounders. As far as we are aware, no other studies have reported on associations between circulating pentosidine and MMPs. In line with the current results, Furfaro et al. ${ }^{38}$ studied correlations between pentosidine and markers of ECM remodeling and inflammation in human advanced atheroslerotic plaques of individuals with diabetes and found a nonsignificant association between pentosidine content and MMP-2 expression. The lack of association between pentosidine and MMPs or TIMP-1 is not surprising since pentosidine forms cross-links between extracellular proteins and impairs their function, without a direct effect on protein synthesis.

\section{Limitations}

Firstly, we used serum levels of AGEs, MMPs and TIMP-1, because these can easily be obtained, but we do not know whether or not serum levels truly reflect tissue levels or if they originate from one or more specific tissues. Secondly, we cannot draw conclusions on pathophysiological mechanisms, because of the cross-sectional design of our study. 


\section{Conclusion}

We showed that serum levels of several AGEs representing different mechanisms through which AGEs exert effects were significantly associated with serum levels of several MMPs and/or TIMP-1. The results indicate that AGEs and the MMP-TIMP system are related and suggest distinct pathways for the relationships between individual AGEs and MMPs. Further studies are needed to further elucidate the potential mechanism by which AGEs influence the MMP/TIMP-1 system and thereby contribute to vascular complications in patients with type 1 diabetes. 


\section{References}

1. Nin JW, Jorsal A, Ferreira I, Schalkwijk CG, Prins MH, Parving HH, Tarnow L, Rossing P, Stehouwer CD: Higher plasma levels of advanced glycation end products are associated with incident cardiovascular disease and all-cause mortality in type 1 diabetes: a 12-year follow-up study. Diabetes Care 2011;34: 442-447

2. Brownlee M: Biochemistry and molecular cell biology of diabetic complications. Nature 2001;414: 813-820

3. Fukami K, Yamagishi S, Coughlan MT, Harcourt BE, Kantharidis P, Thallas-Bonke V, Okuda S, Cooper ME, Forbes JM: Ramipril inhibits AGE-RAGE-induced matrix metalloproteinase-2 activation in experimental diabetic nephropathy. Diabetol Metab Syndr 2014;6:86

4. McKittrick IB, Bogaert Y, Nadeau K, Snell-Bergeon J, Hull A, Jiang T, Wang X, Levi M, Moulton KS: Urinary matrix metalloproteinase activities: biomarkers for plaque angiogenesis and nephropathy in diabetes. Am J Physiol Renal Physiol 2011;301:F1326-1333

5. Derosa G, Avanzini MA, Geroldi D, Fogari R, Lorini R, De Silvestri A, Tinelli C, Rondini G, d'Annunzio G: Matrix metalloproteinase 2 may be a marker of microangiopathy in children and adolescents with type 1 diabetes mellitus. Diabetes Res Clin Pract 2005;70:119-125

6. Peeters SA, Engelen L, Buijs J, Chaturvedi N, Fuller JH, Schalkwijk CG, Stehouwer CD: Erratum to: Plasma levels of matrix metalloproteinase-2, $-3,-10$, and tissue inhibitor of metalloproteinase-1 are associated with vascular complications in patients with type 1 diabetes: the EURODIAB Prospective Complications Study. Cardiovasc Diabetol 2015;14:128

7. Toni M, Hermida J, Goni MJ, Fernandez P, Parks WC, Toledo E, Montes R, Diez N: Matrix metalloproteinase-10 plays an active role in microvascular complications in type 1 diabetic patients. Diabetologia 2013;56:2743-2752

8. Peeters SA, Engelen L, Buijs J, Jorsal A, Parving HH, Tarnow L, Rossing P, Schalkwijk CG, Stehouwer CDA: Plasma matrix metalloproteinases are associated with incident cardiovascular disease and all-cause mortality in patients with type 1 diabetes: a 12-year follow-up study. Cardiovasc Diabetol 2017;16:55

9. Bao W, Min D, Twigg SM, Shackel NA, Warner FJ, Yue DK, McLennan SV: Monocyte CD147 is induced by advanced glycation end products and high glucose concentration: possible role in diabetic complications. Am J Physiol Cell Physiol 2010;299:C1212-1219

10. Navaratna D, Menicucci G, Maestas J, Srinivasan R, McGuire P, Das A: A peptide inhibitor of the urokinase/urokinase receptor system inhibits alteration of the blood-retinal barrier in diabetes. Faseb J 2008;22:3310-3317

11. Sun Z, Liu J, Zeng X, Huangfu J, Jiang Y, Wang M, Chen F: Protective actions of microalgae against endogenous and exogenous advanced glycation endproducts (AGEs) in human retinal pigment epithelial cells. Food Funct 2011;2:251-258

12. Navaratna D, McGuire PG, Menicucci G, Das A: Proteolytic degradation of VE-cadherin alters the bloodretinal barrier in diabetes. Diabetes 2007;56:2380-2387

13. Luo P, Peng H, Li C, Ye Z, Tang H, Tang Y, Chen C, Lou T: Advanced glycation end products induce glomerular endothelial cell hyperpermeability by upregulating matrix metalloproteinase activity. Mol Med Rep 2015;11:4447-4453

14. Daoud S, Schinzel R, Neumann A, Loske C, Fraccarollo D, Diez C, Simm A: Advanced glycation endproducts: activators of cardiac remodeling in primary fibroblasts from adult rat hearts. Mol Med 2001; 7:543-551

15. Shimizu F, Sano Y, Tominaga O, Maeda T, Abe MA, Kanda T: Advanced glycation end-products disrupt the blood-brain barrier by stimulating the release of transforming growth factor-beta by pericytes and vascular endothelial growth factor and matrix metalloproteinase-2 by endothelial cells in vitro. Neurobiol Aging 2013;34:1902-1912

16. Kim KM, Jung DH, Jang DS, Kim YS, Kim JM, Kim HN, Surh YJ, Kim JS: Puerarin suppresses AGEs-induced inflammation in mouse mesangial cells: a possible pathway through the induction of heme oxygenase-1 expression. Toxicol Appl Pharmacol 2010;244:106-113

17. Zhu P, Yang C, Chen LH, Ren M, Lao GJ, Yan L: Impairment of human keratinocyte mobility and proliferation by advanced glycation end products-modified BSA. Arch Dermatol Res 2011;303:339-350 
18. Molinari J, Ruszova E, Velebny V, Robert L: Effect of advanced glycation endproducts on gene expression profiles of human dermal fibroblasts. Biogerontology 2008;9:177-182

19. Zhu P, Ren M, Yang C, Hu YX, Ran JM, Yan L: Involvement of RAGE, MAPK and NF-kappaB pathways in AGEs-induced MMP-9 activation in HaCaT keratinocytes. Exp Dermatol 2012;21:123-129

20. Chen YD, Xu X, Xia X, Wu H, Liu K, Zheng Z, Zhu D: MMP9 is involved in glycation end-products induced increase of retinal vascular permeability in rats and the therapeutic effect of minocycline. Curr Eye Res 2008;33:977-983

21. Lu W, Li J, Ren M, Zeng Y, Zhu P, Lin L, Lin D, Hao S, Gao Q, Liang J, Yan L, Yang C: Role of the mevalonate pathway in specific CpG site demethylation on AGEs-induced MMP9 expression and activation in keratinocytes. Mol Cell Endocrinol 2015;411:121-129

22. Zhang F, Banker G, Liu X, Suwanabol PA, Lengfeld J, Yamanouchi D, Kent KC, Liu B: The novel function of advanced glycation end products in regulation of MMP-9 production. J Surg Res 2011;171:871-876

23. Wang X, McLennan SV, Twigg SM: CCN-2 is up-regulated by and mediates effects of matrix bound advanced glycated end-products in human renal mesangial cells. J Cell Commun Signal 2011;5:193-200

24. Brings S, Zhang S, Choong YS, Hogl S, Middleditch M, Kamalov M, Brimble MA, Gong D, Cooper GJ: Diabetes-induced alterations in tissue collagen and carboxymethyllysine in rat kidneys: Association with increased collagen-degrading proteinases and amelioration by $\mathrm{Cu}(\mathrm{II})$-selective chelation. Biochim Biophys Acta 2015;1852:1610-1618

25. Berrou J, Tostivint I, Verrecchia F, Berthier C, Boulanger E, Mauviel A, Marti HP, Wautier MP, Wautier JL, Rondeau E, Hertig A: Advanced glycation end products regulate extracellular matrix protein and protease expression by human glomerular mesangial cells. Int J Mol Med 2009;23:513-520

26. Gharagozlian S, Henriksen T, Kolset SO: High glucose and Nepsilon-(carboxymethyl) lysine bovine serum albumin modulate release of matrix metalloproteinases in cultured human endothelial cells. Eur J Nutr 2006;45:283-290

27. McLennan SV, Kelly DJ, Schache M, Waltham M, Dy V, Langham RG, Yue DK, Gilbert RE: Advanced glycation end products decrease mesangial cell MMP-7: a role in matrix accumulation in diabetic nephropathy? Kidney Int 2007;72:481-488

28. Theilade S, Lajer M, Persson F, Joergensen C, Rossing P: Arterial stiffness is associated with cardiovascular, renal, retinal, and autonomic disease in type 1 diabetes. Diabetes Care 2013;36:715-721

29. Hanssen NM, Engelen L, Ferreira I, Scheijen JL, Huijberts MS, van Greevenbroek MM, van der Kallen CJ, Dekker JM, Nijpels G, Stehouwer CD, Schalkwijk CG: Plasma levels of advanced glycation endproducts Nepsilon-(carboxymethyl)lysine, Nepsilon-(carboxyethyl)lysine, and pentosidine are not independently associated with cardiovascular disease in individuals with or without type 2 diabetes: the Hoorn and CODAM studies. J Clin Endocrinol Metab 2013;98:E1369-1373

30. Scheijen JL, van de Waarenburg MP, Stehouwer CD, Schalkwijk CG: Measurement of pentosidine in human plasma protein by a single-column high-performance liquid chromatography method with fluorescence detection. J Chromatogr B Analyt Technol Biomed Life Sci 2009;877:610-614

31. Levey AS, Stevens LA, Schmid CH, Zhang YL, Castro AF, 3rd, Feldman HI, Kusek JW, Eggers P, Van Lente F, Greene T, Coresh J: A new equation to estimate glomerular filtration rate. Ann Intern Med 2009;150:604-612

32. Chen Q, Jin M, Yang F, Zhu J, Xiao Q, Zhang L: Matrix metalloproteinases: inflammatory regulators of cell behaviors in vascular formation and remodeling. Mediators Inflamm 2013;2013:928315

33. McLennan SV, Kelly DJ, Cox AJ, Cao Z, Lyons JG, Yue DK, Gilbert RE: Decreased matrix degradation in diabetic nephropathy: effects of ACE inhibition on the expression and activities of matrix metalloproteinases. Diabetologia 2002;45:268-275

34. Kislinger T, Fu C, Huber B, Qu W, Taguchi A, Du Yan S, Hofmann M, Yan SF, Pischetsrieder M, Stern D, Schmidt AM: N(epsilon)-(carboxymethyl)lysine adducts of proteins are ligands for receptor for advanced glycation end products that activate cell signaling pathways and modulate gene expression. J Biol Chem 1999;274:31740-31749

35. Maessen DE, Stehouwer CD, Schalkwijk CG: The role of methylglyoxal and the glyoxalase system in diabetes and other age-related diseases. Clin Sci (Lond) 2015;128:839-861

36. Monnier VM, Sun W, Gao X, Sell DR, Cleary PA, Lachin JM, Genuth S: Skin collagen advanced glycation endproducts (AGEs) and the long-term progression of sub-clinical cardiovascular disease in type 1 diabetes. Cardiovasc Diabetol 2015;14:118 
37. Xu X, Xiao L, Xiao P, Yang S, Chen G, Liu F, Kanwar YS, Sun L: A glimpse of matrix metalloproteinases in diabetic nephropathy. Curr Med Chem 2014;21:3244-3260

38. Furfaro AL, Sanguineti R, Storace D, Monacelli F, Puzzo A, Pronzato MA, Odetti P, Traverso N: Metalloproteinases and advanced glycation end products: coupled navigation in atherosclerotic plaque pathophysiology? Exp Clin Endocrinol Diabetes 2012;120:586-590 


\section{Chapter}

Summary and general discussion 



\section{Summary and general discussion}

As the number of patients with diabetes mellitus and its complications is increasing worldwide, more research about pathogenesis and prevention of the disease and its complications is desperately needed. The rising number is mainly caused by individuals with type 2 diabetes. However, also an increase in individuals with type 1 diabetes has been observed. In high-income countries about $7 \%$ to $12 \%$ of all diabetic patients have type 1 diabetes and the worldwide prevalence is increasing by $3 \%$ every year. ${ }^{1}$ The pathophysiological mechanisms leading to vascular complications (e.g. cardiovascular disease (CVD), albuminuria and retinopathy) have not been fully elucidated.

To date, altered extracellular matrix (ECM) remodeling has been extensively investigated in animal models of type 1 diabetes. ${ }^{2-18}$ In contrast, studies investigating associations between altered ECM remodeling in humans with type 1 diabetes are scarce, inconsistent and have various limitations, like small study groups and minimal adjustments for potential confounders. ${ }^{19-25}$ The lack of robust studies in humans has stimulated us to investigate the role of ECM remodeling in vascular complications. In this thesis we studied patients with type 1 diabetes included in three large study cohorts (EURODIAB, LEACE and PROFIL). Serum and plasma samples were used to evaluate circulating levels of markers of the matrix metalloproteinase (MMP) - tissue inhibitor of metalloproteinase (TIMP) system and associations with complications were investigated using the original data provided. In this section, the key findings of the four studies are summarized and discussed in light of the current knowledge of ECM remodeling by MMPs and TIMP-1, enzymes fundamentally involved in both vascular collagen and elastin proteolysis, in type 1 diabetes.

\section{MMPs, TIMP-1 and vascular complications}

In chapter two, a positive association is described between serum levels of TIMP-1 and prior CVD in the cross-sectional nested case-control EURODIAB study including 493 type 1 diabetic individuals. Although the association between circulating TIMP-1 and prior CVD was observed in two studies with individuals with type 2 diabetes and a history of coronary artery disease, ${ }^{26,27}$ we were the first who published the association of TIMP-1 with prior CVD in type 1 diabetes. Higher levels of TIMP-1 may reflect arterial remodeling after myocardial infarction, but can also be a marker of (post-myocardial infarction) cardiac fibrosis, potentially combined with decreased left ventricular function. ${ }^{2,28}$ Unfortunately, we do not have data on left ventricular function to evaluate whether or not higher serum TIMP-1 levels are also attributable to altered ventricular function.

In addition, we showed that serum levels of MMP-2, -3, -10 and TIMP-1 were positively associated with macroalbuminuria, whereas only MMP-2 was positively associated with microalbuminuria. Similar cross-sectional analyses in the prospective LEACE study $(n=370)$ support these results. MMP-2 and TIMP-1 were also associated with macroalbuminuria after full adjustment for potential confounders (unpublished data). 
Moreover, analyses in the cross-sectional PROFIL study ( $n=638$ ) showed similar associations between MMPs and TIMP-1 and macroalbuminuria as in the EURODIAB study (unpublished data). Variation of these results may be explicable by a smaller number of patients in the LEACE study, differences in presence of vascular complications between the studies and by the fact that in EURODIAB and PROFIL serum samples and in LEACE plasma samples were used for the measurements. Differences between serum and plasma measurements of MMPs and TIMP-1 will be discussed in the methodological considerations section. Our findings are supported by previous studies that also observed associations between MMP- $2,^{29} \mathrm{MMP}^{2}{ }^{30}{ }^{30} \mathrm{MMP}-10^{20}$ and $\mathrm{TIMP}^{2}{ }^{3}$ and albuminuria in animal models of diabetes and in humans. These results indicate that these MMPs and TIMP-1 may provide therapeutical targets to prevent (deterioration of) diabetic kidney disease.

In chapter two, we also showed the cross-sectional association between circulating MMP-2 and proliferative retinopathy. MMP-2 is overall one of the most studied MMPs in diabetic retinopathy and other studies also observed clear associations between MMP-2 and retinopathy. ${ }^{4,15-17,24}$ MMP-2 is one of the main MMPs that degrade type IV collagen, which is present in glomerular and retinal basement membranes, and tight junction proteins. Higher MMP-2 activity can lead to increased remodeling and increased permeability $^{4,15-17}$ in both tissues. In addition to albuminuria, MMP-2 might provide a target to prevent proliferative retinopathy.

Although circulating and tissue levels of MMP-9 have been associated with both diabetic nephropathy $y^{8,9,12,31}$ and retinopathy, ${ }^{15,18,24}$ we did not observe these associations in all three studies. This discrepancy might be caused by different stages of disease. In addition, MMP-9 may be produced mainly at the initial phase of ECM remodeling after which its significant role diminishes during the process of remodeling and is taken over by other MMPs.

\section{MMPs, TIMP-1 and incident CVD and all-cause mortality}

In the third chapter, we showed that higher levels of MMP-2 at baseline were associated with incident CVD during a median follow-up of 12.3 years. In addition, higher baseline levels MMP-1, -2 and -3 were associated with increased all-cause mortality. Evidence regarding associations between markers of ECM remodeling and incident CVD in individuals with type 1 diabetes is not available, but results of several studies in nondiabetic individuals are in line with our finding. ${ }^{32-38}$ Mechanistically, MMP-2 is associated with plaque rupture, thrombus formation and vasoconstriction. ${ }^{33,37}$ Moreover, MMP-2 can lead to an increase of vascular fibrosis, decrease of elastin content and increase of vascular smooth muscle cell migration and may as such contribute to pathological vascular remodeling. ${ }^{32,38}$ A mechanism, although speculative, explaining our finding regarding levels of MMP-1 and all-cause mortality in individuals with type 1 diabetes is the degradation of collagen type I, II, III, VII, X and other matrix proteins. In addition, MMP-1 is associated with altered vascular remodeling, probably contributing to 
aneurysmatic vascular disease, as discussed in chapter 4. As MMP-3 has mainly the same substrates as MMP-2, we hypothesize that MMP-2 and MMP-3 contribute to remodeling in disease states through a similar mechanism.

\section{Mediation through estimated glomerular filtration rate}

Additional analyses showed that the associations between MMP-2 and incident CVD and between MMP-3 and all-cause mortality were attenuated after adjustment for estimated glomerular filtration rate (eGFR). We also showed that MMP-3, but not MMP2, was significantly associated with decrease in eGFR during follow-up. The nonsignificant association between MMP-2 and decrease in eGFR might be due to the relatively small number of patients $(n=317)$. In our opinion there are two possible explanations for the eGFR-mediated associations between MMPs and incident CVD/allcause mortality. Firstly, decreased eGFR might cause accumulation of circulating MMP-2 or MMP-3, contributing to incident CVD or all-cause mortality, respectively. Secondly, higher levels of MMP-2 or MMP-3 could contribute to decreased eGFR, which is a known risk factor for incident CVD and mortality. Unfortunately, the design of our study did not allow us to differentiate which pathway could have caused our findings.

\section{Low-grade inflammation and endothelial dysfunction}

In the studies presented in chapter two and three, the associations of MMPs with vascular complications were adjusted for markers of low-grade inflammation (LGI) and endothelial dysfunction (ED). We calculated z-scores for LGI and ED based on a panel of markers reflecting LGI and ED. ${ }^{39-41}$ Levels of MMPs are influenced by the inflammatory environment and may even be considered as markers of inflammation. However, we showed that our associations of MMPs with vascular complications remained after adjustment for markers of LGI and ED. Although we believe that we made well-reasoned scores of LGI and ED, we cannot exclude, however, that other markers of LGI and ED may influence our results and therefore we have to interpret our results with care.

\section{MMPs, TIMP-1 and markers of arterial stiffness}

In chapter four, we showed that MMP-1 was inversely associated with office pulse pressure (PP), whereas MMP-2 was positively associated with office PP. In addition, MMP-2 was also positively associated with brachial and central 24-h PP. Based on the structure of the thoracic aorta, it might be that MMP-1 degrades collagen type 1 in the thoracic aorta causing a decrease in collagen content of the vessel wall, leading to increased elasticity, decreased PP, and eventually to thoracic aortic aneurysm or dissection. ${ }^{42}$ In contrast, MMP-2 is known to degrade type IV collagen, present in the vascular basement membrane, and elastin. In addition, both MMP-2 and MMP-3 are able to increase matrix production by cleavage of tissue transforming growth factor- $\beta$ 
(TGF- $\beta$ ) and therefore stimulate a TGF- $\beta$-induced matrix production, ${ }^{43,44}$ mainly collagen and fibronectin. Based on this mechanism we expected that MMP-2 would also be associated with higher arterial stiffness, as observed with PP. Due to the amplification phenomenon, brachial PP may not truly represent central PP in young and healthy individuals. ${ }^{45}$ However, the accelerated arterial aging in type 1 diabetes has been previously proven. ${ }^{46}$ In addition, in a large study with type 1 diabetic individuals with a mean age of 39 years, brachial PP was positively associated with incident CVD during a median follow-up of 5.3 years. ${ }^{47}$ Therefore PP may be an adequate measurement for arterial stiffness at younger age in type 1 diabetic individuals.

In contrast to previous studies without individuals with type 1 diabetes, ${ }^{48-50}$ circulating MMP-2 was not associated with carotid-femoral pulse wave velocity (cfPWV). In contrast, circulating levels of MMP-3 were associated with cfPWV. In additional analysis we found that MMP-3 was associated with PP in individuals above the age of 40 . When we further analyzed the data we concluded that adjustment for both age and duration of diabetes may lead to overadjustment. Reanalysis of the data with only age as a potential confounder revealed a significant positive association between MMP-2 and cfPWV and the association between MMP-3 and cfPWV became even stronger. The intrinsic characteristics of MMP-2 and MMP-3, both largely sharing the same substrates and effect on matrix production, combined with the current literature give us plausible explanations for our findings. In addition, the difference in composition between thoracic and abdominal aorta could also indicate that MMP-3 is able to alter abdominal aortic tissue and to increase cfPWV. MMP-1 showed a borderline inverse association with cfPWV, which is largely in line with the finding between MMP-1 and PP.

\section{Advanced glycation endproducts and the MMP-TIMP system}

In the fifth chapter, we investigated associations between serum levels of advanced glycation endproducts (AGEs) and markers of the MMP-TIMP system. AGEs are involved in CVD in both type $1^{51}$ and type 2 diabetes. ${ }^{52}$ Literature provides lots of evidence that AGEs are able to induce MMP production..$^{29,53-66}$ In vitro studies have shown AGEinduced MMP expression in various cells that are associated with vascular complications ${ }^{29,57,62,65}$ We studied associations between circulating levels of proteinbound $N^{\varepsilon}$-(carboxymethyl)lysine (CML), $\quad N^{\varepsilon}$-(carboxyethyl)lysine (CEL), 5-hydro-5methylimidazolone (MG-H1), pentosidine and MMP-1, $-2,-3,-9$, and -10 , and TIMP-1. We showed that $\mathrm{CML}$, an extracellular AGE and a major ligand of the receptor of AGE (RAGE), was positively associated with circulating MMP-2 and inversely with MMP-9 after adjustment for potential confounders. CEL was positively associated with MMP-3 and TIMP-1 and MG-H1, together with CEL representing intracellular methylglyoxalderived AGEs, was only associated with TIMP-1. Circulating pentosidine, a cross-linking AGE, was not associated with circulating MMPs or TIMP-1.

Thus, our study showed several independent associations of several specific AGEs with specific MMPs or TIMP-1. Our findings indicate that CML is associated with increased 
matrix turnover, as indicated by the association with MMP-2. CML- and other AGEinduced MMP-2 expression have been published previously (chapter five). In contrast to $\mathrm{CML}$, the intracellular AGEs, CEL and MG-H1, might decrease matrix turnover, as shown by the associations with TIMP-1. As expected, the cross-link pentosidine showed no association with MMPs or TIMP-1.

Whereas literature largely showed positive associations between AGEs and MMPs/ TIMP-1, several explanations may clarify our findings of just a few associations between AGEs and markers of the MMP-TIMP system. Firstly, the contribution of AGEs to MMPmediated ECM remodeling may be overestimated despite current literature regarding AGE-mediated MMP and TIMP-1 production. Although various AGE stimulated cells have shown to induce MMP production, most of the experiments may have been performed with a concentration of AGEs higher than under physiological conditions. Therefore, we do not know if AGE stimulation at tissue level induces similar MMP production as compared to in vitro studies. In addition, at tissue level other factors, like e.g. growth factors and cytokines, may also alter MMP production. Finally, circulating markers of both MMPs and AGEs may not truly reflect the situation at tissue level and may underestimate the observed associations.

\section{Methodological considerations}

\section{Study populations}

This thesis comprises data of individuals with type 1 diabetes derived from three large studies: EURODIAB, LEACE and PROFIL. Patient characteristics of the three studies are shown in Supplemental Table S4.1.

From the EURODIAB study, we used the follow-up cross-sectional nested case-control study, which is a subset of 543 patients, re-evaluated seven years after initial inclusion. Patients with the largest vascular burden $(n=348)$ were compared to those without any vascular complication $(n=195)$. In the prospective LEACE study 391 type 1 diabetic individuals were included of whom 199 had diabetic nephropathy, defined as macroalbuminuria with a urinary albumin excretion (UAE) of $>300 \mathrm{mg} / 24 \mathrm{~h}$. The other 192 individuals were normoalbuminuric (UAE $<30 \mathrm{mg} / 24 \mathrm{~h}$ ). The median follow-up was 12.3 years and included data on incident CVD and all-cause mortality. Prospective analyses were performed in 337 individuals, whereas data regarding arterial stiffness was present in 370 subjects. The PROFIL study included 676 individuals with type 1 diabetes and 51 non-diabetic controls. The controls were included to investigate differences between them and patients with a short duration of diabetes. In the diabetes group, 316 patients were normoalbuminuric, 169 microalbuminuric and 191 had macroalbuminuria. So albuminuric patients were overrepresented by design. 
In our analyses, only patients with type 1 diabetes were included. The question remains to what extent the MMP-TIMP system acts similarly in the various types of diabetes and non-diabetic subjects.

A large number of potential confounders (e.g. age, sex, duration of diabetes, glycated hemoglobin A1c, eGFR, blood pressure, lipid profiles, body mass index, smoking status, antihypertensive medication and presence of vascular complications) were present in all studies and therefore we were able to adjust our associations for these confounders. However, we cannot rule out that other factors (e.g. growth factors) influenced our results. Finally, results from the EURODIAB and PROFIL study are only based on crosssectional analysis and therefore we can only speculate on causality in these studies.

\section{Markers of the MMP-TIMP system}

In all studies we determined the same markers of the MMP-TIMP system: MMP-1, MMP-2, MMP-3, MMP-9, MMP-10 and TIMP-1. We chose these markers based on the intrinsic characteristics of these enzymes that could contribute to vascular complications, as supported by current literature. For example, MMP-1 degrades collagen type $1{ }^{67}$ which is present in e.g. the aorta, and MMP-2, $-3,-9$ and -10 are known to degrade type IV collagen (as present in the basement membranes), fibronectin and elastin. ${ }^{67}$ So far, most studies investigated only one or two markers of the MMPTIMP system in type 1 diabetes. Furthermore, the few studies available on human subjects show several methodological shortcomings, like small sample size or incomplete adjustments for confounders. ${ }^{19-22}$ In our study, we had the ability to study a large panel of markers of the MMP-TIMP system in a large population of individuals with type 1 diabetes with a variety of vascular complications (CVD, retinopathy, nephropathy and arterial stiffness).

The absolute values of the various MMPs and TIMP-1 differ between the three studies, as shown in chapter four. The absolute concentrations of circulating levels in LEACE differ significantly from those in EURODIAB and PROFIL, which could be explained by the fact that analyses were performed in plasma samples in LEACE, whereas serum samples were used in the other studies. Indeed, higher levels of MMP-9 and TIMP-1 were observed in serum compared to plasma levels of the same individuals. ${ }^{68,69}$ In chapter four we used cohort-specific z-scores to overcome the absolute differences in concentrations of MMPs and TIMP-1 between plasma and serum levels and we made an additional adjustment for cohort.

Several other issues should be addressed:

As discussed in the first part of this discussion, we have shown various associations between MMPs and TIMP-1 and vascular complications. Surprisingly, MMP-9, one of the most studied MMPs, was not associated with vascular complications in our studies and this in contrast to several other studies, as mentioned in chapter two, three and four. Several explanations may be applicable to this finding. MMP-9 may be one of the first MMPs to be produced, inducing biochemical and/or structural alteration leading to 
vascular complications. In non-published data, we have seen in normoalbuminuric type 1 diabetic individuals that MMP-9 is associated with incident microalbuminuria after adjustment for potential confounders (LEACE study, $n=31$ ). This is in line with data published on type 2 diabetic individuals. ${ }^{31}$ It might be that MMP-9 precedes the production of other MMPs, after which MMP-9 levels decline. This may explain why we did not find associations between MMP-9 and established vascular complications in our subjects.

Samples from the EURODIAB and LEACE study were stored at -80 degrees Celsius and were approximately 16 years and 20 years old at the moment of analysis of our markers, respectively. It has been shown that plasma MMP-9 levels decrease even when stored at -80 degrees Celsius, ${ }^{70}$ while MMP-2 and TIMP-1 levels were not affected ${ }^{70}$ and we can not exclude the possibility that this may partly explain our observed lack of associations between MMP-9 and vascular complications. In addition, degradation after freeze-thaw cycles can influence the results too. MMP-2 levels are not influenced by repeated freeze-thaw cycles. ${ }^{71}$ However, pro-MMP-9 activity significantly decreases after 7 cycles, whereas MMP-9 activity does not decrease significantly. ${ }^{72}$ Because our samples have never been through more than five freeze-thaw cycles, we can exclude the possibility that degradation by freeze-thaw cycles has influenced our results.

Serum and plasma samples were used to measure MMPs and TIMP-1 as markers of ECM remodeling. Circulating leukocytes and platelets also produce MMPs and TIMPs ${ }^{73}$ and this may explain the differences observed between serum and plasma samples. ${ }^{68,73}$

Another important issue is that we do not know if circulating levels of the biomarkers really represent tissue levels. Therefore, we cannot state with certainty that the measured markers solely reflect altered remodeling. Our results, however, consistently show that largely the same MMPs and TIMP-1 are associated with markers of renal function (eGFR and UAE) in all three studies. Our theory on the roles of MMPs and TIMP1 in vascular complications, supported by the evidence that MMP-1, $-2,-3$, and -10 and TIMP-1 are associated with vascular complications in type 1 diabetes (chapter two, three and four), is also in line with current literature in animal ${ }^{3,4}$ and human studies. ${ }^{20,24,30}$ The above-mentioned points provide enough arguments that the previously mentioned concerns are not major issues and thus validate our results.

\section{Advanced glycation endproducts}

We also determined serum levels of several pre-specified AGEs. AGEs are a heterogeneous group of glucose-modified proteins and AGE-induced MMP expression has been published previously. ${ }^{29,53-66}$ We determined serum levels of $\mathrm{CML}, \mathrm{CEL}, \mathrm{MG}-\mathrm{H} 1$ and pentosidine in the PROFIL study and investigated associations between these AGEs and the markers of the MMP-TIMP system.

In addition to the remarks regarding the markers of the MMP-TIMP system, it might be that the circulating levels do not truly reflect tissue levels of AGEs. The AGEs represent markers of (cross-linking) extra- and intracellular AGEs ${ }^{74}$ and the question rises whether 
or not these markers can easily translocate to the circulation. It might be that this underestimates our findings and a careful interpretation of these results is needed. To elucidate possible associations between AGEs and markers of the MMP-TIMP system further studies are needed.

\section{Future perspectives}

In this thesis we have shown cross-sectional and prospective associations between circulating markers of the MMP-TIMP system and vascular complications in individuals with type 1 diabetes. These results indicate that altered ECM remodeling is a key feature in vascular complications in individuals with type 1 diabetes. Since we have measured only a selection of markers of the family of MMPs and TIMPs, additional studies are needed to further elucidate whether or not other MMPs and/or TIMPs are associated with vascular complications. Moreover, influences of TGF- $\beta$ and other growth hormones should be evaluated, as both matrix degradation/turnover, but also matrix production/accumulation are associated with vascular complications. ${ }^{43,44}$

Several studies have demonstrated that pathological remodeling can be attenuated if current treatment options are provided or intensified. Intensive glucose treatment ${ }^{75}$ and blockade the renin-angiotensin-aldosterone system ${ }^{12,13,29}$ significantly contribute to restoring the balance between MMPs and TIMPs and (partly) attenuate pathological ECM remodeling. In addition, broad-spectrum and specific MMP-inhibitors have been developed to prevent excess MMP-mediated ECM remodeling. ${ }^{76}$ Moreover, also antibodies against membrane-type MMPs, which are involved in the activation of MMPs, are available. These antibodies prevent MMP transformation from the inactive to the active form. ${ }^{76}$ Statins, bisphosphonates, thiazolidinediones, aspirin and other COXinhibitors have shown to suppress MMP-production through anti-inflammatory mechanisms. $^{15,76}$ Tetracyclines also alter MMP-activity. ${ }^{15,62,76,77}$ Further studies are needed to investigate the potential use of these different treatment strategies in order to prevent MMP-mediated ECM remodeling in clinical practice. Some of these medicines are already frequently used, so studies on their effects can be obtained in clinical studies. Furthermore, we question whether development of specific MMP-inhibiting agents will be a panacea in preventing vascular complications.

In conclusion, this thesis highlights associations between markers of the MMP-TIMP system and vascular complications in individuals with type 1 diabetes. These crosssectional and prospective associations show that certain markers of this system may be suitable as therapeutical targets to treat or prevent vascular injury in patients with type 1 diabetes. 


\section{References}

1. IDF Diabetes Atlas 2015

2. Lu L, Zhang Q, Pu LJ, Peng WH, Yan XX, Wang L, Chen QJ, Zhu ZB, Michel JB, Shen WF: Dysregulation of matrix metalloproteinases and their tissue inhibitors is related to abnormality of left ventricular geometry and function in streptozotocin-induced diabetic minipigs. Int J Exp Pathol 2008;89:125-137

3. Alter ML, Kretschmer A, Von Websky K, Tsuprykov O, Reichetzeder C, Simon A, Stasch JP, Hocher B: Early urinary and plasma biomarkers for experimental diabetic nephropathy. Clin Lab 2012;58:659-671

4. Mohammad G, Kowluru RA: Matrix metalloproteinase-2 in the development of diabetic retinopathy and mitochondrial dysfunction. Lab Invest 2010;90:1365-1372

5. Eguchi M, Xu G, Li RK, Sweeney G: Diabetes influences cardiac extracellular matrix remodelling after myocardial infarction and subsequent development of cardiac dysfunction. J Cell Mol Med 2012;16:2925-2934

6. Mohammadzadeh F, Desjardins JF, Tsoporis JN, Proteau G, Leong-Poi H, Parker TG: S100B: role in cardiac remodeling and function following myocardial infarction in diabetes. Life Sci 2013;92:639-647

7. Dixon A, Maric C: 17beta-Estradiol attenuates diabetic kidney disease by regulating extracellular matrix and transforming growth factor-beta protein expression and signaling. Am J Physiol Renal Physiol 2007;293:F1678-1690

8. Mankhey RW, Wells CC, Bhatti F, Maric C: 17beta-Estradiol supplementation reduces tubulointerstitial fibrosis by increasing MMP activity in the diabetic kidney. Am J Physiol Regul Integr Comp Physiol 2007;292:R769-777

9. Takamiya Y, Fukami K, Yamagishi S, Kaida Y, Nakayama Y, Obara N, Iwatani R, Ando R, Koike K, Matsui T, Nishino Y, Ueda S, Cooper ME, Okuda S: Experimental diabetic nephropathy is accelerated in matrix metalloproteinase-2 knockout mice. Nephrol Dial Transplant 2013;28:55-62

10. Maric-Bilkan C, Flynn ER, Chade AR: Microvascular disease precedes the decline in renal function in the streptozotocin-induced diabetic rat. Am J Physiol Renal Physiol 2012;302:F308-315

11. Liu DX, Liu XM, Su Y, Zhang XJ: Renal expression of proto-oncogene Ets-1 on matrix remodeling in experimental diabetic nephropathy. Acta Histochem 2011;113:527-533

12. McLennan SV, Kelly DJ, Cox AJ, Cao Z, Lyons JG, Yue DK, Gilbert RE: Decreased matrix degradation in diabetic nephropathy: effects of ACE inhibition on the expression and activities of matrix metalloproteinases. Diabetologia 2002;45:268-275

13. Sun SZ, Wang Y, Li Q, Tian YJ, Liu MH, Yu YH: Effects of benazepril on renal function and kidney expression of matrix metalloproteinase-2 and tissue inhibitor of metalloproteinase-2 in diabetic rats. Chin Med J (Engl) 2006;119:814-821

14. Shankland SJ, Ly H, Thai K, Scholey JW: Glomerular expression of tissue inhibitor of metalloproteinase (TIMP-1) in normal and diabetic rats. J Am Soc Nephrol 1996;7:97-104

15. Bhatt LK, Addepalli V: Attenuation of diabetic retinopathy by enhanced inhibition of MMP-2 and MMP-9 using aspirin and minocycline in streptozotocin-diabetic rats. Am J Transl Res 2010;2:181-189

16. Mohammad G, Kowluru RA: Novel role of mitochondrial matrix metalloproteinase-2 in the development of diabetic retinopathy. Invest Ophthalmol Vis Sci 2011;52:3832-3841

17. Kowluru RA, Kanwar M: Oxidative stress and the development of diabetic retinopathy: contributory role of matrix metalloproteinase-2. Free Radic Biol Med 2009;46:1677-1685

18. Kowluru RA: Role of matrix metalloproteinase-9 in the development of diabetic retinopathy and its regulation by H-Ras. Invest Ophthalmol Vis Sci 2010;51:4320-4326

19. Thrailkill KM, Bunn RC, Moreau CS, Cockrell GE, Simpson PM, Coleman HN, Frindik JP, Kemp SF, Fowlkes JL: Matrix metalloproteinase-2 dysregulation in type 1 diabetes. Diabetes Care 2007;30:2321-2326

20. Toni M, Hermida J, Goni MJ, Fernandez P, Parks WC, Toledo E, Montes R, Diez N: Matrix metalloproteinase-10 plays an active role in microvascular complications in type 1 diabetic patients. Diabetologia 2013;56:2743-2752

21. Gharagozlian S, Svennevig K, Bangstad HJ, Winberg JO, Kolset SO: Matrix metalloproteinases in subjects with type 1 diabetes. BMC Clin Pathol 2009;9:7 
22. Jacqueminet S, Ben Abdesselam O, Chapman MJ, Nicolay N, Foglietti MJ, Grimaldi A, Beaudeux JL: Elevated circulating levels of matrix metalloproteinase- 9 in type 1 diabetic patients with and without retinopathy. Clin Chim Acta 2006;367:103-107

23. Thrailkill KM, Moreau CS, Cockrell GE, Jo CH, Bunn RC, Morales-Pozzo AE, Lumpkin CK, Fowlkes JL: Disease and gender-specific dysregulation of NGAL and MMP-9 in type 1 diabetes mellitus. Endocrine 2010;37:336-343

24. Salzmann J, Limb GA, Khaw PT, Gregor ZJ, Webster L, Chignell AH, Charteris DG: Matrix metalloproteinases and their natural inhibitors in fibrovascular membranes of proliferative diabetic retinopathy. Br J Ophthalmol 2000;84:1091-1096

25. Suzuki D, Yagame M, Kim Y, Sakai H, Mauer M: Renal in situ hybridization studies of extracellular matrix related molecules in type 1 diabetes mellitus. Nephron 2002;92:564-572

26. Opstad TB, Pettersen AA, Weiss TW, Akra S, Ovstebo R, Arnesen H, Seljeflot I: Genetic variation, geneexpression and circulating levels of matrix metalloproteinase- 9 in patients with stable coronary artery disease. Clin Chim Acta 2012;413:113-120

27. Derosa G, Cicero AF, Scalise F, Avanzini MA, Tinelli C, Peros E, Fogari E, D'Angelo A: Metalloproteinases in diabetics and nondiabetics during acute coronary syndromes and after 3 months. Endothelium 2007; 14:175-183

28. Kelly D, Khan SQ, Thompson M, Cockerill G, Ng LL, Samani N, Squire IB: Plasma tissue inhibitor of metalloproteinase-1 and matrix metalloproteinase-9: novel indicators of left ventricular remodelling and prognosis after acute myocardial infarction. Eur Heart J 2008;29:2116-2124

29. Fukami K, Yamagishi S, Coughlan MT, Harcourt BE, Kantharidis P, Thallas-Bonke V, Okuda S, Cooper ME, Forbes JM: Ramipril inhibits AGE-RAGE-induced matrix metalloproteinase-2 activation in experimental diabetic nephropathy. Diabetol Metab Syndr 2014;6:86

30. Suzuki D, Miyazaki M, Jinde K, Koji T, Yagame M, Endoh M, Nomoto Y, Sakai H: In situ hybridization studies of matrix metalloproteinase-3, tissue inhibitor of metalloproteinase-1 and type IV collagen in diabetic nephropathy. Kidney Int 1997;52:111-119

31. Ebihara I, Nakamura T, Shimada N, Koide H: Increased plasma metalloproteinase-9 concentrations precede development of microalbuminuria in non-insulin-dependent diabetes mellitus. Am J Kidney Dis 1998;32:544-550

32. Chen Q, Jin M, Yang F, Zhu J, Xiao Q, Zhang L: Matrix metalloproteinases: inflammatory regulators of cell behaviors in vascular formation and remodeling. Mediators Inflamm 2013;2013:928315

33. Gresele P, Falcinelli E, Loffredo F, Cimmino G, Corazzi T, Forte L, Guglielmini G, Momi S, Golino P: Platelets release matrix metalloproteinase-2 in the coronary circulation of patients with acute coronary syndromes: possible role in sustained platelet activation. Eur Heart J 2011;32:316-325

34. Ehrlich JR, Kaluzny M, Baumann S, Lehmann R, Hohnloser SH: Biomarkers of structural remodelling and endothelial dysfunction for prediction of cardiovascular events or death in patients with atrial fibrillation. Clin Res Cardiol 2011;100:1029-1036

35. Perez-Hernandez N, Vargas-Alarcon G, Martinez-Rodriguez N, Martinez-Rios MA, Pena-Duque MA, PenaDiaz Ade L, Valente-Acosta B, Posadas-Romero C, Medina A, Rodriguez-Perez JM: The matrix metalloproteinase 2-1575 gene polymorphism is associated with the risk of developing myocardial infarction in Mexican patients. J Atheroscler Thromb 2012;19:718-727

36. Nilsson L, Hallen J, Atar D, Jonasson L, Swahn E: Early measurements of plasma matrix metalloproteinase-2 predict infarct size and ventricular dysfunction in ST-elevation myocardial infarction. Heart 2012;98:31-36

37. Berry E, Bosonea AM, Wang X, Fernandez-Patron C: Insights into the activity, differential expression, mutual regulation, and functions of matrix metalloproteinases and a disintegrin and metalloproteinases in hypertension and cardiac disease. J Vasc Res 2013;50:52-68

38. Lan TH, Huang XQ, Tan HM: Vascular fibrosis in atherosclerosis. Cardiovasc Pathol 2013;22:401-407

39. Schram MT, Chaturvedi N, Schalkwijk C, Giorgino F, Ebeling P, Fuller JH, Stehouwer CD: Vascular risk factors and markers of endothelial function as determinants of inflammatory markers in type 1 diabetes: the EURODIAB Prospective Complications Study. Diabetes Care 2003;26:2165-2173

40. Schram MT, Chaturvedi N, Schalkwijk CG, Fuller JH, Stehouwer CD: Markers of inflammation are crosssectionally associated with microvascular complications and cardiovascular disease in type 1 diabetes-the EURODIAB Prospective Complications Study. Diabetologia 2005;48:370-378 
41. Soedamah-Muthu SS, Chaturvedi N, Schalkwijk CG, Stehouwer CD, Ebeling P, Fuller JH: Soluble vascular cell adhesion molecule-1 and soluble E-selectin are associated with micro- and macrovascular complications in Type 1 diabetic patients. J Diabetes Complications 2006;20:188-195

42. Koullias GJ, Ravichandran P, Korkolis DP, Rimm DL, Elefteriades JA: Increased tissue microarray matrix metalloproteinase expression favors proteolysis in thoracic aortic aneurysms and dissections. Ann Thorac Surg 2004;78:2106-2110; discussion 2110-2101

43. Imai K, Hiramatsu A, Fukushima D, Pierschbacher MD, Okada Y: Degradation of decorin by matrix metalloproteinases: identification of the cleavage sites, kinetic analyses and transforming growth factorbeta1 release. Biochem J 1997;322 ( Pt 3):809-814

44. Ruiz-Ortega M, Rodriguez-Vita J, Sanchez-Lopez E, Carvajal G, Egido J: TGF-beta signaling in vascular fibrosis. Cardiovasc Res 2007; 74:196-206

45. Laurent S, Cockcroft J, Van Bortel L, Boutouyrie P, Giannattasio C, Hayoz D, Pannier B, Vlachopoulos C, Wilkinson I, Struijker-Boudier H, European Network for Non-invasive Investigation of Large A: Expert consensus document on arterial stiffness: methodological issues and clinical applications. Eur Heart J 2006;27:2588-2605

46. Ronnback M, Fagerudd J, Forsblom C, Pettersson-Fernholm K, Reunanen A, Groop PH, Finnish Diabetic Nephropathy Study G: Altered age-related blood pressure pattern in type 1 diabetes. Circulation 2004;110:1076-1082

47. Gordin D, Waden J, Forsblom C, Thorn L, Rosengard-Barlund M, Tolonen N, Saraheimo M, Harjutsalo V, Groop PH, FinnDiane Study G: Pulse pressure predicts incident cardiovascular disease but not diabetic nephropathy in patients with type 1 diabetes (The FinnDiane Study). Diabetes Care 2011;34:886-891

48. Yasmin, McEniery CM, Wallace S, Dakham Z, Pulsalkar P, Maki-Petaja K, Ashby MJ, Cockcroft JR, Wilkinson IB: Matrix metalloproteinase-9 (MMP-9), MMP-2, and serum elastase activity are associated with systolic hypertension and arterial stiffness. Arterioscler Thromb Vasc Biol 2005;25:372

49. Tzemos N, Lyseggen E, Silversides C, Jamorski M, Tong JH, Harvey P, Floras J, Siu S: Endothelial function, carotid-femoral stiffness, and plasma matrix metalloproteinase-2 in men with bicuspid aortic valve and dilated aorta. J Am Coll Cardiol 2010;55:660-668

50. Smith ER, Tomlinson LA, Ford ML, McMahon LP, Rajkumar C, Holt SG: Elastin degradation is associated with progressive aortic stiffening and all-cause mortality in predialysis chronic kidney disease. Hypertension 2012;59:973-978

51. Nin JW, Jorsal A, Ferreira I, Schalkwijk CG, Prins MH, Parving HH, Tarnow L, Rossing P, Stehouwer CD: Higher plasma levels of advanced glycation end products are associated with incident cardiovascular disease and all-cause mortality in type 1 diabetes: a 12-year follow-up study. Diabetes Care 2011;34: 442-447

52. Hanssen NM, Beulens JW, van Dieren S, Scheijen JL, van der AD, Spijkerman AM, van der Schouw YT, Stehouwer CD, Schalkwijk CG: Plasma advanced glycation end products are associated with incident cardiovascular events in individuals with type 2 diabetes: a case-cohort study with a median follow-up of 10 years (EPIC-NL). Diabetes 2015;64:257-265

53. Navaratna D, Menicucci G, Maestas J, Srinivasan R, McGuire P, Das A: A peptide inhibitor of the urokinase/urokinase receptor system inhibits alteration of the blood-retinal barrier in diabetes. Faseb J 2008;22:3310-3317

54. Zhu P, Yang C, Chen LH, Ren M, Lao GJ, Yan L: Impairment of human keratinocyte mobility and proliferation by advanced glycation end products-modified BSA. Arch Dermatol Res 2011;303:339-350

55. Sun Z, Liu J, Zeng X, Huangfu J, Jiang Y, Wang M, Chen F: Protective actions of microalgae against endogenous and exogenous advanced glycation endproducts (AGEs) in human retinal pigment epithelial cells. Food Funct 2011;2:251-258

56. Navaratna D, McGuire PG, Menicucci G, Das A: Proteolytic degradation of VE-cadherin alters the bloodretinal barrier in diabetes. Diabetes 2007;56:2380-2387

57. Luo P, Peng H, Li C, Ye Z, Tang H, Tang Y, Chen C, Lou T: Advanced glycation end products induce glomerular endothelial cell hyperpermeability by upregulating matrix metalloproteinase activity. Mol Med Rep 2015;11:4447-4453

58. Daoud S, Schinzel R, Neumann A, Loske C, Fraccarollo D, Diez C, Simm A: Advanced glycation endproducts: activators of cardiac remodeling in primary fibroblasts from adult rat hearts. Mol Med $2001 ; 7: 543-551$ 
59. Shimizu F, Sano Y, Tominaga O, Maeda T, Abe MA, Kanda T: Advanced glycation end-products disrupt the blood-brain barrier by stimulating the release of transforming growth factor-beta by pericytes and vascular endothelial growth factor and matrix metalloproteinase-2 by endothelial cells in vitro. Neurobiol Aging 2013;34:1902-1912

60. Molinari J, Ruszova E, Velebny V, Robert L: Effect of advanced glycation endproducts on gene expression profiles of human dermal fibroblasts. Biogerontology 2008;9:177-182

61. Zhu P, Ren M, Yang C, Hu YX, Ran JM, Yan L: Involvement of RAGE, MAPK and NF-kappaB pathways in AGEs-induced MMP-9 activation in HaCaT keratinocytes. Exp Dermatol 2012;21:123-129

62. Chen YD, Xu X, Xia X, Wu H, Liu K, Zheng Z, Zhu D: MMP9 is involved in glycation end-products induced increase of retinal vascular permeability in rats and the therapeutic effect of minocycline. Curr Eye Res 2008;33:977-983

63. Bao W, Min D, Twigg SM, Shackel NA, Warner FJ, Yue DK, McLennan SV: Monocyte CD147 is induced by advanced glycation end products and high glucose concentration: possible role in diabetic complications. Am J Physiol Cell Physiol 2010;299:C1212-1219

64. Lu W, Li J, Ren M, Zeng Y, Zhu P, Lin L, Lin D, Hao S, Gao Q, Liang J, Yan L, Yang C: Role of the mevalonate pathway in specific CPG site demethylation on AGEs-induced MMP9 expression and activation in keratinocytes. Mol Cell Endocrinol 2015;411:121-129

65. Kim KM, Jung DH, Jang DS, Kim YS, Kim JM, Kim HN, Surh YJ, Kim JS: Puerarin suppresses AGEs-induced inflammation in mouse mesangial cells: a possible pathway through the induction of heme oxygenase-1 expression. Toxicol Appl Pharmacol 2010;244:106-113

66. Zhang F, Banker G, Liu X, Suwanabol PA, Lengfeld J, Yamanouchi D, Kent KC, Liu B: The novel function of advanced glycation end products in regulation of MMP-9 production. J Surg Res 2011;171:871-876

67. Nagase H, Visse R, Murphy G: Structure and function of matrix metalloproteinases and TIMPs. Cardiovasc Res 2006;69:562-573

68. Alby C, Ben Abdesselam O, Foglietti MJ, Beaudeux JL: Preanalytical aspects regarding the measurement of metalloproteinase- 9 and tissue inhibitor or metalloproteinase- 1 in blood. Clin Chim Acta 2002;325:183-186

69. Jung K, Gerlach RF, Tanus-Santos JE: Preanalytical pitfalls of blood sampling to measure true circulating matrix metalloproteinase 9 and tissue inhibitors of matrix metalloproteinases. Clin Chim Acta 2006;373:180-181; author reply 182

70. Rouy D, Ernens I, Jeanty C, Wagner DR: Plasma storage at -80 degrees C does not protect matrix metalloproteinase-9 from degradation. Anal Biochem 2005;338:294-298

71. Sulik A, Wojtkowska M, Oldak E: Preanalytical factors affecting the stability of matrix metalloproteinase-2 concentrations in cerebrospinal fluid. Clin Chim Acta 2008;392:73-75

72. Souza-Tarla CD, Uzuelli JA, Machado AA, Gerlach RF, Tanus-Santos JE: Methodological issues affecting the determination of plasma matrix metalloproteinase (MMP)-2 and MMP-9 activities. Clin Biochem 2005;38:410-414

73. Jung K, Lein $M$, Laube C, Lichtinghagen R: Blood specimen collection methods influence the concentration and the diagnostic validity of matrix metalloproteinase 9 in blood. Clin Chim Acta 2001;314:241-244

74. Brownlee M: Biochemistry and molecular cell biology of diabetic complications. Nature 2001;414: 813-820

75. Schuyler CA, Ta NN, Li Y, Lopes-Virella MF, Huang Y: Insulin treatment attenuates diabetes-increased atherosclerotic intimal lesions and matrix metalloproteinase 9 expression in apolipoprotein E-deficient mice. J Endocrinol 2011;210:37-46

76. Chistiakov DA, Sobenin IA, Orekhov AN: Vascular extracellular matrix in atherosclerosis. Cardiol Rev 2013;21:270-288

77. Franco C, Ho B, Mulholland D, Hou G, Islam M, Donaldson K, Bendeck MP: Doxycycline alters vascular smooth muscle cell adhesion, migration, and reorganization of fibrillar collagen matrices. Am J Pathol 2006;168:1697-1709 
Nederlandse samenvatting 



\section{Nederlandse samenvatting}

Diabetes mellitus, ook wel suikerziekte genoemd, is wereldwijd een toenemend probleem. In 2015 zijn er 415 miljoen diabetespatiënten in de wereld en dit aantal zal toenemen tot 642 miljoen in 2040. De hogere glucosewaarden in het bloed, die het kenmerk zijn van diabetes mellitus, kunnen op diverse manieren ontstaan. Bij type 1 diabetes mellitus, ongeveer 5 tot $10 \%$ van alle gevallen, ontstaat dit door afbraak van de insuline-producerende cellen in de alvleesklier, waardoor er een absoluut tekort aan insuline ontstaat. Bij type 2 diabetes mellitus is er sprake van verminderde insulinegevoeligheid op weefselniveau en zodoende is er sprake van een relatief insuline-tekort. In dit proefschrift hebben we ons gericht op patiënten met diabetes mellitus type 1 .

Aangezien diabetes mellitus vaker voor komt, komt ook een toename van de complicaties van de ziekte voor en voorbeelden hiervan zijn onder andere (o.a.) problemen met de bloedvaten (vasculaire complicaties).

Hieronder verstaan we:

1. problemen van de grotere bloedvaten die kunnen leiden tot een hartinfarct en/of cerebrovasculair accident (CVA)(macrovasculaire complicaties)

2. problemen van de kleinere bloedvaten in o.a. de ogen (retinopathie) en de nieren (nefropathie) (microvasculaire complicaties).

We weten ook dat het stijver zijn van de grootste slagader in het menselijk lichaam (de aorta) een risicofactor vormt op het krijgen van vasculaire complicaties.

Het optreden van deze complicaties kan leiden tot een verslechterde kwaliteit van leven en vroegtijdige sterfte. Het mechanisme dat leidt tot deze complicaties is nog niet geheel opgehelderd en aanpassingen in de samenstelling van een bloedvatwand is een van de problematische veranderingen die kan ontstaan. Echter is dit slechts onderzocht in met name dierenstudies en in enkele mensenstudies met patiënten met diabetes mellitus type 1.

Matrix metalloproteinasen (MMP's) zijn enzymen die betrokken zijn bij het natuurlijke herstel en aanpassingen van de extracellulaire matrix (ECM), o.a. de samenstelling van de bloedvatwand. Deze ECM zorgt voor ondersteuning tussen diverse cellen in een weefsel, maar ook tussen de cellen en het omliggende weefsel. De activiteit van deze MMP's is gereguleerd door o.a. natuurlijke remmers, tissue inhibitors of metalloproteinase (TIMP's). De activiteit van de MMP's kan ook worden verminderd door o.a. alfa-2-macroglobuline, bisfosfonaten, simvastatine en tetracyclines. Van de MMP's zijn er 24 bekend en daarnaast zijn er 4 TIMP's. Ontregeling van dit MMP-TIMP systeem kan leiden tot een veranderde samenstelling van de vaatwand en zodoende tot vasculaire complicaties.

In dit proefschrift hebben we gekeken naar associaties tussen circulerende waarden van MMP-1, -2, -3, -9 en -10 en TIMP-1 en vasculaire complicaties in patiënten met diabetes mellitus type 1 . Daarnaast hebben we associaties onderzocht tussen circulerende waarden van advanced glycation endproducts (AGE's, versuikerde eiwitten die ontstaan 
ten gevolge van blootstelling van eiwitten aan glucose) en circulerende MMP's. Er is namelijk aangetoond dat AGE's de MMP- en TIMP-productie kunnen beïnvloeden en zodoende kunnen AGE's bijdragen aan het ontstaan van veranderingen in de ECM.

\section{MMP's, TIMP-1 en vasculaire complicaties}

In hoofdstuk twee hebben we cross-sectionele associaties onderzocht tussen circulerende waarden van eerdergenoemde markers van ECM remodelering in 493 patiënten met diabetes mellitus type 1 met en zonder doorgemaakte hart- en vaatziekten (HVZ). We hebben laten zien dat mensen met doorgemaakt HVZ hogere spiegels van TIMP-1 hadden. Er waren geen associaties tussen HVZ en circulerende MMP's. We zijn de eersten die deze associatie hebben beschreven in patiënten met diabetes mellitus type 1 , maar deze associatie is wel eerder beschreven bij patiënten met diabetes mellitus type 2 .

Naast macrovasculaire complicaties hebben we ook gekeken naar microvasculaire complicaties (nier- en oogproblemen) in deze patiëntengroep. Eerst hebben we gekeken naar eiwituitscheiding in de urine (albuminurie), wat wordt gezien als teken van nierschade. We hebben aangetoond dat patiënten met macroalbuminurie (albumineuitscheiding in de urine $>300 \mathrm{mg} / 24$ uur) significant hogere concentraties van MMP-2, -3 , -10 en TIMP-1 hadden vergeleken met patiënten met een normale albumineuitscheiding in de urine $(<30 \mathrm{mg} / 24$ uur, normoalbuminurie). Patiënten met microalbuminurie (albumine-uitscheiding in de urine tussen de 30 en 300 mg per 24 uur) hadden alleen significante hogere concentraties van MMP-2 vergeleken met patiënten met normoalbuminurie. Concentraties van MMP-2, -3, -10 en TIMP-1 toonden significante trends over de diverse gradaties van albuminurie: normo-, micro- en macroalbuminurie. Vergelijkbare associaties hebben we ook gevonden bij analyses in andere groepen met patiënten met diabetes mellitus type 1.

We hebben ook een positieve associatie gevonden tussen MMP-2 en proliferatieve retinopathie, een gradatie van problemen van de bloedvaten in de ogen. Dit betreft ernstige afwijkingen aan de bloedvaten van de ogen en de non-proliferatieve retinopathie is een minder ernstige variant hiervan, welke ook wel degelijk problemen kan veroorzaken. Geen van de markers was significant verschillend tussen individuen met non-proliferatieve retinopathie en individuen zonder retinopathie. De associatie tussen MMP-2 en proliferatieve retinopathie is vaker onderzocht en geobserveerd bij individuen met diabetes mellitus type 1, maar niet in een studie van deze grootte.

\section{MMP's, TIMP-1 en incidente HVZ en dood}

In hoofdstuk drie hebben we associaties onderzocht tussen circulerende MMP's en TIMP-1 en (niet-)fatale incidente HVZ en dood ongeacht de oorzaak. Hiervoor hebben we gebruik gemaakt van data van een Deense studie met 337 individuen met diabetes mellitus type 1 (LEACE) met normoalbuminurie $(n=167)$ of macroalbuminurie $(n=170)$ 
met een mediane follow-up van 12,3 jaar. Incidente HVZ waren geassocieerd met een hogere circulerende concentratie van MMP-2 op baseline. Hogere baseline concentraties van MMP-1, -2 en -3 waren significant geassocieerd met dood, ongeacht de oorzaak, gedurende de follow-up van ruim 12 jaar.

De significante associatie tussen MMP-2 en incidente HVZ evenals de associaties tussen MMP-3 en dood verdween na correctie voor nierfunctie. Daarbij waren baseline MMP-3 concentraties significant geassocieerd met vermindering van de nierfunctie gedurende follow-up, maar concentraties van MMP-2 op baseline waren dat niet. Dit geeft aan dat de relatie tussen MMP-2/-3 en de uitkomsten beïnvloed wordt door nierfunctie, maar hoe dit precies verloopt kunnen we hier niet uit herleiden.

Pathofysiologisch is MMP-2 geassocieerd met plaqueruptuur, trombusformatie en vasoconstrictie. Daarbij is MMP-2 geassocieerd met toename van vasculaire fibrose en vasculaire gladde spiercelmigratie, maar ook met afname van elastine, een elastische component, in de vaatwand. Hierdoor worden de bloedvaten stijver en tevens minder elastisch. Door deze veranderingen kunnen we de associaties tussen MMP-2 en de hiervoor genoemde uitkomstmaten verklaren. De mogelijke manieren waarop MMP-1 en MMP-3 kunnen bijdragen zijn minder duidelijk.

\section{MMP's, TIMP-1 en arteriële stijfheid}

Toegenomen arteriële stijfheid, het stijver worden van de bloedvaten, is een risicofactor voor het krijgen van HVZ in patiënten met diabetes mellitus type 1. In hoofdstuk vier hebben we gekeken naar cross-sectionele associaties tussen circulerende MMP's en TIMP-1 en twee maten van arteriële stijfheid, te weten: carotis-femoralis pulse wave velocity (cfPWV) en polsdruk. Met cfPWV bepalen we de snelheid van het bloed tussen een bloedvat naar de hersenen (arteria carotis) en een bloedvat in de lies (arteria femoralis). Hoe sneller het bloed zich van het ene naar andere punt verplaatst, hoe stijver het bloedvat. Polsdruk, gedefinieerd als systolische bloeddruk minus diastolisch bloeddruk, is een andere maat voor arteriële stijfheid.

Allereerst hebben we aangetoond dat MMP-3 positief geassocieerd is met cfPWV $(n=614)$, de huidige gouden standaard voor het meten van arteriële stijfheid. De andere markers waren niet significant geassocieerd met cfPWV.

We hebben polsdrukdata van drie verschillende studies met patiënten met diabetes mellitus type 1 en de MMP/TIMP-1-bepalingen ( $n=1517)$. MMP-1 was invers geassocieerd met polsdruk en MMP-2 was positief geassocieerd met polsdruk.

MMP-1 is eerder geassocieerd met verandering in de thoracale aorta leidend tot uitzetting en eventuele aneurysmavorming, welke kan leiden tot een daling van de polsdruk. MMP-2 en MMP-3 waren positief geassocieerd met maten van arteriële stijfheid en dit kan worden verklaard door eerdergenoemde pathofysiologische veranderingen door MMP-2, waarbij MMP-3 grotendeels hetzelfde effect heeft, alsmede het kunnen vrijprepareren van weefselgebonden transforming growth factor-beta 
(TGF- $\beta$ ) door zowel MMP-2 als MMP-3. Dit alles kan leiden tot toename van matrix productie en zodoende vasculaire stijfheid.

\section{Advanced glycation endproducts en het MMP-TIMP systeem}

De vraag blijft wat de primaire factoren zijn die kunnen leiden tot veranderingen in het MMP-TIMP systeem in individuen met diabetes mellitus type 1 . In de algemene introductie staat figuur 1.2, waarin bepaalde bekende pathways naar veranderde ECM worden weergegeven. In de voorgaande hoofdstukken hebben we niet naar losse factoren gekeken die de expressie van MMP's danwel TIMP-1 beïnvloeden. In hoofdstuk vijf hebben we gekeken naar associaties tussen circulerende AGE's en de eerdergenoemde markers van het MMP-TIMP systeem. In de afgelopen jaren zijn uitgebreide associaties tussen AGE's en vasculaire complicaties in diabetes mellitus type 1 beschreven. Daarbij hebben andere studies aangetoond dat blootstelling van cellen aan AGE's leidde tot een toename van de productie van diverse MMP's en TIMP1. Zodoende zouden AGE's een bijdrage kunnen leveren aan veranderingen in de productie van MMP's en/of TIMP-1.

Wij hebben specifiek gekeken naar de eiwitgebonden $A G E^{\prime} s ; N^{\varepsilon}$-(carboxymethyl)lysine $(\mathrm{CML}), \quad \mathrm{N}^{\varepsilon}$-(carboxyethyl)lysine (CEL), 5-hydro-5-methylimidazolone (MG-H1) en pentosidine. In onze cross-sectionele analyses hebben we laten zien dat circulerend $C M L$, een ligand voor de receptoren voor AGE's, positief was geassocieerd met MMP-2 en invers met MMP-9. CEL, een intracellulaire AGE, was positief geassocieerd met MMP-3 en TIMP-1. MG-H1, een ander intracellulaire AGE, was ook geassocieerd met TIMP-1. Pentosidine, een crosslinking AGE, was niet geassocieerd met een van onze markers van het MMP-TIMP system. Deze resultaten ondersteunen grotendeels de conclusies uit eerdere, met name in-vitro, onderzoeken en geeft aanwijzingen dat AGE's mogelijk via MMP's zouden kunnen leiden tot vasculaire complicaties bij patiënten met diabetes mellitus type 1 .

\section{Conclusie}

In dit proefschrift hebben we laten zien dat diverse circulerende markers van het MMPTIMP systeem (MMP-1, -2, -3, en -10 en TIMP-1) geassocieerd zijn, cross-sectioneel danwel prospectief, met diverse vasculaire complicaties en diverse maten van arteriële stijfheid in patiënten met diabetes mellitus type 1. Dit toont aan dat ECM remodelering deel van het pathofysiologisch mechanisme van vasculaire complicaties uitmaakt en dit is nog niet eerder op deze manier aangetoond. Daarnaast hebben we associaties beschreven tussen AGE's en MMP's/TIMP-1. De MMP's en TIMP-1 kunnen dus mogelijk doelen zijn voor preventie en/of behandeling van vasculaire complicaties bij patiënten met diabetes mellitus type 1 . 
Valorization 



\section{Valorization}

As the burden of diabetes mellitus and its complications is increasing worldwide, research regarding the partly unresolved pathophysiological mechanism leading to vascular complications is needed. To date, vascular remodeling is often referred to in case of vascular complications. However, thorough investigations studying extracellular matrix (ECM) remodeling by matrix metalloproteinases (MMPs) and tissue inhibitors of metalloproteinases (TIMPs) in humans with type 1 diabetes have been scarcely published and are lacking extensive adjustments for potential confounders and/or have a small sample size. This thesis focussed on individuals with type 1 diabetes and we showed various associations between circulating MMPs and TIMP-1, as markers of ECM remodeling, and a variety of vascular complications (prior or incident cardiovascular disease (CVD), all-cause mortality, albuminuria, retinopathy, carotid-femoral pulse wave velocity and pulse pressure). In addition, we showed several associations between advanced glycation endproducts (AGEs) and MMPs and/or TIMP-1. However, these findings are primarily epidemiologically and mechanistically, but did not evaluate, for example, social and economical aspects. In this valorization we want to highlight our thoughts on social, economical and clinical issues as this chapter is intended to transfer scientific knowledge into practice.

\section{Social and economical implications}

As the number of individuals with type 1 diabetes is increasing, ${ }^{1}$ decreased quality of life for more individuals worldwide is observed, because type 1 diabetes is chronic disease. Quality of life can even further decrease as patients can become invalidated by its complications. ${ }^{2}$ In addition, complications lead to a rise in costs. Many interventions to prevent and/or control diabetes (type 1 and type 2) have shown to be cost saving or cost-effective. ${ }^{3}$ Therefore, increased knowledge on this subject can lead to several advantages for society. Firstly, with current treatment options we are insufficiently able to prevent or reduce vascular complications in type 1 diabetes. Therefore, this thesis potentially provides additional therapeutical targets and may eventually contribute to the development of new drugs by the pharmaceutical industry. In addition, the use of certain current treatment options can perhaps be started in an earlier stage of the disease to intervene in the MMP-TIMP system, ${ }^{4}$ for example: more intensive insulin treatment and use of ACE-inhibitors and/or statins. Secondly, prevention of these complications may result in increased quality of life and may lead to prolonged lives, as these complications are associated with increased mortality.

Thirdly, prevention of complications can lead to a reduction of, for example, workplace absenteeism, hospital admissions and medicine use. In total, this could lead to an increase in quality of life of patients and a significant decrease in costs for society. 


\section{Clinical implications}

Our research focussed only on individuals with type 1 diabetes (5-10\% of the total diabetic population) and in-hospital diabetic specialists treat these patients. As mentioned previously, perhaps we can intervene earlier with current treatment regimens to prevent early ECM remodeling. However, this option needs to be further investigated.

We do not know if and to what extent the MMP-TIMP system acts similarly in type 2 diabetes. If so, more diabetic individuals worldwide could benefit from our findings. In the Netherlands, type 1 diabetic individuals are treated in hospital, whereas general practitioners mainly treat individuals with type 2 diabetes. In case of (largely) similar actions of the MMP-TIMP system in the two types of diabetes also the general practitioners could benefit from our research.

\section{Future perspectives}

To date, most observed associations, including ours, were cross-sectional and additional (longitudinal) research should be performed to reveal the exact pathophysiological mechanism underlying vascular complications and therefore our research could provide a starting point. Several new questions have also arisen following this thesis:

- Can one of these markers of the MMP-TIMP truly become a therapeutical target to prevent or reduce vascular complications?

- Are our current treatment options (e.g. statins and/or ACE-inhibitors) suitable for prevention or do new pharmaceuticals have to be developed?

- Does the MMP-TIMP system act similarly with regard to the mechanism leading to vascular complications in individuals with type 2 diabetes?

We hope that this thesis and future research will help us to prevent or reduce vascular complications in individuals with type 1 diabetes as well as to prevent additional costs for society. 


\section{References}

1. IDF Diabetes Altas Seventh Edition, International Diabetes Federation 2015.

2. Ducat L, Rubenstein A, Philipson LH, Anderson BJ: A review of the mental health issues of diabetes conference. Diabetes Care 2015;38:333-338

3. Li R, Zhang P, Barker LE, Chowdhury FM, Zhang X: Cost-effectiveness of interventions to prevent and control diabetes mellitus: a systematic review. Diabetes Care 2010;33:1872-1894

4. Chistiakov DA, Sobenin IA, Orekhov AN: Vascular extracellular matrix in atherosclerosis. Cardiol Rev 2013;21:270-288 

Dankwoord 



\section{Dankwoord}

Dit proefschrift is uiteraard het werk van velen en zodoende wil ik in dit dankwoord een aantal mensen in het bijzonder bedanken.

Allereerst wil ik mijn promotieteam bedanken: mijn promotoren prof. dr. C.D.A. Stehouwer en prof. dr. C.G. Schalkwijk en copromotoren dr. L. Engelen en dr. J. Buijs.

Prof. dr. C.D.A. Stehouwer, beste Coen, hartelijk dank dat je mij bij deze wetenschappelijke zoektocht wilde begeleiden. Jouw continue motivatie en drang naar nieuwe inzichten is ongelooflijk. Daarbij ben ik ook nog steeds verbaasd over de specifieke vragen die je bedenkt terwijl ik je tijden niet had gesproken over het onderwerp. Dit heeft mij veel nieuwe zaken geleerd en veel nieuwe inzichten bijgebracht, waarvan ik niet had gedacht dat ik daar ooit over zou nadenken. Sommige zaken hebben even nodig gehad om te bezinken en dank voor jouw geduld daarbij. Ik hoop dat jouw motivatie altijd zal blijven, zowel voor jezelf als voor de onderzoekers die je begeleidt, maar ook voor de patiënten die we uiteindelijk willen helpen door de nieuwe inzichten die we (hopen te) genereren.

Prof. dr. C.G. Schalkwijk, beste Casper, dank voor het goede thuis dat je mij bood op jouw afdeling. Ik heb me erg thuis gevoeld en ben blij dat jouw deur altijd open stond voor een korte vraag of voor een uitgebreide kritische blik op een manuscript. Jouw toegankelijkheid i.c.m. jouw uitgebreide kennis is jouw grote kracht. Hopelijk zullen nog veel promovendi en andere medewerkers van het laboratorium mogen genieten van jouw houding.

Dr. L. Engelen, beste Lian, dank voor jouw (engelen)geduld. Jouw epidemiologische kennis en streven naar perfectie zijn een voorbeeld voor mij geworden. In het begin zul je vaak hebben gedacht: hoe gaat dit ooit goed komen? Ik had geen enkele ervaring met epidemiologie of zelf onderzoek doen en wilde dan toch proberen in een tweejarig traject te promoveren. Je hebt me zeer goed begeleid en jouw toegankelijkheid heeft er ook toe geleid dat we snel zaken konden bespreken. Zodoende zijn we in de eerste twee jaar redelijk gevorderd. Ik ben blij dat je, na jouw vertrek bij de universiteit, toch betrokken wilde blijven bij dit traject.

Dr. J. Buijs, beste Jacqueline, dank voor het (mede) mogelijk maken van dit traject. Als mijn opleider interne geneeskunde in Heerlen hebben we vaker over onderzoek gesproken, maar je hebt dit traject daadwerkelijk mogelijk gemaakt met hulp van het Atrium MC (tegenwoordig Zuyderland). Ik heb veel van je geleerd op zowel medisch gebied als ook op het vlak van onderzoek. Ik hoop dat dit tweejarige traject, waarbij ik 
trots ben om de eerste te zijn geweest, nog vele opvolgers zal krijgen en dat je naast het opleiderschap voor interne geneeskunde ook betrokken zal blijven bij deze passie.

Dit onderzoekstraject is mede mogelijk gemaakt door de leden van de maatschap interne geneeskunde en MDL, de Raad van Bestuur en andere betrokkenen van het voormalige Atrium MC te Heerlen en daarom wil ik hen ook allen danken.

Uiteraard wordt een promotie niet zomaar tot een einde gebracht. Nadat het manuscript klaar was, heeft een vijftal betrokken wetenschappers zich beraden op de inhoud: de leescommissie. Hierbij wil ik prof. dr. N.C. Schaper (voorzitter), dr. ir. K.D. Reesink, prof. dr. A.J. Smit, dr. S.S. Soedamah-Muthu en prof. dr. M.P. Weijenberg bedanken voor hun bereidheid om dit proefschrift beoordelen.

As I mentioned in the first sentence: this is work of many others. Hereby I would like to thank the co-autors (N. Chaturvedi, J.H. Fuller, A. Jorsal, L. Tarnow, H.-H. Parving, S. Theilade and P. Rossing), who contributed significantly to the manuscripts.

De eerlijkheid gebied te zeggen dat ikzelf niets heb gedaan voor de bepalingen van de circulerende concentraties van de MMP's en TIMP-1. Drs. M. van de Waarenburg, beste Marjo, hartelijk dank voor de bepalingen van de concentraties van de enzymen. Je hebt in ruim 1500 patiënten een vijftal MMP's en TIMP-1 bepaald. Dan heb ik het nog niet gehad over de 8 weken die je nodig had om met Jean de AGE's te bepalen voor het laatste manuscript. Daarnaast wil ik je ook danken voor de gezelligheid die we hebben gehad zowel op de werkvloer als bij de vele koffiemomenten.

Dr. J.L.J.M. Scheijen, beste Jean, tegen de tijd dat dit boekje verschijnt, ben je reeds zelf gepromoveerd. Nogmaals gefeliciteerd! Ik wil ook jou bedanken zowel op professioneel als persoonlijk vlak. Je hebt met Marjo vele uren (lees: weken) besteed aan de bepalingen van de AGE's. Daarnaast hebben we vele mooie momenten meegemaakt en dan heb ik het met name over de stapavonden tot in de vroege uurtjes in zowel binnenals buitenland. Onze avond in Wenen was inderdaad legendarisch, maar kan zeker worden overtroffen en dat zullen we nog zeker gaan waarmaken! Ik wens je veel succes toe bij jouw toekomstige werkzaamheden en publicaties.

Lieve Monica en Ellis, ik ben blij dat jullie mijn paranimfen willen zijn! Monica, we hebben elkaar ontmoet op mijn eerste werkdag in het Atrium MC op afdeling 8-West, waar je toen nog semi-arts was. Ellis, onze eerste ontmoeting was bij jouw sollicitatie voor een ANIOS plek niet veel later. We doorlopen nu allen een iets andere route, maar met het gemeenschappelijke doel om uiteindelijk een gepromoveerde internist te worden en dat gaan we allemaal redden! Ik ben nu de eerste die zijn boekje mag verdedigen, maar gelukkig zullen jullie ook spoedig volgen. We hebben veel gesproken 
over het werk en regelmatig over de zaken waar we tegenaan liepen, maar het voornaamste is wel dat we veel leuke dingen hebben meegemaakt. Van de koffiemomenten op de universiteit tot borrels diep in de nacht bij congressen in het buitenland of in een lokale Belgische discotheek. Werk is leuk en belangrijk, maar ik vind dit belangrijker en we gaan waarschijnlijk nog veel leuke etentjes, borrels en congressen meemaken. Veel succes met de afronding van jullie boekjes en het vervolg in de kliniek.

Naast eerdergenoemden heb ik natuurlijk met nog veel meer mensen samengewerkt en zeer leuke tijden beleefd. Allereerst wil ik de collega's van de interne geneeskunde/MDL in het Zuyderland ziekenhuis in Heerlen bedanken voor de leuke tijd en de interesse in mijn traject. Daarnaast heb ik veel lol beleefd met collega's op de universiteit (in alfabetische volgorde): Ankie, Ben, Boy, Dennis, Dionne, Elisabeth, Hanneke, José, Katrien, Kristiaan, Marcelle, Margee, Maria, Marleen, Marnix, Mathias, Mitchell, Nordin, Nynke, Olaf, Pauline, Petra, Suzan, Thomas, Vicky en Yvo. Ook was er veel interesse van de collega's in Maastricht UMC+, waarvoor veel dank en dank voor het kunnen sparren over de laatste loodjes van dit proefschrift.

Natuurlijk mogen ook mijn vrienden en (schoon)familie niet vergeten worden. Ondanks dat we een aantal jaar geleden naar het diepe zuiden zijn verhuisd, is het fijn om met veel van jullie nog goed contact te hebben. Ik hoop dat er bij het einde van dit traject wat meer tijd vrij komt om af te spreken.

Beste Sander en Suzy, dank voor jullie interesse en, Sander, misschien kan dit proefschrift een aanleiding vormen om ook te gaan promoveren, want zoals pappa altijd zei: je moet de 's' van sukkel nog maar eens zien kwijt te raken.

Lieve mam, jij en pappa hebben ons altijd vrij gelaten om bepaalde keuzes te maken, maar ook gestimuleerd om het beste uit alles te halen. Zodoende heb ik ook besloten om mijn VWO-examen opnieuw te doen om hogere cijfers te halen en aansluitend geneeskunde te gaan studeren. Gelukkig heeft pappa mijn afstuderen nog meegemaakt, maar dit moment helaas niet. Voor sommige (langdurige) projecten (lees: geneeskunde, specialisatie en promotie) heb ik inspirerende personen nodig en jullie zijn voor mij deze voorbeelden.

Lieve Manon, we zijn nu ruim 16 jaar samen. We kunnen elkaars gedachten lezen en zijn veel samen, maar kunnen elkaar ook vrij laten en dat zorgt ervoor dat we een goed koppel/team zijn. Ons team is reeds uitgebreid met Floris en binnenkort wordt nummer twee verwacht. Ik ben blij met jouw geduld en dankbaar voor de vele weekenden die je grotendeels alleen met Floris hebt doorgebracht, omdat ik weer aan het werk was. Wij zijn en blijven een fantastisch team, voor altijd! 

Curriculum vitae 



\section{Curriculum vitae}

Stijn Armand Peeters was born on February $14^{\text {th }} 1983$ in Tilburg. He graduated from secondary school twice at Luzac College Breda (VWO, 2001 and 2002). In 2002 he started medical school at Radboud University in Nijmegen, the Netherlands. He obtained his medical degree in 2010 and worked for six months at the department of Internal Medicine at Canisius Wilhelmina Hospital in Nijmegen. On December $1^{\text {st }} 2010$ he started his specialisation in Internal Medicine at the department of Internal Medicine at Atrium Medical Centre in Heerlen, the Netherlands. From July 2013 until July 2015 he spent two years at the research department of Internal Medicine at Maastricht University in which he started his PhD research. After this period he continued his residency in Atrium Medical Centre/Zuyderland. On December $1^{\text {st }} 2016$ he started at the department of Endocrinology in Maastricht University Medical Centre+ in Maastricht, the Netherlands. 

Scientific output 



\section{Scientific output}

\section{List of publications}

J. Aalten, S.A. Peeters, M.J. van der Vlugt, A.J. Hoitsma

Is standardized cardiac assessment of asymptomatic high-risk renal transplant candidates beneficial?

Nephrol Dial Transplant. 2011 Sep;26(9):3006

\section{S.A. Peeters, R. Bianchi, J. Buijs}

Insuline gecombineerd met een DPP-4-remmer: een nieuwe behandeloptie bij type 2 diabetes mellitus

Ned Tijdschr Diabet. 2014;12:104

S.A. Peeters, L. Engelen, J. Buijs, N. Chaturvedi, J.H. Fuller, C.G. Schalkwijk, C.D.A. Stehouwer; EURODIAB Prospective Complications Study Group

Plasma levels of matrix metalloproteinase-2, -3, -10, and tissue inhibitor of metalloproteinase-1 are associated with vascular complications in patients with type 1 diabetes. The EURODIAB Prospective Complications Study

Cardiovasc Diabetol. 2015 Mar 10;14:31

S.A. Peeters, L. Engelen, J. Buijs, N. Chaturvedi, J.H. Fuller, C.G. Schalkwijk, C.D.A. Stehouwer; EURODIAB Prospective Complications Study Group

Erratum to: Plasma levels of matrix metalloproteinase-2, -3, -10, and tissue inhibitor of metalloproteinase-1 are associated with vascular complications in patients with type 1 diabetes. The EURODIAB Prospective Complications Study

Cardiovasc Diabetol. 2015 Sep 28;14:128

S.A. Peeters, L. Engelen, J. Buijs, A. Jorsal, H.-H. Parving, L. Tarnow, P. Rossing, C.G. Schalkwijk, C.D.A. Stehouwer

Plasma matrix metalloproteinases are associated with incident cardiovascular disease and all-cause mortality in patients with type 1 diabetes: a 12-yr follow-up study

Cardiovasc Diabetol. 2017 Apr 26;16(1):55

S.A. Peeters, L. Engelen, J. Buijs, N. Chaturvedi, J.H. Fuller, A. Jorsal, H.-H. Parving, L. Tarnow, S. Theilade, P. Rossing, C.G. Schalkwijk, C.D.A. Stehouwer

Circulating matrix metalloproteinases are associated with arterial stiffness in patients with type 1 diabetes: pooled analysis of three cohort studies

Cardiovasc Diabetol. Accepted for publication 


\section{Submitted manuscript}

S.A. Peeters, L. Engelen, J. Buijs, S. Theilade, P. Rossing, C.G. Schalkwijk, C.D.A. Stehouwer Associations between advanced glycation endproducts and matrix metalloproteinases and its inhibitor in individuals with type 1 diabetes

\section{Oral presentations}

\section{EASD 2014}

Plasma levels of matrix metalloproteinase-2, -3, -10, and tissue inhibitor of metalloproteinase-1 are associated with vascular complications in patients with type 1 diabetes. The EURODIAB Prospective Complications Study

\section{EDNSG 2015}

Plasma levels of matrix metalloproteinase-2, -3, -10, and tissue inhibitor of metalloproteinase-1 are associated with vascular complications in patients with type 1 diabetes. The EURODIAB Prospective Complications Study

Plasma matrix metalloproteinases are associated with incident cardiovascular disease and all-cause mortality in patients with type 1 diabetes: a 12-yr follow-up study

\section{Poster presentation}

EASD 2017

Plasma matrix metalloproteinases are associated with incident cardiovascular disease and all-cause mortality in patients with type 1 diabetes: a 12-yr follow-up study 
Abbreviations 



\section{Abbreviations}

\begin{tabular}{|c|c|}
\hline ABPM & Ambulatory blood pressure measurements \\
\hline ACE & Angiotensin converting enzyme \\
\hline AGES & Advanced glycation endproducts \\
\hline$\beta$-cells & Beta-cells \\
\hline baPWV & Brachial ankle pulse wave velocity \\
\hline $\mathrm{BMI}$ & Body mass index \\
\hline CEL & $\mathrm{N}^{\varepsilon}$-(carboxyethyl)lysine \\
\hline cfPWV & Carotid-femoral pulse wave velocity \\
\hline CKD-EPI & Chronic Kidney Disease Epidemiology Collaboration \\
\hline CML & $\mathrm{N}^{\varepsilon}$-(carboxymethyl)lysine \\
\hline CRP & C-reactive protein \\
\hline CTGF & Connective tissue growth factor \\
\hline CVD & Cardiovascular disease \\
\hline ECM & Extracellular matrix \\
\hline ED & Endothelial dysfunction \\
\hline eGFR & Estimated glomerular filtration rate \\
\hline ELISA & Enzyme-linked immunosorbent assay \\
\hline GAD65 & Glutamic acid decarboxylase \\
\hline $\mathrm{HbA1c}$ & Haemoglobin A1c \\
\hline HDL & High-density lipoprotein \\
\hline $\mathrm{HR}$ & Hazard ratio \\
\hline $\mid \mathrm{A}$ & Insulinoma antigen \\
\hline IC & Islet cells \\
\hline ICAM & Intercellular adhesion molecule \\
\hline IL-6 & Interleukin-6 \\
\hline LADA & Latent autoimmune diabetes of the adult \\
\hline LDL & Low-density lipoprotein \\
\hline LGI & Low-grade inflammation \\
\hline MAP & Mean arterial pressure \\
\hline MCP-3 & Monocyte chemo-attractant protein-3 \\
\hline MG-H1 & 5-hydro-5-methylimidazolone \\
\hline MGO & Methylglyoxal \\
\hline MMP & Matrix metalloproteinase \\
\hline MODY & Maturity-onset diabetes of the young \\
\hline PAl-1 & Plasminogeen activator inhibitor-1 \\
\hline PLA2 & Phospholipase A2 \\
\hline PP & Pulse pressure \\
\hline RAAS & Renin angiotensin aldosterone system \\
\hline RAGE & Receptor of advanced glycation endproduct \\
\hline
\end{tabular}



Tissue inhibitor of metalloproteinase

TNF- $\alpha$ Tumor necrosis factor-alfa

UAE Urinary albumin excretion

UAER Urinary albumin excretion ratio

VCAM Vascular cell adhesion molecule

VSMC Vascular smooth muscle cell 